\title{
Clinical psychological and economic aspects of pregnancy care in women with a history of preeclampsia or HELP syndrome
}

Citation for published version (APA):

van de Venne-Delahaije, D. H. L. (2014). Clinical psychological and economic aspects of pregnancy care in women with a history of preeclampsia or HELP syndrome. [Doctoral Thesis, Maastricht University]. Datawyse / Universitaire Pers Maastricht. https://doi.org/10.26481/dis.20141127dv

Document status and date:

Published: 01/01/2014

DOI:

10.26481/dis.20141127dv

Document Version:

Publisher's PDF, also known as Version of record

Please check the document version of this publication:

- A submitted manuscript is the version of the article upon submission and before peer-review. There can be important differences between the submitted version and the official published version of record.

People interested in the research are advised to contact the author for the final version of the publication, or visit the DOI to the publisher's website.

- The final author version and the galley proof are versions of the publication after peer review.

- The final published version features the final layout of the paper including the volume, issue and page numbers.

Link to publication

\footnotetext{
General rights rights.

- You may freely distribute the URL identifying the publication in the public portal. please follow below link for the End User Agreement:

www.umlib.nl/taverne-license

Take down policy

If you believe that this document breaches copyright please contact us at:

repository@maastrichtuniversity.nl

providing details and we will investigate your claim.
}

Copyright and moral rights for the publications made accessible in the public portal are retained by the authors and/or other copyright owners and it is a condition of accessing publications that users recognise and abide by the legal requirements associated with these

- Users may download and print one copy of any publication from the public portal for the purpose of private study or research.

- You may not further distribute the material or use it for any profit-making activity or commercial gain

If the publication is distributed under the terms of Article $25 \mathrm{fa}$ of the Dutch Copyright Act, indicated by the "Taverne" license above, 
Denise HJ van de Venne-Delahaije

Clinical, psychological and economic aspects of pregnancy care in women with a history of preeclampsia or HELLP syndrome 
The research described in this thesis was funded by the Netherlands Organisation for Health Research and Development (ZonMw grant nr. 170882303).

Financial support for printing of this thesis was kindly provided by Stichting HELLP-syndroom

Postbus 636

3800 AP Amersfoort

ISBN 978-94-6159-357-3

Copyright (C) 2014, D.H.J. van de Venne-Delahaije, Melick

Cover and design: Datawyse

Printed by: Datawyse / Universitaire Pers Maastricht 


\title{
Clinical, psychological and economic aspects of pregnancy care in women with a history of preeclampsia or HELLP syndrome
}

\author{
PROEFSCHRIFT \\ ter verkrijging van de graad van doctor aan de Universiteit Maastricht, \\ op gezag van de Rector Magnificus, Prof. dr. L.L.G. Soete, \\ volgens het besluit van het College van Decanen, \\ in het openbaar te verdedigen \\ op donderdag 27 november 2014 om 14.00 uur \\ door \\ Denise Hubertine Josephine van de Venne-Delahaije \\ Geboren op 16 maart 1984 te Heerlen
}

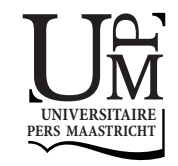




\section{Promotor}

Prof. dr. C.D. Dirksen

\section{Copromotores}

Dr. L.J.M. Smits

Dr. L.L.H. Peeters (UMC Utrecht)

\section{Beoordelingscommissie}

Prof. dr. S.M.A.A. Evers (voorzitter)

Prof. dr. I.M. Engelhard (Universiteit Utrecht)

Dr. A. Kwee (UMC Utrecht)

Prof. dr. J.G. Nijhuis

Prof. dr. M.H. Prins 


\section{CONTENTS}

$\begin{array}{lll}\text { Chapter } 1 \text { General introduction } & 7\end{array}$

Chapter 2 Cost-effectiveness of recurrence risk guided care versus care-

as-usual in women who suffered from early-onset preeclampsia

including HELLP syndrome in their previous pregnancy (the

PreCare study)

Chapter 3 Care-as-usual provided to formerly preeclamptic women in the

Netherlands in their next pregnancy: health care consumption, costs and pregnancy outcome

Chapter 4 Cost-effectiveness of recurrence risk guided care in the next pregnancy of women who experienced early-onset preeclampsia or HELLP syndrome in their previous pregnancy

Chapter 5 Patient satisfaction with recurrence risk guided care in pregnant women with previous preeclampsia or HELLP syndrome

Chapter 6 Anxiety and depression following preeclampsia or HELLP

syndrome. A systematic review

Chapter 7 Summary and General discussion

Chapter 8 Nederlandstalige samenvatting

Valorization

Dankwoord

Curriculum vitae 

CHAPTER 1

General introduction

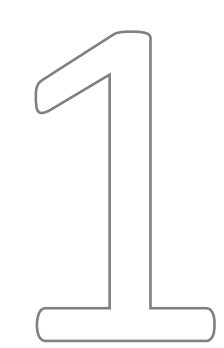




\section{Preeclampsia and HELLP syndrome}

Preeclampsia (PE) is defined as the de-novo development of hypertension ( $\geq 140 / 90$ $\mathrm{mmHg}$ ) along with the de-novo proteinuria ( $\geq 300 \mathrm{mg} /$ day proteinuria) after the $20^{\text {th }}$ week of pregnancy [1]. The HELLP syndrome, a more severe form of this disorder, is defined on the basis of the acronyms of its symptoms (ㅂemolysis, Elevated Liver enzymes and Low Platelets). The prevalence of PE is 2-5\% of first pregnancies, with about $10 \%$ becoming complicated by the HELLP syndrome [2, 3]. PE and HELLP are a serious pregnancy complication as indicated by the associated high maternal and perinatal morbidity and mortality. In the developed world, 10-25\% of all pregnancy-related maternal deaths are attributed to PE or HELLP [4]. About $1 \%$ of women with PE and HELLP deteriorate to eclampsia, a severe complication characterized by generalized convulsions most likely triggered by cerebral edema and vasospasm. After delivery, the typical clinical signs of PE and HELLP disappear in most cases within 48 hours. Perinatal mortality in $\mathrm{PE}$ is high (10\%) and related to the central role of placental dysfunction in PE and the HELLP syndrome [5-7].

Although placental dysfunction is generally considered to be the starting point in the clinical manifestation of PE and HELLP, its etiology is still obscure. Most nulliparous women who develop PE were normotensive before developing the hypertensive pregnancy disorder. Nevertheless, pre-pregnant obesity with or without metabolic syndrome or a family history of hypertension and PE are known to be predisposing conditions [8-11]. Because of its enigmatic etiology, the only causal treatment of PE/HELLP is termination of pregnancy.

Box 1: Definitions of hypertensive pregnancy complications used in this thesis

$\begin{array}{ll}\text { Preeclampsia } & \text { The de-novo development of hypertension ( } \geq 140 / 90 \mathrm{mmHg} \text { ) in combination with the de- } \\ & \text { novo development of proteinuria ( } \geq 300 \mathrm{mg} \text { urinary protein excretion/day) after } 20 \text { weeks } \\ & \text { pregnancy }\end{array}$

HELLP syndrome A combination of excessively high turnover rate of red blood cells ( $\mathrm{H}$ : Hemolysis), rise in the circulating levels of liver enzymes secondary to liver cell necrosis (EL: Elevated Liver enzymes), and fall in number of circulating thrombocytes (LP: Low Platelet count, platelets $<100 \times 10^{9} /$ I) developing in pregnancy. HELLP is considered a complication of PE or eclampsia, but may also develop without prior hypertension and/or proteinuria

Eclampsia The development of generalized seizures in pregnancy, almost always in a woman previously diagnosed with pregnancy-induced hypertension, preeclampsia and/or the HELLP syndrome

Early-onset Onset of PE before the $34^{\text {th }}$ week of pregnancy 


\section{Recurrent PE/HELLP, target for prediction and tailored management}

Although PE and HELLP are mostly considered disorders typically occurring in first pregnancies, pregnant women with a history of PE or HELLP are at increased risk of developing pregnancy-induced hypertension, PE, or the HELLP syndrome and/or fetal growth restriction due to placental insufficiency $[13,14]$. Estimates of the recurrence rate vary from 0 to $31 \%$ for PE and from 3 to $7 \%$ for HELLP [15]. If PE or HELLP recurs in the second pregnancy, the clinical course of the recurrence tends to be more serious. This is indicated by a higher rate of early-onset PE. The latter is associated with a higher incidence of preterm pregnancy termination, and with it, more premature births, often accompanied by fetal growth restriction. It follows that these infants require more neonatal intensive care and suffer of more complications [16]. Therefore, current management of pregnant women with a history of early-onset PE entails close and frequent surveillance. Currently, there is no clinical practice guideline for the management of these women. As a consequence, care varies per center, gynecologist and patient and -in the next pregnancy- depends on the perceived recurrence risk, the former patient's anxiety and her demand for care. This opportunistic, informal type of care is referred to as care-as-usual (CAU).

Although a history of PE or HELLP is associated with an increased risk of recurrence, course and outcome of most next pregnancies will be normal. However, gynecologists have neither a sensitive test to predict nor an effective management strategy in high-risk women to prevent or alleviate an early-onset recurrence. Therefore, identifying the larger subgroup of women with a low risk of recurrence among former patients would restrict the burden of intensified surveillance to a relatively small subgroup of former patients, thus lowering the level of medicalization and associated stress in most of former patients while also reducing (health care) costs. Recent trends support personalized medicine, which stands for the use of management tailored to an individual's (risk) profile [17].

\section{Predicting recurrent PE and HELLP}

Although earlier studies have identified several individual predictors for recurrent PE and HELLP, combining biomarkers for this purpose has only been explored recently [18-24]. Based on data collected from former patients, who not only experienced early-onset PE or HELLP in their first pregnancy, but also had a well-documented course and outcome of their next pregnancy, we constructed a prediction model to estimate the recurrence risk in women with a history of PE/HELLP. We selected the following biomarkers in a multivariable logistic regression model: 1) Circulating fasting glucose 
level measured at postpartum screening; 2) Gestational age at birth of the previous complicated pregnancy; 3) Birth of a small-for-gestational-age after that pregnancy; 4) Prepregnancy maternal body mass index, and 5) Chronic hypertension diagnosed prior to the second pregnancy [25]. The model was validated internally. Discrimination of the model, after correction for optimism by bootstrapping, was adequate $(0.65 ; 95 \% \mathrm{Cl}$ : 0.56-0.74). The calibration of the model was good, indicated by a non-significant Hosmer-Lemeshow test $(p=0.11)$.

\section{The PreCare study}

The prediction model which was developed by our research group was expected to help in identifying women at really low risk of recurrent early-onset PE/HELLP. The PreCare study was designed to assess the effect of risk stratification using this prediction model on maternal and neonatal outcome, patient satisfaction and (health care and societal) costs.

Based on the prediction model, women received either protocolized Medium Care (MC) or High Care $(\mathrm{HC})$, depending on their predicted risk of developing early-onset recurrent PE/HELLP (recurrence risk guided care, RGC). The MC and HC protocols contained 9-11 and 14-16 visits to the outpatient clinic, respectively. Women receiving HC could also be offered additional diagnostic tests. We compared RGC with CAU for both pregnancy-related health care and non-health care related costs (e.g. productivity loss, informal care) and generic health-related quality of life and adverse neonatal outcomes.

\section{Economic evaluation in health care}

Nowadays, economic evaluations are becoming increasingly important as resources are finite. Therefore, new guidelines for clinical practice should also consider the impact on health care expenses of evidence-based clinical management recommendations [26]. Economic evaluation in health care refers to the comparison of alternative treatment options in terms of their costs and consequences [27]. Alternative treatment options refer to the range of ways in which health care resources can be used to increase population health, e.g. pharmaceutical and surgical interventions, screening and health promotion programs. Health care costs refer to the value of tangible resources available to the health care system, e.g. buildings, equipment, consumables such as drugs and disposables, and clinical and other staff. Non-health care resources used in health care are e.g. time invested by a patient's family. Consequences repre- 
sent all the effects of health care interventions other than those on resources. These generally focus on changes in an individual's health, which can be positive or negative, but can also include other effects that individuals may value, such as reassurance and proper information provision.

There are several types of economic evaluations that differ by how the consequences of health care interventions are taken into account [27]. A cost-minimization analysis only compares the costs of two interventions, presuming a similar beneficial health effect. A cost-benefit analysis considers both costs and effects in monetary terms. In a cost-effectiveness analysis, the cost-effectiveness ratio is used to compare two intervention options aimed at achieving the same goal. A special type of costeffectiveness analysis is called cost-utility analysis, in which the effect consists of health-related quality of life combined with life expectancy, so-called Quality Adjusted Life Years (QALYs).

\section{Patient satisfaction}

Most economic evaluations fail to adequately incorporate the patient's perspective [28]. However, patient preferences are considered to be increasingly important in health care policy decision-making $[28,29]$. One approach to consider the patient's perspective in research is to investigate patient's satisfaction with the treatment. The concept 'satisfaction' simply captures the patient's point of view about the treatment received. Integrating the patient's perspective in health care decision-making is important for several reasons. Exploring care quality from the patient's perspective can reveal important information as a patient's expectations from or satisfaction about a new treatment may differ from that of the clinical expert [30,31]. The relevance of patient satisfaction is further emphasized by experimental evidence suggesting that user acceptability optimizes the cost-effectiveness of a new type of care (e.g. through protocol adherence), resulting in both better implementation and a higher quality of life in satisfied patients [32-34]. Patients may respond better to treatment and comply better to guidelines when they are satisfied with their care and treatment setting [3537]. Meanwhile, areas for improvement can be identified.

In the PreCare study as elaborated in this dissertation, we did our utmost best to explore whether patients were satisfied with RGC. Therefore, the evaluation of RGC not only consisted of a detailed assessment of (pregnancy) outcome and costs, but also of an evaluation of patient satisfaction. 


\section{Mental disorders in women with previous PE or HELLP}

Women with a history of PE/HELLP are at increased risk of developing posttraumatic stress disorder shortly after discharge from hospital, presumably as a direct consequence of experiencing excessive stress because of giving preterm birth, emergency cesarean section, and maternal and neonatal complications [38]. A better understanding of the incidence and severity of anxiety and depression in former PE or HELLP patients is expected to enable prevention and targeted interventions. Currently, diagnosis and management of anxiety and depression in the symptomatic phase of preeclampsia and the following postpartum period are still suboptimal [12].

\section{Aim and outline of the thesis}

The main objective of this thesis was to compare RGC with CAU in the next pregnancy of women with a previous early-onset preeclamptic pregnancy, with respect to costs, pregnancy outcome, patient satisfaction, and the psychopathologic impact of PE/HELLP. The thesis consists of seven chapters.

Chapter 2 provides a detailed description of the study design. The PreCare study uses a before-after design, comparing costs and pregnancy outcome before (i.e. careas-usual, $\mathrm{CAU}$ ) and after the introduction of the prediction model (i.e. recurrence risk guided care, RGC). Chapter 3 describes the care-as-usual part of the study, including hospital consumption, associated costs and pregnancy outcome. Characterizing CAU is an essential first step in identifying the positive and negative components of care, serving as a starting point for implementing evidence-based practice and identifying potential barriers. Chapter 4 describes the results of the economic evaluation. We calculated two incremental cost-effectiveness ratios (ICERs) consisting of 1) the cost per Quality Adjusted Life Year, i.e. QALY (mother unit of analysis, and 2) the cost per prevented adverse outcome child, i.e. NICU admission and/or death (child unit of analysis). Chapter 5 describes satisfaction rates in RGC subdivided into overall satisfaction, technical quality, interpersonal aspects, communication, time spent with doctor and access/availability/convenience. Based on Crow's holistic model of satisfaction, we performed multivariate regression analyses to explore various contributors to satisfaction and protocol adherence. Chapter 6 comprises a systematic review on whether or not a history of PE/HELLP is associated with anxiety and depression and whether $\mathrm{PE} / \mathrm{HELLP}$ is an independent risk factor for developing anxiety and depression. In the final chapter, chapter 7 , the results of this study are summarized and discussed. 


\section{References}

1. Brown MA, Lindheimer MD, de Swiet M, Van Assche A, Moutquin JM: The classification and diagnosis of the hypertensive disorders of pregnancy: statement from the International Society for the Study of Hypertension in Pregnancy (ISSHP). Hypertension in pregnancy : official journal of the International Society for the Study of Hypertension in Pregnancy 2001, 20(1):IX-XIV.

2. Meads CA, Cnossen JS, Meher S, Juarez-Garcia A, ter Riet G, Duley L, Roberts TE, Mol BW, van der Post $J A$, Leeflang $M M$ et al: Methods of prediction and prevention of pre-eclampsia: systematic reviews of accuracy and effectiveness literature with economic modelling. Health Technol Assess 2008, 12(6):iii-iv, 1-270.

3. Vuori H: Patient satisfaction--does it matter? Quality assurance in health care : the official journal of the International Society for Quality Assurance in Health Care / ISQA 1991, 3(3):183-189.

4. Khan KS, Wojdyla D, Say L, Gulmezoglu AM, Van Look PF: WHO analysis of causes of maternal death: a systematic review. Lancet 2006, 367(9516):1066-1074.

5. Crowhurst JA, Plaat F: Why mothers die--report on confidential enquiries into maternal deaths in the United Kingdom 1994-96. Anaesthesia 1999, 54(3):207-209.

6. Ananth CV, Savitz DA, Bowes WA, Jr.: Hypertensive disorders of pregnancy and stillbirth in North Carolina, 1988 to 1991. Acta obstetricia et gynecologica Scandinavica 1995, 74(10):788-793.

7. van Beeck EF, van Roijen L, Mackenbach JP: Medical costs and economic production losses due to injuries in the Netherlands. The Journal of trauma 1997, 42(6):1116-1123.

8. Reyes LM, Garcia RG, Ruiz SL, Camacho PA, Ospina MB, Aroca G, Accini JL, Lopez-Jaramillo P: Risk factors for preeclampsia in women from Colombia: a case-control study. PloS one 2012, 7(7):e41622.

9. Schneider S, Freerksen N, Rohrig S, Hoeft B, Maul H: Gestational diabetes and preeclampsia--similar risk factor profiles? Early human development 2012, 88(3):179-184.

10. Shamsi U, Hatcher J, Shamsi A, Zuberi N, Qadri Z, Saleem S: A multicentre matched case control study of risk factors for preeclampsia in healthy women in Pakistan. BMC women's health 2010, 10:14.

11. Dalla Palma L, Pozzi Mucelli R, Sponza M, Bartolozzi C, De Santis M, Gandini G, Mannella P, Matricardi L, Rossi C, Simonetti G: [Comparative evaluation of ultrasonography, computerized tomography, angiography and lipiodol CT in defining extent of hepatocarcinoma. A multicenter study]. La Radiologia medica 1995, 89(3):270-277.

12. Kelly R, Zatzick D, Anders T: The detection and treatment of psychiatric disorders and substance use among pregnant women cared for in obstetrics. The American journal of psychiatry 2001, 158(2):213219.

13. Langenveld J, Buttinger A, van der Post J, Wolf H, Mol BW, Ganzevoort W: Recurrence risk and prediction of a delivery under 34 weeks of gestation after a history of a severe hypertensive disorder. BJOG : an international journal of obstetrics and gynaecology 2011, 118(5):589-595.

14. Andersgaard AB, Acharya G, Mathiesen EB, Johnsen SH, Straume B, Oian P: Recurrence and long-term maternal health risks of hypertensive disorders of pregnancy: a population-based study. American journal of obstetrics and gynecology 2012, 206(2):143 e141-148.

15. Sep S, Smits L, Prins M, Peeters L: Prediction tests for recurrent hypertensive disease in pregnancy, a systematic review. Hypertension in pregnancy : official journal of the International Society for the Study of Hypertension in Pregnancy 2010, 29(2):206-230.

16. Hnat MD, Sibai BM, Caritis S, Hauth J, Lindheimer MD, MacPherson C, VanDorsten JP, Landon M, Miodovnik M, Paul R et al: Perinatal outcome in women with recurrent preeclampsia compared with women who develop preeclampsia as nulliparas. American journal of obstetrics and gynecology 2002, 186(3):422-426.

17. Chan IS, Ginsburg GS: Personalized medicine: progress and promise. Annual review of genomics and human genetics 2011, 12:217-244. 
18. Conde-Agudelo A, Villar J, Lindheimer M: World Health Organization systematic review of screening tests for preeclampsia. Obstetrics and gynecology 2004, 104(6):1367-1391.

19. Aardenburg R, Spaanderman ME, Ekhart TH, van Eijndhoven HW, van der Heijden OW, Peeters LL: Low plasma volume following pregnancy complicated by pre-eclampsia predisposes for hypertensive disease in a next pregnancy. BJOG : an international journal of obstetrics and gynaecology 2003, 110(11):10011006.

20. Branch DW, Porter TF, Rittenhouse L, Caritis S, Sibai B, Hogg B, Lindheimer MD, Klebanoff M, MacPherson C, VanDorsten JP et al: Antiphospholipid antibodies in women at risk for preeclampsia. American journal of obstetrics and gynecology 2001, 184(5):825-832; discussion 832-824.

21. Chames MC, Haddad B, Barton JR, Livingston JC, Sibai BM: Subsequent pregnancy outcome in women with a history of HELLP syndrome at < or $=28$ weeks of gestation. American journal of obstetrics and gynecology 2003, 188(6):1504-1507; discussion 1507-1508.

22. Hjartardottir S, Leifsson BG, Geirsson RT, Steinthorsdottir V: Paternity change and the recurrence risk in familial hypertensive disorder in pregnancy. Hypertension in pregnancy : official journal of the International Society for the Study of Hypertension in Pregnancy 2004, 23(2):219-225.

23. Trogstad L, Skrondal A, Stoltenberg C, Magnus P, Nesheim BI, Eskild A: Recurrence risk of preeclampsia in twin and singleton pregnancies. American journal of medical genetics Part A 2004, 126A(1):41-45.

24. Zhang J, Troendle JF, Levine RJ: Risks of hypertensive disorders in the second pregnancy. Paediatric and perinatal epidemiology 2001, 15(3):226-231.

25. van Kuijk SM, Nijdam ME, Janssen KJ, Sep SJ, Peeters LL, Delahaije DH, Spaanderman M, Bruinse HW, Franx A, Bots $\mathrm{ML}$ et al: A model for preconceptional prediction of recurrent early-onset preeclampsia: derivation and internal validation. Reprod Sci 2011, 18(11):1154-1159.

26. Petitti DB: Meta-analysis, decision analysis and cost-effectiveness analysis: Methods for quantitative synthesis in medicine., Second edition edn. New York: Oxford University Press; 2000.

27. Drummond MF, Sculpher MJ, Torrance GW, B.J. OB, Stoddart GL: Methods for the economic evaluation of health care programmes, 3rd edition edn. Oxford: Oxford University Press; 2005.

28. Bridges JF, Jones C: Patient-based health technology assessment: a vision of the future. International journal of technology assessment in health care 2007, 23(1):30-35.

29. Dirksen CD, Utens CMA, Joore MA, van Barneveld TA, Boer B, Dreesens DHH, van Laarhoven $H$, Smit $C$, Stiggelbout AM, van der Weijden T: Integrating evidence on patient preferences in healthcare policy decisions: protocol of the patient-VIP study. Implementing Science 2013, 8(64).

30. Merkel WT: Physician perception of patient satisfaction. Do doctors know which patients are satisfied? Medical care 1984, 22(5):453-459.

31. Street RL, Haidet P: How well do doctors know their patients? Factors affecting physician understanding of patients' health beliefs. J Gen Intern Med 2011, 26(1):21-27.

32. Crow R, Gage H, Hampson S, Hart J, Kimber A, Storey L, Thomas H: The measurement of satisfaction with healthcare: implications for practice from a systematic review of the literature. Health Technol Assess 2002, 6(32):1-244.

33. Safran DG, Taira DA, Rogers WH, Kosinski M, Ware JE, Tarlov AR: Linking primary care performance to outcomes of care. The Journal of family practice 1998, 47(3):213-220.

34. Guldvog B: Can patient satisfaction improve health among patients with angina pectoris? Int J Qual Health Care 1999, 11(3):233-240.

35. Jackson JL, Chamberlin J, Kroenke K: Predictors of patient satisfaction. Soc Sci Med 2001, 52(4):609-620.

36. Kizer KW: Patient centred care: essential but probably not sufficient. Quality \& safety in health care 2002, 11(2):117-118.

37. Worthington K: Customer satisfaction in the emergency department. Emergency medicine clinics of North America 2004, 22(1):87-102. 
38. Delahaije DH, Dirksen CD, Peeters LL, Smits L: Anxiety and depression following preeclampsia or hemolysis, elevated liver enzymes, and low platelets syndrome. A systematic review. Acta obstetricia et gynecologica Scandinavica 2013, 92(7):746-761. 


\section{CHAPTER 2}

Cost-effectiveness of recurrence risk guided

care versus care as usual in women who

suffered from early-onset preeclampsia including HELLP syndrome in their previous pregnancy (the PreCare study)

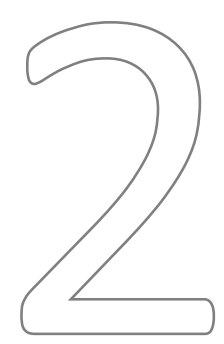

Denise HJ Delahaije, Sander MJ van Kuijk, Carmen D Dirksen, Simone JS Sep, Louis L Peeters, Marc E Spaanderman, Hein W Bruinse, Laura D de Wit-Zuurendonk, Joris AM van der Post, Johannes J Duvekot, Jim van Eyck, Mariëlle G van Pampus, Mark ABHM van der Hoeven, Luc J Smits

BMC Pregnancy Childbirth, 2010, 10:60 


\begin{abstract}
Background

Preeclampsia and HELLP syndrome may have serious consequences for both mother and fetus. Women who have suffered from preeclampsia or the HELLP syndrome, have an increased risk of developing preeclampsia in a subsequent pregnancy. However, most women will develop no or only minor complications. In this study, we intend to determine cost-effectiveness of recurrence risk guided care versus care as usual in pregnant women with a history of early-onset preeclampsia.
\end{abstract}

\title{
Methods/design
}

We developed a prediction model to estimate the individual risk of recurrence of earlyonset preeclampsia and the HELLP syndrome. In a before-after study, pregnant women with preeclampsia or HELLP syndrome in their previous pregnancy receiving care as usual (before introduction of the prediction model) will be compared with women receiving recurrence risk guided care (after introduction of the prediction model).

Eligible and pregnant women will be recruited at six university hospitals and seven large non-university tertiary referral hospitals in the Netherlands.

The primary outcome measure is the recurrence of early-onset preeclampsia or HELLP syndrome in women allocated to the regular monitoring group.

For the economic evaluation, a modelling approach will be used. Costs and effects of recurrence risk guided care with those of care as usual will be compared by means of a decision model. Two incremental cost-effectiveness ratios will be calculated: 1) cost per Quality Adjusted Life Year (mother unit of analysis) and 2) cost per live born child (child unit of analysis).

\section{Discussion}

This is, to our knowledge, the first study that evaluates prospectively the efficacy of a multivariable prediction rule for recurrent hypertensive disease in pregnancy. Results of this study could either be integrated into the current guideline on Hypertensive Disorders in Pregnancy, or be used to develop a new guideline. 


\section{Background}

\section{The disease}

Preeclampsia is defined as de novo development of hypertension ( $>140 / 90 \mathrm{mmHg}$ ) in combination with proteinuria after 20 weeks of gestation in pregnant women [1]. One of the most severe forms of this disease is called the HELLP syndrome, which is derived from the acronyms of the symptoms (Hemolysis, Elevated Liver enzymes and Low Platelets).

Preeclampsia (also termed early-onset preeclampsia) and HELLP syndrome may be life-threatening for both mother and child [2]. In the mother, these disorders predispose to premature cardiovascular disorders such as chronic hypertension, ischemic heart disease and stroke later in life [3]. Studies in children, born after preeclamptic pregnancies and who were relatively small at birth, have shown an increased risk of stroke in adult life, an increased risk of coronary heart disease and metabolic syndrome [4-6]. Preeclampsia and especially HELLP syndrome are perceived by patients and their partners as a highly traumatic life event, both during and after pregnancy [7].

In the United States and Europe, the prevalence of clinically relevant preeclampsia is approximately $2 \%$ of all pregnancies, with HELLP syndrome complicating preeclampsia approximately in 10 to $20 \%$ of the cases $[3,8]$. Maternal mortality rate in the Netherlands due to hypertensive disease during pregnancy amounts to 4.0 per 100,000 live births in 1993-2002 [9].

Although several risk factors have been identified, it is difficult to develop effective strategies for the prevention and treatment of these disorders [3]. Strategies applied nowadays are diverse and include antenatal surveillance, modification of lifestyle, dietary interventions and pharmacological therapy. In the last two decades, clinical guidelines for the management of preeclampsia and HELLP syndrome have been adopted in most developed countries [10-12].

Clinical management of preeclampsia or HELLP syndrome depends on the gestational age at onset, severity of symptoms, laboratory abnormalities, size and condition of the unborn infant, disease progression and response to symptomatic treatment.

Mild preeclampsia is usually managed expectantly, as opposed to the management of severe preeclampsia which is more pro-active consisting of the administration or magnesium sulphate, often combined with antihypertensives [13]. When gestational age is less than 34 weeks and both maternal and fetal condition are satisfactory, it is usually recommended to prolong pregnancy for at least 48 hours to benefit optimally from the enhancing effect of corticosteroid administration on fetal lung maturation. Fetal surveillance is an important component of the management. Delivery is the only 
causal treatment and therefore, the management of choice from 37 weeks' gestation onward $[14,15]$.

\section{The health care problem}

Although preeclampsia and HELLP syndrome are considered diseases of the first pregnancy, the risk of developing recurrent preeclampsia or HELLP syndrome is increased among parous women with preeclampsia and/or HELLP syndrome in their previous pregnancy. Sep et al. [16] conducted a literature search in order to identify prediction tests for recurrent disease. The recurrence rates reported vary from none to 31 percent for preeclampsia and from 3 percent to 7 percent for HELLP syndrome. Fortunately, the majority of women with a history of preeclampsia or HELLP syndrome have uncomplicated subsequent pregnancies.

To the best of our knowledge there is at this moment no consensus about the management of pregnant women with a history of early-onset preeclampsia or HELLP syndrome.

As a result, follow-up and counselling of these patients varies per centre, gynaecologist and patient, and is largely based on the perceived risk by the responsible care provider of recurrence of the disease in the next pregnancy and the level of anxiety of the patient and its resulting demand for care. Formerly preeclamptic patients are often subjected to various medical screening programmes to detect associated disorders, followed by additional exams by other specialists or the initiation of some management (postpartum evaluation). During a next pregnancy, care varies from regular surveillance by the gynaecologist to intensive surveillance and counselling in order to identify the onset of adverse pregnancy course as early as possible.

\section{Motivation and relevance for the study}

This lack of uniformity in the treatment of these patients asks for more standardisation, e.g. by providing care tailored to the individualised risk assessment.

Current policy may not be efficient. Since only a small percentage of these women develop early-onset recurrent disease in their next pregnancy, clinical management in the next pregnancy may benefit from subdividing these women into subgroups with or without increased risk. The care provided to these women can then be adjusted to their actual risk profile. Particularly former patients at low risk are expected to benefit from this type of risk stratification. Recurrence risk guided care could lead to a substantial reduction of (health care) costs and an increased quality of care.

We have recently developed a multiple-factor model for the prediction of recur-

rent early-onset preeclampsia and/or HELLP syndrome during the current pregnancy in 
women with a prior pregnancy complicated by preeclampsia and/or HELLP syndrome [17].

The PreCare study (pregnant women with previous preeclampsia: efficiency of care based on recurrence risk estimation), was designed to (1) estimate costeffectiveness of recurrence risk guided care versus care as usual for pregnant women with a previous pregnancy being complicated by preeclampsia or HELLP syndrome and (2) to validate the prediction model externally on the basis of the results of a prospective cohort and to update the prediction model if necessary. To this end, we will use the prediction model to differentiate the intensity of monitoring of pregnant women with a history of early-onset preeclampsia and/or HELLP syndrome. Patients are assigned to either the 'regular monitoring' or 'intensive monitoring' protocol. We will compare this strategy, referred to as recurrence risk guided care, with usual care for these patients in The Netherlands.

For this purpose, the following research questions were specified:

1. What are the effects of recurrence risk guided care versus care as usual for pregnant women with a previous preeclamptic pregnancy, on maternal and fetal morbidity and mortality?

2. What are the effects of recurrence risk guided care versus care as usual on specific and generic quality of life, anxiety, depression and development of posttraumatic stress?

3. What are the societal costs associated with the effects of recurrence risk guided care versus care as usual?

4. What is the cost-effectiveness of recurrence risk guided care versus care as usual?

\section{Development of prediction model}

Although earlier studies have identified individual predictive factors for recurrent preeclampsia, a combination of variables for the prediction of recurrent preeclampsia have not been explored until recently. A simple prepregnant prediction rule which includes several predictive factors was developed by Sep et al. [18]. Unfortunately, patient data were collected from a single hospital and the number of included patients was limited $(n=186)$.

We developed a prediction model to estimate the individual recurrence risk of recurrence of early-onset preeclampsia and/or the HELLP syndrome. We used data of 407 women with early-onset preeclampsia and/or the HELLP syndrome in their first pregnancy, who had undergone subsequent postpartum screening and who had a recorded consecutive ongoing pregnancy for which maternal and neonatal outcomes were available. Data were collected from four university hospitals (Maastricht Univer- 
sity Medical Centre, University Medical Centre Utrecht, Academic Medical Centre Amsterdam and University Medical Centre St. Radboud Nijmegen), and one tertiary referral hospital (Máxima Medical Centre Veldhoven).

Predictors of recurrent disease were preselected based on availability in the five different hospitals, previous literature and gynaecologists' expert opinion. Fasting circulating level of glucose measured at postpartum screening, gestational age at delivery of the first pregnancy, prior small-for-gestational-age newborn, chronic hypertension and maternal BMI before the second pregnancy proved to be the predictors within a logistic regression model [17].

Since a model tends to perform best in the derivation sample, called 'overfitting', we used bootstrapping techniques to internally validate the model. A shrinkage factor was computed to shrink the regression coefficients in order to get a more conservative risk estimate.

\section{Methods/design}

\section{Design}

The PreCare study uses a before-after design, in which outcomes and costs before the introduction of the prediction model (i.e. care as usual) are compared with outcomes and costs after introduction of the prediction model (i.e. recurrence risk guided care). This study consists of two consecutive phases. In the first phase of the study, 50 women receiving care as usual will be followed prospectively from their first pregnancyrelated outpatient visit until 3 months post partum. These 50 women will receive questionnaires in order to measure quality of life, anxiety, depression, posttraumatic stress and costs outside the hospital. Retrospective data of an additional group of 200 women who have been treated with care as usual in the past will be collected later on. Then the prediction rule will be introduced. In the second phase, 250 women will receive either protocolised regular monitoring or protocolised intensive monitoring depending on their risk of developing early-onset preeclampsia or HELLP syndrome during their current pregnancy, as estimated on the basis of the prediction model. In regular monitoring, pregnant women are monitored less intensively than in intensive monitoring. Regular monitoring and intensive monitoring are described in detail in the 'Monitoring protocols' section. Figure 1 summarises the design. 


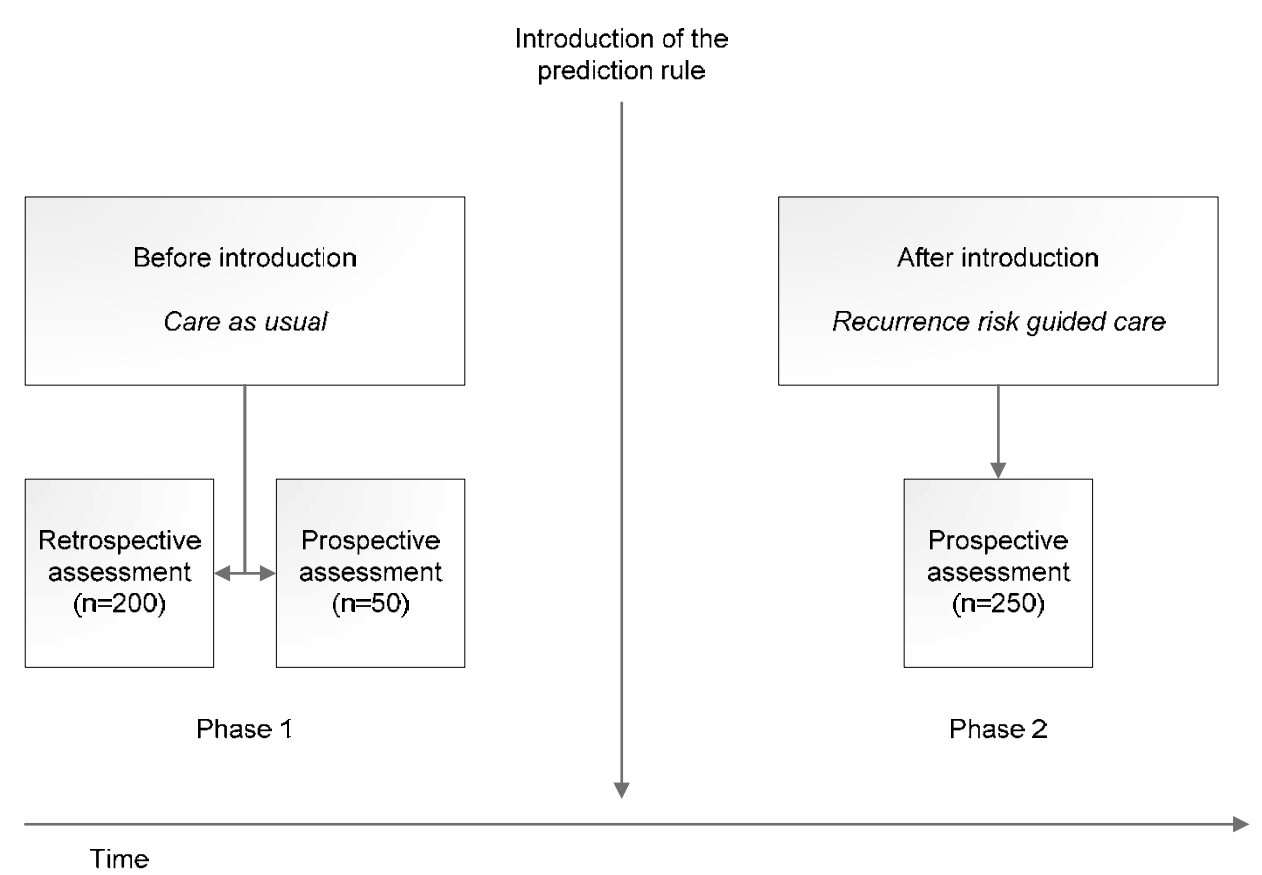

Figure 1: Design of the study

\section{Participants/eligibility criteria}

In phase 1 and 2, pregnant women (aged 18 years and older) with preeclampsia and/or HELLP syndrome in their previous pregnancy who have visited the outpatient clinic until 16+6 weeks of pregnancy are eligible for participation in the PreCare study.

Given these general eligibility criteria, two subgroups are distinguished in recurrence risk guided care (phase 2 ).

In women who gave birth after early-onset preeclampsia or HELLP syndrome until $33+6$ weeks gestational age in the previous pregnancy, the prediction model is applied in order to allocate the participants to one of the two monitoring protocols. The data which are required as input parameters for the prediction model should be available. These variables mostly come from the postpartum evaluation.

Women who gave birth between 34 and 36+6 weeks gestational age in the previous pregnancy face a relatively small risk of developing recurrent preeclampsia or HELLP syndrome, compared to women who gave birth before 34 weeks. Therefore, they all are allocated to the regular monitoring subgroup [19].

Women suffering from severe co-morbidity, such as diabetes mellitus, SLE, renal disease, cardiac disease or anti-phospholipids syndrome are excluded from the study as they will all receive (or have received where it concerns the retrospective patients) 
intensive surveillance. The current study will not include women who gave birth after 37 weeks gestational age.

The study population is recruited from six university hospitals and seven large non-university tertiary referral hospitals in the Netherlands: the Maastricht University Medical Centre, University Medical Centre St. Radboud Nijmegen, the Isala Clinics Zwolle, Erasmus Medical Centre Rotterdam, Academic Medical Centre Amsterdam, University Medical Centre Utrecht, Máxima Medical Centre Veldhoven, the University Medical Centre Groningen, Martini Hospital Groningen, Amphia Hospital Breda, Atrium Medical Centre Heerlen, Jeroen Bosch Hospital Den Bosch and Kennemer Hospital Haarlem.

\section{Recruitment procedures}

For the prospective assessment, 50 care as usual patients and 250 recurrence risk guided patients will be included in the study.

The research nurse or the gynaecologist of participating hospitals identifies eligible women by screening the appointment system of the outpatient clinic. After the pregnant woman has given informed consent for participation in the study, patients in phase 1 will be enrolled in care as usual whereas patients in phase 2 will be enrolled in recurrence risk guided care.

In recurrence risk guided care (phase 2), a simple web based risk calculator for the determination of recurrence risk and required intensity of surveillance has been developed and has been made accessible to gynaecologists and research nurses through the Internet.

Information about the recommended components of intensive monitoring and regular monitoring has been provided together with the risk calculator.

For the retrospective assessment, 200 patients who were treated according to care as usual will be used as a comparator group based on matching. Matching criteria will at least include maternal age (difference $<5$ years) and gestational age at delivery. Consequently, at study completion, there will be 250 care as usual patients who will be compared with 250 recurrence risk guided patients.

\section{Data collection}

For the prospective assessment ( $n=50$ care as usual; $n=250$ recurrence risk guided care), baseline demographics, past obstetric and medical history will be recorded in case report forms. Information is obtained on the condition of mother and child, infant weight, infant length, morbidity and mortality from the infant and maternal records. If applicable, details of the admittance of the child to the neonatal intensive care, high 
care or medium care unit will also be collected. All activities in the hospital will be documented until three months postpartum.

We will assess not only clinical outcomes, but also health-related quality of life by using the EQ-5D [20], anxiety by using the STAI [21], depression by using the Beck Depression Inventory [22] and posttraumatic stress by using the PTSD Symptom Scale [23]. The QoL questionnaires will be administered at 3-month intervals between baseline (before 20 weeks of pregnancy), at 29 weeks of pregnancy, ten days and three months postpartum. Costs outside the hospital, such as GP care, midwife and maternity care, productivity losses and out-of-pocket expenses will be obtained by means of a retrospective cost questionnaire with a recall period of 3 months. Long-term follow up of the women and children may be possible, but is not included in the current study.

For women enrolled in the retrospective assessment $(n=200)$, all data described above will be collected, except data on health-related quality of life, anxiety, depression, posttraumatic stress and costs outside the hospital.

\section{Monitoring protocols}

Development of the protocols

All gynaecologists of the participating centres were approached and they participated in a consensus process, during which the final protocols were designed. Nearly all were obstetricians specialised in hypertensive disorders of pregnancy. After reaching consensus, the protocols were distributed among all providers of care in the study.

The protocols

Figure 2 shows the process of recurrence risk guided care.

\section{Regular monitoring}

Women will visit the outpatient clinic 9 - 11 times: $(8,12), 16,20,24,28,32,36,38,39$ and 40 weeks gestational age. The visits at 16 and 20 weeks will be scheduled after the first trimester screening procedure and the routine ultrasound screening for structural malformations, respectively. If feasible, six of these eleven visits $(12,20,28,36,39,40)$ ought to be performed by the same obstetrician, whereas the remaining five (reassuring) visits $(8,16,24,32,38)$ may be performed by a nurse-practitioner or may be performed by the gynaecologist of the referring clinic (as of 32 weeks, in regular and intensive monitoring). 


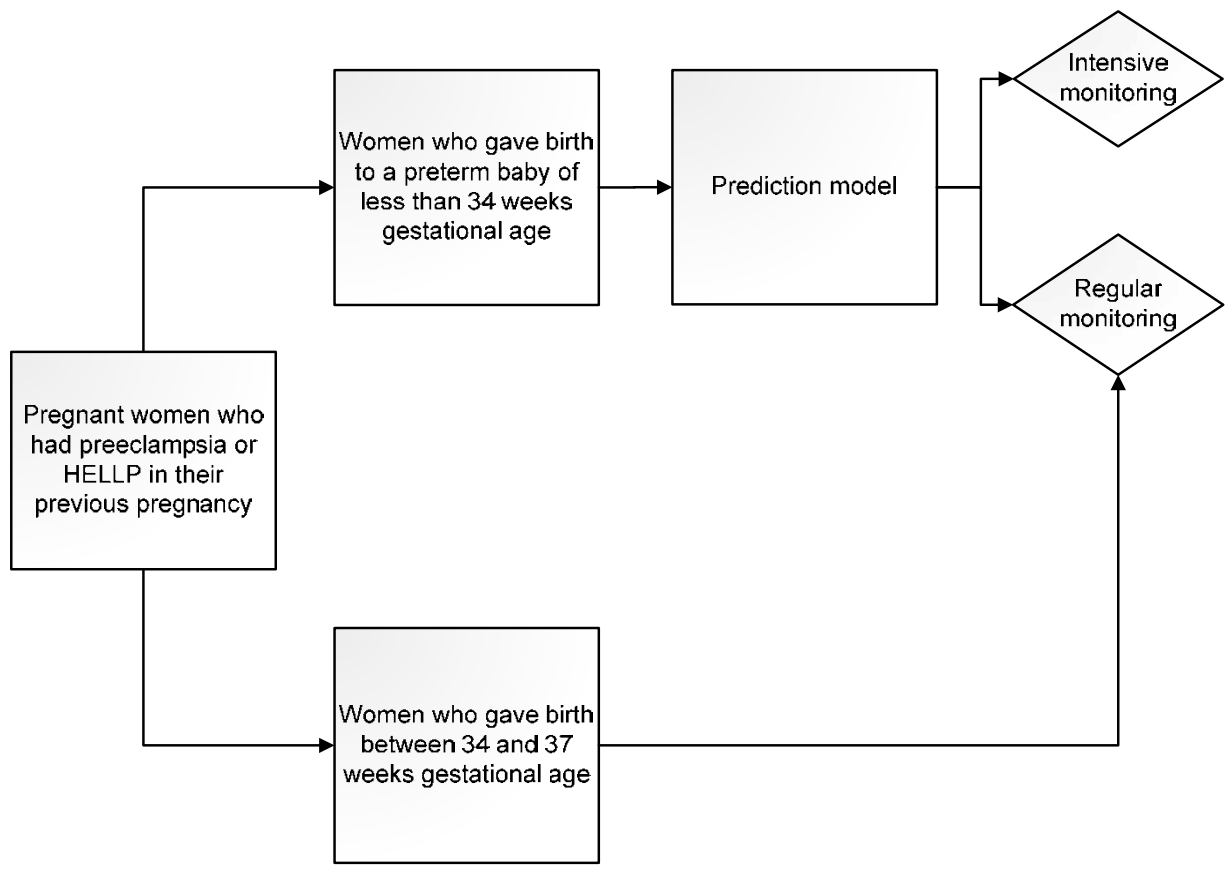

Figure 2: Recurrence risk guided care (phase 2)

\section{Intensive monitoring}

Women will visit the outpatient clinic 14 - 16 times: $(8,12), 16,18,20,22,24,26,28$, $30,32,34,36,38,39$ and 40 weeks gestational age.

At two occasions during an intensive monitoring pregnancy (16 and 20 weeks), the patient will undergo a series of diagnostic tests, which consist of:

1. Measurements in urine: (micro)albuminuria, creatinine, protein (protein serves to calculate the protein-to-creatinine ratio)

2. Measurements in blood: Haemoglobin, platelet count, mean platelet volume, creatinine, urate, CRP, glucose, fibronectin, Flt-1, endoglin

3. $24 \mathrm{~h}$ blood pressure monitoring. This assessment is optional (not obligatory)

4. Assessment of the Doppler flow velocity profile in both uterine arteries (at 20 weeks)

Activities other than, or additional to those described in the regular monitoring and intensive monitoring protocols (such as intercurrent admissions, additional outpatient visits or diagnostic testing, or telephone/e-mail contacts) will be registered. 


\section{Study outcomes}

Recurrence risk guided care will be compared with care as usual with respect to several outcomes.

Primary outcome is the occurrence of either early-onset preeclampsia or HELLP syndrome. Early-onset preeclampsia and early-onset HELLP syndrome are defined according to the criteria of the International Society for the Study of Hypertension in Pregnancy with the criterion of delivery before 34 weeks after the last menstrual period [24]. Secondary clinical outcome measures are: gestational age of onset of preeclampsia, eclampsia, HELLP syndrome, intrauterine growth restriction, caesarean section, admission to the neonatal intensive care unit (NICU), gestational age at delivery and maternal/infant mortality. Other secondary outcome measures are societal costs, quality of life, anxiety, depression, development of posttraumatic stress, satisfaction with treatment, protocol adherence and cost-effectiveness.

\section{Statistical analysis}

Sample size

Sample size calculation was based on the expected fraction of women assigned to regular monitoring either on the basis of the prediction rule or gestational age at the time of previous delivery (67\%), the expected failure rate within this category ( $2 \%)$, a type I error (one-sided) of 0.05 and a type II error of 0.2 . In order to be able to exclude failure rates of $5 \%$ or more, 150 patients assigned to regular monitoring are needed. This means that results of about 225 patients in the recurrence risk based care are needed. The estimated number of 250 allows for drop out and incomplete data of 25 patients.

\section{Data analysis}

The primary analysis concerns the incidence of recurrent early-onset preeclampsia or HELLP syndrome in both groups (recurrence risk guided care versus care as usual). Analyses will be adjusted for potential confounders (including demographic factors). Confidence intervals will be adjusted by means of multilevel analysis (hospital).

We will also analyse whether the new patient data indicate the need for an update of the predictive model. For this purpose, we will first compare the characteristics of the original population used for model development with the new population, the so-called "validation population". For example, the incidence of early-onset preeclampsia may differ between the original and validation population. It is also possible that the latter population has a different case-mix (i.e. differences in distributions of the predictors in the population), because, e.g., more hospitals are included. In 
addition, different predictor-outcome associations and additional predictors, that are not included in the model but are either more or less frequent in the new population, could play a role. We will also assess model performance in the validation population by comparing sensitivity, specificity, positive and negative predictive values and discrimination and calibration compared to the performance in the derivation population.

If the analysis should indicate lower accuracy than expected, we will analyse to what extent all these factors indeed affect the model's accuracy and update the model to obtain adequate accuracy. Updating of the model will be done by means of recalibration (step 1) and model revision (step 2) [25-27].

\section{Economic evaluation}

A cost-effectiveness analysis will be performed from a societal perspective, comparing the costs and effects of recurrence risk guided care versus care as usual. The time horizon of the study is 9 months (3 months amenorrhea - 3 months post partum). Discounting is not relevant given the short time horizon.

The cost analysis will be performed according to the Dutch guidelines for cost calculations [28]. All hospital resource use and costs associated with care for pregnant women and their newborns will be calculated from study entry until 3 months post partum and include costs such as outpatient visits and hospital admissions. Cost prices will be obtained from participating hospitals. If prices are not readily available, directive prices will be used [28] or additional calculations will be made. Costs in the analysis also include direct non-health care costs (travel costs) and indirect costs (productivity loss).

Effect parameters are clinical outcomes, health related quality of life, anxiety, depression and posttraumatic stress.

Currently, no economic evaluation methods are available that integrate health outcomes of both mother and child into a single outcome measure. However, preeclampsia may have health effects for both the pregnant women and her (unborn) child. Therefore, the main cost-effectiveness analysis will involve calculating two incremental cost-effectiveness ratios (ICERs) expressing 1) the cost per Quality Adjusted Life Year (QALY, mother unit of analysis) and 2) the cost per live born infant (child unit of analysis). To this end, a decision model will be developed. Modelling in economic evaluation is considered useful for example when experimental observations from a trial are missing, which in this study applies to some parameters in the care as usual group [29, 30]. 


\section{Ethical considerations}

This study has been approved by the ethical committee of the University Hospital Maastricht (Ref.no. MEC 07-2-078). A total of six academic and seven non-academic hospitals participate in the study; all of them have completed their obligatory feasibility assessment procedure successfully. Informed consent is being obtained from all patients prior to enrolment into the study.

\section{Discussion}

This study is expected to yield information on health outcomes and costs of adjusting the level of care to the estimated probability of recurrent preeclampsia. The results can provide a basis for more uniform (and evidence-based) guidelines for care for formerly preeclamptic women and possibly lead to more cost-effective provision of health care. With respect to health care costs, it is expected that mean costs per patient will decrease as a result of a reduction in intensive maternal and fetal surveillance. Potential savings can be even higher, since the majority of women assigned to regular monitoring may be adequately served by care provided by in-hospital midwifes [31].

The close cooperation with many centres enables us to reach a representative study population of pregnant women who have experienced preeclampsia in their previous pregnancy, which enhances the applicability of the results to all former preeclamptic women. Results of this study will be disseminated by means of presentations at scientific meetings and peer-reviewed publications. Study outcomes will also be communicated directly to the NVOG (Dutch Association of Obstetrics and Gynaecology), KNOV (Royal Dutch Association of Midwives), and the Dutch HELLP syndrome foundation. In addition, we will cooperate with the NVOG in order to produce recommendations for the formulation of guidelines. The recommendations could either be integrated into the current guideline on Hypertensive Disorders in Pregnancy, or be used to develop a new guideline. The results of this study will be used to standardise the postpartum evaluation of women with a recent history of preeclampsia or HELLP syndrome. It is expected that the number of tests in the postpartum evaluation will not only be reduced, but also synchronised.

A randomised controlled trial design is usually preferred over any other design. However, for this study such a design was not considered applicable. A before-after study was chosen (instead of a fully prospective, randomised study) because of the risk of care as usual being contaminated with the regular monitoring and intensive monitoring protocols. Because current care as usual is not standardised, and blinding of the participating gynaecologists with respect to predictive factors for recurrent disease is 
practically unfeasible, a randomised design could reduce the contrast between study arms and thereby threaten the validity of the results.

As the care provided to women assigned to regular monitoring is less intense, a possible consequence may be that the detection of clinical signs of preeclampsia or HELLP syndrome is somewhat later than in the current care as usual approach. However, we do not expect this to lead to adverse maternal or fetal outcomes, because the prediction model suggests that the recurrence rate in this group is low $(<1 \%)$ whereas there is also increased alertness for early signs of pregnancy complications in women with a history of preeclampsia or HELLP syndrome.

In summary, the PreCare study is designed to provide information on whether recurrence risk guided care is a worthwhile strategy compared to current care for pregnant women who suffered from preeclampsia or HELLP syndrome during their previous pregnancy.

\section{References}

1. Davison JM, Homuth V, Jeyabalan A, Conrad KP, Karumanchi SA, Quaggin S, Dechend R, Luft FC: New aspects in the pathophysiology of preeclampsia. Journal of the American Society of Nephrology : JASN 2004, 15(9):2440-2448.

2. Barden A: Pre-eclampsia: contribution of maternal constitutional factors and the consequences for cardiovascular health. Clinical and experimental pharmacology \& physiology 2006, 33(9):826-830.

3. Meads CA, Cnossen JS, Meher S, Juarez-Garcia A, ter Riet G, Duley L, Roberts TE, Mol BW, van der Post $J A$, Leeflang MM et al: Methods of prediction and prevention of pre-eclampsia: systematic reviews of accuracy and effectiveness literature with economic modelling. Health Technol Assess 2008, 12(6):iii-iv, 1-270.

4. Osmond C, Kajantie E, Forsen TJ, Eriksson JG, Barker DJ: Infant growth and stroke in adult life: the Helsinki birth cohort study. Stroke; a journal of cerebral circulation 2007, 38(2):264-270.

5. Eriksson JG, Forsen T, Tuomilehto J, Osmond C, Barker DJ: Early growth and coronary heart disease in later life: longitudinal study. BMJ 2001, 322(7292):949-953.

6. Barker DJ, Martyn CN, Osmond C, Hales CN, Fall CH: Growth in utero and serum cholesterol concentrations in adult life. BMJ 1993, 307(6918):1524-1527.

7. Sibai B, Dekker G, Kupferminc M: Pre-eclampsia. Lancet 2005, 365(9461):785-799.

8. Haram K, Svendsen E, Abildgaard U: The HELLP syndrome: clinical issues and management. A Review. BMC pregnancy and childbirth 2009, 9:8.

9. Schutte JM, Schuitemaker NW, van Roosmalen J, Steegers EA, Dutch Maternal Mortality C: Substandard care in maternal mortality due to hypertensive disease in pregnancy in the Netherlands. BJOG : an international journal of obstetrics and gynaecology 2008, 115(6):732-736.

10. NVOG: Richtlijn 6 - Hypertensieve aandoeningen in de zwangerschap. In.; 2005.

11. Magee LA, Helewa M, Moutquin JM, von Dadelszen P, Hypertension Guideline C, Strategic Training Initiative in Research in the Reproductive Health Sciences S: Diagnosis, evaluation, and management of the hypertensive disorders of pregnancy. Journal of obstetrics and gynaecology Canada : JOGC = Journal d'obstetrique et gynecologie du Canada : JOGC 2008, 30(3 Suppl):S1-48. 
12. Societe francaise d'anesthesie et de $r$, College national des gynecologues et obstetriciens $f$, Societe francaise de medecine $p$, Societe francaise de $n$ : [Multidisciplinary management of severe pre-eclampsia (PE). Experts' guidelines 2008. Societe francaise d'anesthesie et de reanimation. College national des gynecologues et obstetriciens francais. Societe francaise de medecine perinatale. Societe francaise de neonatalogie]. Annales francaises d'anesthesie et de reanimation 2009, 28(3):275-281.

13. Berkow R, Beers MH, Fletscher AJ: Merck Manual Medisch handboek. Houten/Diegem: Bohn Stafleu Van Loghum; 2000.

14. Williams J, Mozurkewich E, Chilimigras J, Van De Ven C: Critical care in obstetrics: pregnancy-specific conditions. Best practice \& research Clinical obstetrics \& gynaecology 2008, 22(5):825-846.

15. Koopmans CM, Bijlenga D, Groen H, Vijgen SM, Aarnoudse JG, Bekedam DJ, van den Berg PP, de Boer K, Burggraaff JM, Bloemenkamp KW et al: Induction of labour versus expectant monitoring for gestational hypertension or mild pre-eclampsia after 36 weeks' gestation (HYPITAT): a multicentre, open-label randomised controlled trial. Lancet 2009, 374(9694):979-988.

16. Sep S, Smits L, Prins M, Peeters L: Prediction tests for recurrent hypertensive disease in pregnancy, a systematic review. Hypertension in pregnancy : official journal of the International Society for the Study of Hypertension in Pregnancy 2010, 29(2):206-230.

17. van Kuijk SM, Nijdam ME, Janssen KJ, Sep SJ, Peeters LL, Delahaije DH, Spaanderman M, Bruinse HW, Franx A, Bots ML et al: A model for preconceptional prediction of recurrent early-onset preeclampsia: derivation and internal validation. Reprod Sci 2011, 18(11):1154-1159.

18. Sep SJ, Smits LJ, Prins MH, Spaanderman ME, Peeters LL: Simple prepregnant prediction rule for recurrent early-onset hypertensive disease in pregnancy. Reprod Sci 2009, 16(1):80-87.

19. Zhang J, Troendle JF, Levine RJ: Risks of hypertensive disorders in the second pregnancy. Paediatric and perinatal epidemiology 2001, 15(3):226-231.

20. Anonymus: EuroQoL-a new facility for the measurement of health-related quality of life. The EuroQol Group. Health Policy 1990, 16:199-208.

21. Spielberger CD, Gorsuch RL, Lushene RE: STAI Manual for the State-trait Anxiety Inventory. Palo Alto, CA: Consulting Psychologists Press; 1970.

22. Beck AT, Ward CH, Mendelson M, Mock J, Erbaugh J: An inventory for measuring depression. Archives of general psychiatry 1961, 4:561-571.

23. Foa EB, Riggs DS, Rothbaum B: Reliability and validity of a brief instrument for assessing post traumatic stress disorder. . JTS 1993, 6:459-473.

24. Brown MA, Lindheimer MD, de Swiet M, Van Assche A, Moutquin JM: The classification and diagnosis of the hypertensive disorders of pregnancy: statement from the International Society for the Study of Hypertension in Pregnancy (ISSHP). Hypertension in pregnancy : official journal of the International Society for the Study of Hypertension in Pregnancy 2001, 20(1):IX-XIV.

25. Van Houwelingen HC, Thorogood J: Construction, validation and updating of a prognostic model for kidney graft survival. Statistics in medicine 1995, 14(18):1999-2008.

26. van Houwelingen HC: Validation, calibration, revision and combination of prognostic survival models. Statistics in medicine 2000, 19(24):3401-3415.

27. D'Agostino RB, Sr., Grundy S, Sullivan LM, Wilson P, Group CHDRP: Validation of the Framingham coronary heart disease prediction scores: results of a multiple ethnic groups investigation. JAMA : the journal of the American Medical Association 2001, 286(2):180-187.

28. Oostenbrink JB, Bouwmans CAM, Koopmanschap MA, Rutten FFH: Handleiding voor kostenonderzoek: Methoden en standaard kostprijzen voor economische evaluaties in de gezondheidszorg. . Rotterdam: College voor zorgverzekeringen; 2004.

29. Buxton MJ, Drummond MF, Van Hout BA, Prince RL, Sheldon TA, Szucs T, Vray M: Modelling in economic evaluation: an unavoidable fact of life. Health economics 1997, 6(3):217-227.

30. Brennan A, Akehurst R: Modelling in health economic evaluation. What is its place? What is its value? PharmacoEconomics 2000, 17(5):445-459. 
31. De thuisbevalling in Nederland, 1995-2002. Rapportage over de jaren 2001-2002 van PRN/TNO. [http://www.perinatreg.nl/uploads/153/99/Rapportage_Thuisbevalling_2001-2002.pdf] 


\section{CHAPTER 3}

\section{Care-as-usual provided to formerly preeclamptic women in the Netherlands in the next pregnancy: health care consumption, costs and pregnancy outcome}

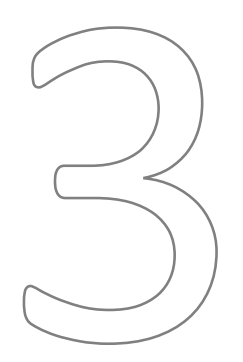

Denise HJ Delahaije, Luc J Smits, Sander MJ van Kuijk, Louis L Peeters, Johannes J Duvekot, Wessel Ganzevoort, Martijn A Oudijk, Mariëlle G van Pampus, Hubertina CJ Scheepers, Marc E Spaanderman, Carmen D Dirksen for the PreCare study group

Eur J Obstet Gynecol Reprod Biol, 2014, 179:240-5 


\begin{abstract}
Objective

To explore hospital costs by pregnant women with a history of early-onset preeclampsia or HELLP syndrome, managed according to customary, but non-standardized prenatal care, by relating maternal and child outcome to maternal health care expenditure.
\end{abstract}

\title{
Study design
}

This was a cohort study, in women of 18 years or older who suffered from early-onset preeclampsia or HELLP syndrome in their previous pregnancy $(n=104)$. We retrieved data retrospectively from hospital information systems and medical records of patients who had received customary, non-standardized prenatal care between 1996 and 2012. Our analyses focused on the costs generated between the first antenatal visit at the outpatient clinic and postpartum hospital discharge. Outcome measures were hospital resource use, costs, maternal and child outcome (recurrence of preeclampsia or HELLP syndrome, incidence of eclampsia, gestational age at delivery, intrauterine fetal demise, small-for-gestational-age birth and low 5 minutes' Apgar score). We used linear regression analyses to evaluate whether maternal and child outcome and baseline characteristics correlated with hospital costs.

\section{Results}

Maternal hospital costs per patient averaged $€ 8,047$. Main cost drivers were maternal admissions and outpatient visits, together accounting for $80 \%$ of total costs. Primary cost drivers were preterm birth and recurrent preeclampsia or HELLP syndrome.

\section{Conclusion}

Hospital costs in the next pregnancy of formerly preeclamptic women varied widely with over $70 \%$ being medically unexplainable. The results of this study support the view that care standardization in these women can be expected to improve costs and efficacy of care without compromising outcome. 


\section{Introduction}

Preeclampsia (PE) affects about 2-5\% and the syndrome of Hemolysis, Elevated Liver enzymes and Low Platelets (HELLP) $0.5 \%$ of all pregnancies $(1,2)$. Women with a history of PE or HELLP are at increased risk of recurrence in their next pregnancy $(1,2)$. Women who experienced early-onset PE in the first pregnancy have a 6.6-fold higher risk of recurrence in their next pregnancy than women who completed a normotensive first pregnancy (3). Therefore, gynecologists often provide extensive follow-up and counseling to these women, both postpartum and during their next pregnancy. However, only $7 \%$ of these former patients will actually develop a recurrent early-onset PE in their next pregnancy (2). Therefore, current clinical management may be excessive in most former patients (4-6). Nowadays, the challenge of rapidly rising health care costs asks for close scrutiny on costs and benefits of all medical treatments. At this moment, there are no evidence-based and standardized clinical practice guidelines for the management of pregnant women with a history of early-onset PE or HELLP. As a consequence, the intensity of follow-up can be expected to vary per center, gynecologist and patient, influenced by subjective factors, such as the perceived recurrence risk in the next pregnancy and the patient's neuroticism, anxiousness and associated demand for care $(7,8)$. This practice variation is referred to as care-as-usual (CAU): "the full spectrum of patient care practices in which clinicians have the opportunity to individualize care" (9). Describing CAU is an essential first step in understanding and identifying potential options to ameliorate the costs and efficiency of care.

This study aims to describe the hospital costs of CAU in a cohort of pregnant women with a previous pregnancy complicated by early-onset PE/HELLP in the Netherlands, and whether these costs relate to pregnancy outcome.

\section{Material and methods}

\section{Study design}

This study is part of a multicenter "before-after" study (the PreCare study) designed to compare effects and costs of recurrence risk guided care (RGC) with those of CAU (10). In RGC (the 'after' part of the study), pregnant women were assigned to either Medium or High Care, depending on their anticipated risk to develop recurrent PE/HELLP, estimated at booking using a prediction model (11). In CAU (the 'before' part of the study) gynecologists were asked to treat their patients as they deemed appropriate. All women in our study received low-dose aspirin from 12 to 37 weeks pregnancy, a practice which became universally accepted in the Netherlands shortly after the CLASP 
study (1994). The CLASP study provided evidence for a low-dose aspirin -taken from early pregnancy onwards- to lower the risk of developing PE in women at increased risk of developing PE (12). This practice has not changed since. For this study we used data retrieved from hospital information systems and medical records of patients who had received CAU between 1996 and 2012 in six university and three non-university hospitals in the Netherlands: Maastricht University Medical Center, Erasmus Medical Center Rotterdam, Academic Medical Center Amsterdam, Radboud University Medical Center Nijmegen, University Medical Center Groningen, University Medical Center Utrecht, Atrium Hospital Heerlen, Onze Lieve Vrouwe Hospital Amsterdam, Amphia Hospital Breda.

\section{Participants}

Pregnant women of over 18 years of age, with their previous pregnancy being complicated by PE and/or the HELLP syndrome, requiring pregnancy termination before 37 weeks, were eligible for enrolment. We excluded women with severe co-morbidity (diabetes mellitus, systemic lupus erythematosus, renal disease and cardiac disease or the anti-phospholipid syndrome).

\section{Estimation of hospital resource use}

Hospital resource use was assessed by retrieving data from hospital information systems and medical records. All cost-generating activities in the hospital (maternal admissions, outpatient visits, maternal lab tests, mode of delivery, etc.) were registered at the patient level and recorded online in case report forms (CRFs). We classified intensive care and obstetrical ward admissions separately and categorized childbirth depending on whether or not the delivery was induced and whether or not delivery required termination by forceps/vacuum extraction or cesarean section. Variation in resource use was then determined and evaluated in relation to baseline characteristics and maternal and child outcome.

\section{Estimation of unit costs}

We performed cost calculations using the Dutch manual for cost research in health care, a methodological reference for costing studies in the Netherlands (13). The cost analysis was performed from the hospital perspective and covered the interval from conception until maternal postpartum discharge from hospital. Unit prices are presented in 2011 Euros. If necessary, costs were adjusted to the 2011 price level using the consumer price index (14). In order to assess whether a time effect was present in 
the data, we tested the calendar year from which the data were retrieved (1996-2012) for its association with maternal hospital costs. For each individual patient, we calculated a 'time trend variable' by subtracting year of delivery from 1996.

\section{Pregnancy outcome}

Baseline characteristics of participants, such as obstetric and medical histories, and maternal and child outcome were recorded in CRFs. We considered the following types of adverse maternal outcome to be relevant for analysis: recurrent PE/HELLP, requiring pregnancy termination before the 34th week, preterm birth (delivery $<37$ weeks) and recurrence of PE/HELLP/eclampsia, irrespective of gestational age. Meanwhile, intrauterine fetal demise (IUFD), low birth weight (birth weight centile $<10 \%$ ) and 5-min. Apgar score (below 7) were considered adverse child outcome.

\section{Details of ethical approval}

This study was approved by the medical-ethical committee of the University Hospital Maastricht (Ref.no. MEC 07-2-078). All hospitals successfully completed their obligatory feasibility assessment procedure.

\section{Statistical analysis}

Prior to analysis, we checked completeness and validity of our dataset. Two of the authors (DD and SvK) contacted the research nurses in the participating hospitals to maximize the effort to retrieve missing data or to correct identified inconsistencies in the data. If missing values could not be retrieved, we performed regression imputation (except for baseline characteristics, Table 1). If the total number of CRF-registered outpatient visits was unrealistically low (below 5), the number was considered missing, and also imputed by single imputation (15).

We used simple statistics, such as frequencies, percentages, means and standard deviations (SDs) to describe population characteristics at baseline, resource use (use of hospital care), hospital costs, inter-hospital variation and maternal and child outcome. First, in order to get insight into the crude associations between maternal and child outcome on the one hand and maternal hospital costs on the other hand, all variables were examined by univariate regression analyses for their relation with maternal hospital costs. In the second step, all these variables were included in a multivariate linear regression model using a backward selection procedure $(\alpha=0.10)$ with total maternal hospital costs as dependent variable. In a first additional analysis, we applied the same linear regression model (using a backward selection procedure, $\alpha=0.10$ ), including only 
the subgroup of patients who had not developed recurrent PE/HELLP ( $n=76)$, to enable identification of other factors also contributing to maternal hospital costs, in the absence of recurrent PE/HELLP. This separate analysis was expected to increase the discriminatory power of our study as PE/HELLP recurrence requiring hospital admission tended to dominate total hospital costs. Collinearity between the variables in the model was assessed by examining the tolerance and variance inflation factor for each independent variable. We verified normality of the residuals using a normal probability plot of the residuals.

For all the analyses, we used the Statistical Package for the Social Sciences 20.0 (SPSS Inc., Chicago, USA) and Microsoft Excel 2010 (Microsoft Corp., Redmond, CA).

\section{Results}

\section{Study population}

A total of 104 patients fulfilled the eligibility criteria. Table 1 lists the baseline characteristics of the study population. For 64 women (61.5\%) we were unable to retrieve from the records whether or not they had preexisting hypertension. In addition, in two-third of the participants $(n=70)$, we were unable to retrieve the birth weight data of the infants born after the complicated pregnancy. Therefore, we missed in $>50 \%$ of the participants $(n=59)$ information on whether or not they had given birth to an SGA infant in that pregnancy.

\section{Hospital resource use and associated costs}

Table 2 lists data on prenatal care, childbirth, and hospital admission. The mean length of hospital stay for those requiring hospitalization was 8 days $(S D=6.72)$. About half of the women (51\%) required cesarean birth, whereas $\approx 30 \%$ delivered spontaneously.

Patients visited the outpatient clinic on average 17 times. Total costs averaged $€ 8,047$ with major contributors being maternal admissions (57\%) and outpatient visits (26\%). 
Table 1: Characteristics of the study population

Maternal characteristics at conception of the target pregnancy

Maternal age at conception, years (SD)

$30.7(5.0)$

Body mass index, $\mathrm{kg} / \mathrm{m}^{2}$ (SD)

$27.3(6.1)$

Primiparous, $\mathrm{n}(\%)$

$93(89.4)$

Multiparous, n (\%)

$11(10.6)$

\section{Characteristics of previous pregnancy}

Gestational age at delivery (weeks)

$31.1(3.8)$

< 34 weeks, $\mathrm{n}(\%)$

78 (75)

34 to $36+6$ weeks, $n$ (\%)

26 (25)

PE, n (\%)

95 (91.3)

HELLP syndrome, $\mathrm{n}$ (\%)

59 (56.7)

Eclampsia, $\mathrm{n}(\%)$

5 (4.8)

IUFD, $\mathrm{n}(\%)$

14 (13.5)

Birth weight, g (SD)

$1408(765)$

Data are given as mean \pm SD or as percentages 
Table 2: Mean costs, resource use, subtotal and total costs in Euros for care-as-usual (CAU)

\begin{tabular}{|c|c|c|c|c|}
\hline \multirow[t]{2}{*}{ All patients } & \multirow[t]{2}{*}{$\begin{array}{l}\text { Cost per unit, } \\
€(2011)^{\mathrm{a}}\end{array}$} & \multirow[t]{2}{*}{$\begin{array}{l}\text { Volumes of } \\
\text { use for CAU }\end{array}$} & \multicolumn{2}{|c|}{$\begin{array}{l}\text { Costs for CAU per patient, } \\
\text { means }(€)(n=104)\end{array}$} \\
\hline & & & Costs $^{\mathrm{b}}$ & $\%$ of total \\
\hline \multicolumn{5}{|l|}{ Direct health care costs mother } \\
\hline Tests and procedures, blood ${ }^{c}$ & Various/Procedure $^{1}$ & n.a. & 92.59 (106.59) & $1.15 \%$ \\
\hline Tests and procedures, urine ${ }^{c}$ & Various/Procedure ${ }^{1}$ & n.a. & $40.83(59.13)$ & $0.51 \%$ \\
\hline Outpatient clinic gynecologist & $66.32-133.68 /$ Visit $^{2}$ & $16.85(7.46)$ & $2,066.11(1,048.95)$ & $25.68 \%$ \\
\hline Fetal ultrasound & 35.99/Determination ${ }^{1}$ & $6.39(5.30)$ & $229.90(190.55)$ & $2.85 \%$ \\
\hline $\begin{array}{l}\text { Automated blood pressure } \\
\text { monitoring }\end{array}$ & 1.52/Determination ${ }^{1}$ & $9.73(15.79)$ & $14.79(24.00)$ & $0.18 \%$ \\
\hline $\begin{array}{l}\text { Maternal admission peripartum } \\
\text { period }\end{array}$ & $\begin{array}{l}450.79-595.87- \\
2,262.24 / \text { day }^{2}\end{array}$ & $8.01(6.72)$ & $4,616.45(3,995.88)$ & $57.37 \%$ \\
\hline \multicolumn{5}{|l|}{ Mode of delivery } \\
\hline Spontaneous & $766.27 /$ Procedure $^{1}$ & 0.298 & 228.5 & \\
\hline Spontaneous after induction & 781.21/Procedure ${ }^{1}$ & 0.029 & 22.66 & \\
\hline Instrumental & 786.81/Procedure ${ }^{1}$ & 0.048 & 37.77 & \\
\hline Instrumental after induction & $801.81 /$ Procedure $^{1}$ & 0.038 & 30.47 & \\
\hline Cesarean section & $1134.60 /$ Procedure $^{1}$ & 0.51 & 578.65 & \\
\hline $\begin{array}{l}\text { Cesarean section after } \\
\text { induction }\end{array}$ & $1149.60 /$ Procedure $^{1}$ & 0.077 & 88.52 & \\
\hline $\begin{array}{l}\text { Cesarean section after } \\
\text { vacuum/forceps }\end{array}$ & 1921.41/Procedure ${ }^{1}$ & 0 & & \\
\hline Subtotal mode of delivery & & & 986.25 (180.10) & $12.26 \%$ \\
\hline Total costs mother & & & $8,046.92(4,048.63)$ & $100 \%$ \\
\hline
\end{tabular}

Data are given as mean \pm SD

a) Source of unit price; 1) Dutch manual (Oostenbrink et al., 2004); 2) Dutch manual (Hakkaart-van Roijen et al., 2010); b) Total costs may not exactly be the product of cost per unit and volumes of use due to rounding; c) As tests and procedures varied in type and costs, n.a. (not applicable) is shown for the volumes of use.

\section{Practice variation}

The number of outpatient visits between centers varied widely, though, without a consistent pattern (ANOVA, $p=0.662$ ). Although the mean number of visits to the outpatient clinic in the 9 participating hospitals ranged from 13 to 20 (Table 3), individual cases varied as much as from 5 to 37 visits. 
Table 3: Practice variation between centers with regard to outpatient visits

\begin{tabular}{lccll}
\hline Center & N & Minimum & Maximum & Mean (SD) \\
\hline A & 18 & 5 & 37 & $18(11.6)$ \\
B & 11 & 8 & 37 & $18(8.3)$ \\
C & 13 & 7 & 26 & $13(4.9)$ \\
D & 7 & 12 & 23 & $16(3.7)$ \\
E & 3 & 6 & 28 & $19(11.9)$ \\
F & 20 & 9 & 28 & $17(4.6)$ \\
G & 7 & 6 & 20 & $15(4.9)$ \\
H & 9 & 12 & 37 & $19(7.5)$ \\
I & 16 & 8 & 37 & $15(7.3)$ \\
\hline University & 87 & 5 & 37 & $16(7.8)$ \\
Non-university & 17 & 6 & 28 & $16(5.8)$ \\
\hline
\end{tabular}

\section{Pregnancy outcome of mother and child}

Table 4 lists maternal and child outcome variables. All pregnancies were singleton, resulting in the birth of 51 boys and 53 girls, including 5 perinatal deaths.

Table 4: Maternal and child outcome of the target pregnancy

\begin{tabular}{ll}
\hline Maternal outcome & $37.1(4.3)$ \\
Gestational age at delivery, weeks (SD) & $18(17.3)$ \\
$<34$ weeks, n (\%) & $9(8.7)$ \\
34 to 36+6 weeks, n (\%) & $77(74.0)$ \\
$\quad$ after 37 weeks, n (\%) & $7(6.7)$ \\
Recurrent early-onset PE, n (\%) & $16(15.4)$ \\
Recurrent PE (all cases), n (\%) & $4(3.8)$ \\
Recurrent early-onset HELLP, n (\%) & $5(4.8)$ \\
Recurrent HELLP (all cases), n (\%) & $2(1.9)$ \\
Eclampsia, n (\%) & \\
Child outcome & \\
IUFD, n (\%) & $5(4.8)$ \\
Birth weight, g (SD) & $2,833(1,094)$ \\
SGA, n (\%) & $26(25)$ \\
Five minutes' Apgar score (SD) & $9(1.2)$ \\
\hline
\end{tabular}

Data are given as mean \pm SD or as percentages 


\section{Relation between mother and child characteristics and maternal costs}

We examined how maternal hospital costs related to maternal and child characteristics. We made a distinction between pre-existing baseline characteristics (including features of the index pregnancy), and outcome of the target pregnancy (until postpartum discharge). In univariate regression analyses, maternal hospital costs in older women were higher for each variable $(p=0.036)$. The costs increased with increasing parity and gravidity ( $p=0.014$ and $p=0.010$, respectively). Hospital costs for patients who developed recurrent PE/HELLP were markedly higher than those for their counterparts not developing a recurrence $(€ 3,720 ; p=0.000)$. We also observed an inverse relation of hospital costs with gestational age at birth and birth weight (Table 5).

Table 5: Univariate regression analyses examining the relation between mother and child characteristics and maternal hospital costs, including all women $(n=104)$

\begin{tabular}{lllll}
\hline Variable & $\mathrm{n}$ & $\mathrm{B}(\mathrm{SE})$ & $p$ & $\mathrm{R}^{2}$ \\
\hline $\begin{array}{l}\text { Maternal characteristics } \\
\text { Baseline (before and index pregnancy) }\end{array}$ & & & & \\
Age at conception (years) & 104 & $165(78)$ & $0.036^{*}$ & 0.043 \\
Gravidity & 104 & $1111(443)$ & $0.014^{*}$ & 0.058 \\
Parity & 104 & $2157(820)$ & $0.010^{*}$ & 0.064 \\
BMI & 104 & $-96(78)$ & 0.221 & 0.015 \\
Gestational age delivery index pregnancy (weeks) & 104 & $-1(107)$ & 0.990 & 0.000 \\
Time trend (years) & 104 & $154(107)$ & 0.156 & 0.010 \\
Outcome (target pregnancy) & & & & \\
Recurrence of PE/HELLP (0=no; 1=yes) & 92 & $3720(967)$ & $0.000^{*}$ & 0.141 \\
Gestational age at delivery (weeks) & 104 & $-290(88)$ & $0.002^{*}$ & 0.094 \\
\hline Child characteristics & & & & \\
Baseline (index pregnancy) & & & & \\
SGA (0=no; 1=yes) & 104 & $-1195(799)$ & 0.138 & 0.021 \\
Outcome (target pregnancy) & & & & \\
Birth weight (g) & 104 & $-1(0)$ & $0.003^{*}$ & 0.086 \\
IUFD (0=no; 1=yes) & 103 & -1952 (1776) & 0.274 & 0.012 \\
\hline
\end{tabular}

*Significant results ( $\alpha$ was set at 0.10 )

Beta coefficients and their SEs are rounded

In a backward multivariate regression analysis, after adjustment for all other factors, only the development of recurrent PE/HELLP ( $\mathrm{B}=3332 ; p<0.0001)$, the gestational age at delivery of the target pregnancy $(B=-240 ; p=0.010)$ and the occurrence of IUFD ( $B=-$ 4803; $p=0.005$ ) were independent predictors of maternal hospital costs. Together, 
these variables explained $26 \%$ of the hospital costs $\left(R^{2}\right)$. Only outcome variables are associated with maternal hospital costs.

We repeated these univariate and multivariate regression analyses in the subgroup of women who did not develop recurrent PE/HELLP ( $n=76$ out of 104). Gestational age at delivery (target pregnancy) and birth weight did no longer contribute to the relation in the univariate analyses (Table 6).

In the backward multivariate analysis in this subgroup, only maternal BMI was an independent predictor of maternal hospital costs $(B=-133 ; p=0.059)$. About $4.8 \%$ of the costs $\left(R^{2}\right)$ were explained by BMI.

Table 6: Univariate regression analyses examining the relation between mother and child characteristics and maternal hospital costs, including only women without a recurrence PE/HELLP $(n=76)$

\begin{tabular}{lllll}
\hline Variable & $\mathrm{n}$ & $\mathrm{B}(\mathrm{SE})$ & $p$ & $\mathrm{R}^{2}$ \\
\hline Maternal characteristics & & & & \\
Baseline (before and index pregnancy) & 76 & $189(74)$ & $0.012^{*}$ & 0.082 \\
Age at conception (years) & 76 & $905(423)$ & $0.036^{*}$ & 0.058 \\
Gravidity & 76 & $2421(736)$ & $0.002^{*}$ & 0.128 \\
Parity & 76 & $-128(78)$ & 0.105 & 0.035 \\
BMI & 76 & $-29(108)$ & 0.790 & 0.001 \\
Gestational age delivery index pregnancy (weeks) & 76 & $119(101)$ & 0.240 & 0.005 \\
Time trend (years) & & & & \\
Outcome (target pregnancy) & 76 & $-112(101)$ & 0.273 & 0.016 \\
Gestational age at delivery (weeks) & & & & \\
\hline Child characteristics & & & & \\
Baseline (index pregnancy) & 76 & $-119(800)$ & 0.882 & 0.000 \\
SGA (0=no; 1=yes) & 76 & $-826(1808)$ & 0.649 & 0.003 \\
Outcome (target pregnancy) & & &
\end{tabular}

*Significant results ( $\alpha$ was set at 0.10)

Beta coefficients and their SEs are rounded

\section{Comments}

This study explored the hospital cost drivers in relation to maternal and child outcome of 104 next pregnancies in women with a history of early-onset PE/HELLP, managed according to CAU. Maternal costs per patient averaged $€ 8,047$. As expected, the most important contributors to hospital costs were maternal admissions and outpatient visits, which together comprised over $80 \%$ of the total maternal costs. Costs associated 
with hospital neonatal care were excluded as they are not part of the care provided by gynecologists/obstetricians. We did not find similar studies on costs of 'uneventful' pregnancies or guidelines about how to standardize the care in next pregnancies in women with a history of early-onset PE/HELLP.

This study focuses on the wide variation in CAU for pregnant women with a history of PE or HELLP, caused among others, by the wide range (5 to 37) of number of outpatient visits. The variation in maternal hospital costs was not explained by the calendar year of pregnancy (period 1996-2002). Maternal hospital costs in these women were particularly high when recurrent PE/HELLP had developed or when pregnancy led to preterm birth. Although multiple regression analyses indicated that these complications in the target pregnancy had an important impact on maternal hospital costs, over $70 \%$ of the variation in maternal hospital costs could not be accounted for by all factors included in our regression models, i.e. baseline characteristics of the participants and maternal and child outcome. An additional multiple regression analysis, including only the larger subgroup of women who did not develop recurrent PE/HELLP, showed that only BMI was an independent predictor of maternal hospital costs. In fact, all outcome variables that contributed to maternal hospital costs in the previous multivariate regression analysis were related to PE/HELLP recurrence. It is conceivable that other mostly subjective factors contributed to the costs (such as patient preferences and doctor's practice style) (16). These factors are not necessary superfluous as they may have improved patient's satisfaction, a yield not quantifiable in our study. These inferences indicate that clinical and outpatient care provided to this specific cohort of pregnant women and with it, the cost-effectiveness, can be improved by standardization of care $(17,18)$. Therefore, we propose a standardized management protocol based on recurrence risk stratification. This method is one of the possibilities, which can be expected to reduce the number of superfluous outpatient visits and diagnostic procedures.

\section{Strengths and limitations}

This is the first study reporting on the variability in patterns of care (and associated costs) provided to pregnant women with a history of early-onset PE/HELLP in the Netherlands, a form of care hampered by the lack of a standardized management protocol.

The results of this study indicate that the care for this population is not costeffective. One important quality aspect of this study was our standardized procedures to retrieve data from medical records. The data were collected within the wellorganized structure of the Dutch Obstetric Consortium (19). Obviously, our study has also a number of limitations. First, inherent to its retrospective nature, some variables 
may have been underestimated because of their incomplete registration in the medical records (information bias). Only few sociodemographic data were registered. Previous studies showed that for example educational level, ethnicity and income are factors that affect health care consumption $(20,21)$. Such sociodemographic information can help in the development of optimal intervention strategies and appropriate health policies. Another aspect is that most of the data in this study were obtained in university hospitals. However, in the Netherlands, most women with a history of earlyonset PE/HELLP will get their prenatal care in the next pregnancy in university hospitals. It should be noted that differences in the risk level of the recruited local patient populations, in the absence of a generally accepted guideline, can be expected to influence intensity and content of the care offered in each participating hospital and with it, reduce the inter-hospital comparability. We consider this study a first step towards the development of cost-effective management in the next pregnancy of women, who experienced early-onset PE/HELLP in their previous pregnancy.

\section{References}

1. Isler CM, Rinehart BK, Terrone DA, May WL, Magann EF, Martin JN, Jr. The importance of parity to major maternal morbidity in the eclamptic mother with HELLP syndrome. Hypertension in pregnancy : official journal of the International Society for the Study of Hypertension in Pregnancy. 2003;22(3):287-94.

2. Hernandez-Diaz S, Toh S, Cnattingius S. Risk of pre-eclampsia in first and subsequent pregnancies: prospective cohort study. BMJ. 2009;338:b2255.

3. Andersgaard AB, Acharya G, Mathiesen EB, Johnsen SH, Straume B, Oian P. Recurrence and long-term maternal health risks of hypertensive disorders of pregnancy: a population-based study. American journal of obstetrics and gynecology. 2012 Feb;206(2):143 e1-8.

4. Grimshaw JM, Hutchinson A. Clinical practice guidelines--do they enhance value for money in health care? British medical bulletin. 1995 Oct;51(4):927-40.

5. Grimshaw JM, Russell IT. Effect of clinical guidelines on medical practice: a systematic review of rigorous evaluations. Lancet. 1993 Nov 27;342(8883):1317-22.

6. Wollersheim H, Burgers J, Grol R. Clinical guidelines to improve patient care. The Netherlands journal of medicine. 2005 Jun;63(6):188-92.

7. Fernandez-Olano C, Hidalgo JD, Cerda-Diaz R, Requena-Gallego M, Sanchez-Castano C, UrbistondoCascales $\mathrm{L}$, et al. Factors associated with health care utilization by the elderly in a public health care system. Health Policy. 2006 Jan;75(2):131-9.

8. Abram K, Silm H, Maaroos HI, Oona M. Subjective disease perception and symptoms of depression in relation to healthcare-seeking behaviour in patients with rosacea. Acta dermato-venereologica. 2009;89(5):488-91.

9. Thompson BT, Schoenfeld D. Usual care as the control group in clinical trials of nonpharmacologic interventions. Proceedings of the American Thoracic Society. 2007 Oct 1;4(7):577-82.

10. Delahaije DH, van Kuijk SM, Dirksen CD, Sep SJ, Peeters LL, Spaanderman ME, et al. Cost-effectiveness of recurrence risk guided care versus care as usual in women who suffered from early-onset preeclampsia including HELLP syndrome in their previous pregnancy (the PreCare study). BMC pregnancy and childbirth. 2010;10:60. 
11. van Kuijk SM, Nijdam ME, Janssen KJ, Sep SJ, Peeters LL, Delahaije DH, et al. A model for preconceptional prediction of recurrent early-onset preeclampsia: derivation and internal validation. Reprod Sci. 2011 Nov;18(11):1154-9.

12. CLASP: a randomised trial of low-dose aspirin for the prevention and treatment of pre-eclampsia among 9364 pregnant women. CLASP (Collaborative Low-dose Aspirin Study in Pregnancy) Collaborative Group. Lancet. 1994 Mar 12;343(8898):619-29.

13. Oostenbrink J, Bouwmans C, Koopmanschap M, Rutten FFH. Handleiding voor kostenonderzoek, methoden en standaard kostprijzen voor economische evaluaties in de gezondheidszorg. Diemen: College voor Zorgverzekeringen; 2004.

14. Netherlands S. StatLine Den Haag: Statistics Netherlands; 2010. Available from: http://www.statline.cbs.nl.

15. Donders AR, van der Heijden GJ, Stijnen T, Moons KG. Review: a gentle introduction to imputation of missing values. Journal of clinical epidemiology. 2006 Oct;59(10):1087-91.

16. Birkmeyer JD, Reames BN, McCulloch P, Carr AJ, Campbell WB, Wennberg JE. Understanding of regional variation in the use of surgery. Lancet. 2013 Sep 28;382(9898):1121-9.

17. Gutierrez IM, Zurakowski D, Chen Q, Mooney DP. Clinical practice guidelines (CPGs) reduce costs in the management of isolated splenic injuries at pediatric trauma centers. Langenbeck's archives of surgery / Deutsche Gesellschaft fur Chirurgie. 2013 Feb;398(2):313-5.

18. Panella M, Marchisio S, Di Stanislao F. Reducing clinical variations with clinical pathways: do pathways work? Int J Qual Health Care. 2003 Dec;15(6):509-21.

19. Dutch Obstetrics Consortium 2009. Available from: www.studies.obsgyn.nl.

20. Celik Y, Hotchkiss DR. The socio-economic determinants of maternal health care utilization in Turkey. Soc Sci Med. 2000 Jun;50(12):1797-806.

21. Weiss JE, Greenlick MR. Determinants of medical care utilization: the effect of social class and distance on ontacts with the medical care system. Medical care. 1970 Nov-Dec;8(6):456-62. 


\section{CHAPTER 4}

Cost-effectiveness of recurrence risk guided

care in the next pregnancy of women who

experienced early-onset preeclampsia or HELLP

syndrome in their previous pregnancy

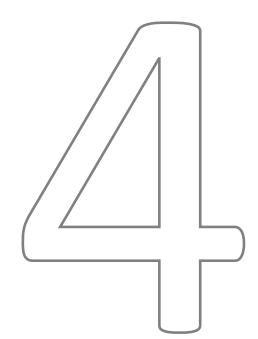

Denise HJ Delahaije, Sander MJ van Kuijk, Louis L Peeters, Liesbeth CJ Scheepers, Marc E Spaanderman, Johannes J Duvekot, Mariëlle G van Pampus, Wessel J Ganzevoort, Thea KI van Asselt, Luc J Smits, Carmen D Dirksen for the PreCare study group 


\begin{abstract}
Objective

To compare the cost-effectiveness of recurrence risk guided care with care-as-usual in the next pregnancy provided to women whose preceding pregnancy was complicated by early-onset preeclampsia or the HELLP syndrome.
\end{abstract}

\title{
Study design
}

We conducted a before-after study in six university and six non-university hospitals in the Netherlands. A total of 311 newly pregnant, former patients were included. We implemented a model to predict recurrent early-onset preeclampsia or HELLP and compared recurrence risk guided care with care-as-usual with respect to costs, effectiveness and cost-effectiveness. Two incremental cost-effectiveness ratios (ICERs) were calculated expressing 1) the hospital perspective reflected in cost per prevented adverse neonatal outcome, and 2) the societal perspective reflected in cost per Quality Adjusted Life Years (QALY).

\section{Results}

From the hospital perspective, recurrence risk guided care was less costly (costs $€ 11,400$ vs. $€ 13,755$; not significant) than care-as-usual, with a comparable rate of adverse neonatal outcome (0.90 vs. 0.89 ; not significant), resulting in a more favorable cost-effectiveness for recurrence risk guided care. In contrast, from the societal perspective, recurrence risk guided care was both more costly than care-as-usual (costs $€ 35,283$ vs. €32,729; not significant) and less effective in terms of QALYs (0.75 vs. 0.81; not significant).

\section{Conclusions}

From a hospital perspective, the impact on costs of implementing recurrence risk guided care in the next pregnancy to formerly preeclamptic patients is modest. However, from the societal perspective, recurrence risk guided care is associated with a shift from hospital costs to health care costs outside the hospital while also reducing healthrelated quality of life. 


\section{Introduction}

Preeclampsia (PE) and the HELLP syndrome affect 2 to $7 \%$ of all pregnancies in nulliparous women (1). These disorders may have long-term consequences for mother and child $(2,3)$. Particularly women with a history of PE/HELLP are at increased risk of recurrence in their next pregnancy (4). There is no consensus about the clinical management in the next pregnancy. As a result, follow-up and counseling vary widely per country, center, gynecologist and patient, with care largely determined by subjective criteria, and thus possibly not cost-effective. Since only part of these women develop recurrent disease in their next pregnancy, the management in that pregnancy may benefit from differentiating between those at low and at high recurrence risk. We developed a prediction model that enables tailoring the intensity of care to each patient's estimated recurrence risk (5). Particularly low-risk patients are likely to benefit from this type of risk stratification, by averting superfluous surveillance and associated usage of health care resources. It follows that recurrence risk guided care (RGC) has the potential to reduce the costs of care without compromising pregnancy outcomes and quality of life.

This study was designed to explore whether RGC was more cost-effective than care-asusual (CAU) in the clinical management of these women in their next pregnancy. To this end, we developed a model to predict recurrent early-onset PE/HELLP and compared the cost-effectiveness of RGC with that of CAU in 311 newly pregnant former patients.

\section{Materials and methods}

This study is part of the so-called "PreCare study" which has been detailed elsewhere (6). The study consisted of two phases. In the first phase, we followed CAU patients from their first outpatient visit in pregnancy until 3 months postpartum. An additional group had been also managed by CAU in the past. We retrieved the (anonymized) data from this group retrospectively from the hospital information system. In the second phase, we introduced our prediction model into clinical practice.

The patient population consisted of pregnant women over 18 years of age, who had developed early-onset (<34 weeks) PE/ HELLP in their previous pregnancy and had their first consultation at the outpatient clinic before the 20th week of pregnancy. We excluded women with severe co-morbidity (diabetes mellitus, systemic lupus erythematosus, renal disease, cardiac disease and the anti-phospholipid syndrome) or who had delivered at term ( $>37$ weeks) in their previous pregnancy. The study was ap- 
proved by the Medical Ethical Review Board of the Maastricht University. We matched each enrolled patient in RGC to a patient receiving CAU patient for age category (10year age groups: $20-30 ; 31-40 ; 41-50)$, center and pregnancy length in the previous pregnancy (<34 weeks and between 34 and 37 weeks).

In $\mathrm{CAU}$, obstetricians managed their patients in whatever manner they deemed appropriate. RGC consisted of two monitoring protocols. The Medium Care (MC) and High Care (HC) protocol contained 9-11 and 14-16 outpatient visits, respectively. Patients allocated to $\mathrm{HC}$ were allowed to undergo additional diagnostic tests. Activities in addition to those described in the protocols were registered.

We performed an economic evaluation with a time horizon of one year. We calculated two incremental cost-effectiveness ratios (ICERs) expressing 1) hospital cost per prevented adverse neonatal outcome (child outcome), from the hospital perspective, and 2) societal cost per Quality Adjusted Life Year, i.e. QALY (maternal outcome), from the societal perspective. An adverse outcome was defined as an admission to the neonatal intensive care unit (NICU) or perinatal mortality. If a newborn required NICU admission for at least one day or died, this was coded as 0 (i.e. a negative outcome); in all other cases, the outcome was coded as 1 (i.e. a positive outcome). The difference in proportions of positive outcome reflects the number of adverse cases prevented. For calculation of the ICER, we subtracted the costs of a NICU admission from the total costs to avoid double-counting.

QALYs were estimated using a generic health-related quality of life instrument, the EuroQol (EQ-5D) (7). For each health state, a utility score can be computed, based on preferences from a general Dutch population (8). The EQ-5D was sent together with the cost questionnaires to all women followed prospectively. Cost analyses were performed according to the Dutch guideline (9). Costs were expressed in 2011 Euros. If necessary, costs prices were actualized to 2011 using the Dutch Consumer Price Index (10). Health care-related unit prices were obtained from published guidelines $(9,11)$. Costs per patient were calculated by multiplying volumes with unit prices of the resources used (9).

The analysis from the hospital perspective only included costs made within the hospital. The data on individual hospital resource use were retrieved from the hospital information systems. The analysis from the societal perspective also included costs outside the hospital by means of retrospective cost questionnaires with a recall period of 3 months. The costs of prescription drugs used by the participants were obtained from the Dutch pharmacotherapeutic compass (12). Productivity costs due to sick leave were calculated using the friction costs method (11). 


\section{Statistical analysis}

Analyses were performed based on intention to treat. The dataset was checked for completeness and validity by consultation of the research nurses in the hospitals in case of inconsistencies. We employed single imputation to replace missing values (13), followed by bootstrapping to determine the 95\% confidence intervals around costdifferences between RGC and CAU and to quantify the degree of uncertainty around the ICER. Based on bootstrapped cost-effectiveness results (data not shown), costeffectiveness acceptability curves were constructed, showing the probability that RGC is cost-effective using a range of ceiling ratios (14). Bootstrapping was performed using Excel 2010. Other analyses were performed using SPSS, version 20.0 for Windows (SPSS INC 2012). Additionally, subgroup and sensitivity analyses were performed. For the hospital perspective, we evaluated the effect on the cost-effectiveness ratio of age ( $<$ or $\geq$ the median), the gestational age at birth in the previous pregnancy ( $<34$ weeks or between 34 and 37 weeks) and whether the previous pregnancy resulted in the birth of a small-for-gestational-age (SGA) infant. For the societal perspective, we also performed analyses for a low vs. high anxiety group (< or $\geq$ the median), a low vs. high depression group ( $<$ or $\geq$ the median) and for signs vs. no signs of posttraumatic stress disorder at baseline ( $<$ or $\geq$ the median). Besides, differences between RGC and CAU may partly result from differences in unit prices per admission day between university and non-university hospitals. In the analysis from the societal perspective, 93\% (CAU) and $84 \%$ (RGC) of the admissions were in university hospitals. This potential effect was tested by applying the same unit prices irrespective of type of hospital. In the analysis from the hospital perspective, about the same proportions of admissions in CAU and RGC were in a university hospital. Therefore, we refrained from this sensitivity analysis in the analysis from the hospital perspective. An imbalance in baseline utility scores between RGC and CAU can result in a misleading incremental cost-effectiveness ratio. Therefore, we adapted the utility scores in one group by correcting for the baseline difference between the two groups (15).

\section{Results}

\section{Patient population}

In the analysis from the hospital perspective, we included 311 patients (152 CAU and $159 \mathrm{RGC}, 37$ receiving $\mathrm{HC}$ and $122 \mathrm{MC}$ ). For the analysis from the societal perspective, only prospectively included patients were considered $(n=207)$. Of these, 185 had returned at least one questionnaire and were included in the analyses (45 CAU and 140 
RGC, 34 receiving $\mathrm{HC}$ and $106 \mathrm{MC}$ ). Baseline characteristics of the 185 women are presented in Table 1.

Table 1: Characteristics of the selection of women for the cost-effectiveness analysis

\begin{tabular}{|c|c|c|c|c|c|}
\hline & $\begin{array}{l}\text { Recurrence risk } \\
\text { guided care }^{1,2} \\
(n=159)\end{array}$ & $\begin{array}{l}\text { Care-as-usual } \\
\text { total }^{1} \\
(n=152)\end{array}$ & $\begin{array}{l}\text { Care-as-usual } \\
\text { prospectively } \\
\text { included }^{1,2} \\
(n=48)\end{array}$ & $\begin{array}{l}\text { Care-as-usual } \\
\text { retrospectively } \\
\text { included }^{1} \\
(n=104)\end{array}$ & $\mathrm{p}$-value \\
\hline \multicolumn{6}{|l|}{ Maternal characteristics } \\
\hline Maternal age at conception (y) & $30.92(4.44)$ & $31.14(5.01)$ & $32.37(5.26)$ & $30.57(4.81)$ & 0.68 \\
\hline Body mass index $\left(\mathrm{kg} / \mathrm{m}^{2}\right)$ & $25.58(6.01)$ & $26.33(5.58)$ & $25.33(4.88)$ & $27.26(6.06)$ & 0.32 \\
\hline Gravidity & $2.52(0.84)$ & $2.59(1.35)$ & $2.96(1.98)$ & $2.42(0.88)$ & 0.56 \\
\hline Parity & $1.11(0.38)$ & $1.15(0.57)$ & $1.17(0.75)$ & $1.15(0.47)$ & 0.41 \\
\hline \multicolumn{6}{|l|}{ Treated in } \\
\hline University hospital, n (\%) & $135(84.9)$ & $132(86.8)$ & $45(93.8)$ & $87(83.7)$ & 0.62 \\
\hline Non-university hospital, n (\%) & $24(15.1)$ & $20(13.2)$ & $3(6.3)$ & $17(16.3)$ & \\
\hline \multicolumn{6}{|c|}{ Characteristics of previous pregnancy } \\
\hline Gestational age (weeks) & $31.12(3.79)$ & $31.11(3.56)$ & $31.18(3.12)$ & $31.08(3.75)$ & 0.99 \\
\hline Birthweight (g) & $1409.29(729.49)$ & $1432.55(734.49)$ & $1404.02(716.48)$ & $1467.79(764.53)$ & 0.82 \\
\hline Low birthweight (<2500 g) & $130(81.76)$ & $70(46.05)$ & 38 (79.17) & $32(30.77)$ & \\
\hline Very low birthweight (<1500 g) & $92(57.86)$ & $45(29.61)$ & $28(58.33)$ & $17(16.35)$ & \\
\hline
\end{tabular}

Data are given as mean \pm SD or as percentages

1) Hospital perspective

2) Societal perspective

\section{Hospital perspective}

Our analyses showed that RGC was not associated with a higher rate of adverse neonatal outcome, indicated by comparable proportions of prevented adverse neonatal outcome in the subgroups receiving RGC (90\%) and CAU (89\%). In RGC, the rate of adverse neonatal outcomes was slightly higher in $\mathrm{HC}(14 \%)$ than in $\mathrm{MC}(9 \%)$.

Mean hospital costs per patient did not differ significantly between women receiving RGC and those receiving CAU (mean difference €305; 95\% Cl from -€4,960 to $€ 4,456)$. Neither maternal outpatient visits (16 and 17) nor admission days (7 and 8) differed appreciably between RGC and CAU (Table 2). The same applied to the total hospital costs for the mother (mean difference $€ 468 ; 95 \% \mathrm{Cl}$ from - $€ 1,413$ to $€ 532$ ) and the child (mean difference $€ 163 ; 95 \% \mathrm{Cl}$ from -€4,024 to €4,532). Infant costs in RGCHC $(€ 13,016)$ were almost twice as high as those in RGC-MC $(€ 6,389)$, without an appreciable difference in maternal costs ( $H C € 7,211$ and $M C € 7,638$ ). 
For calculation of the ICER, we subtracted the costs associated with NICU admissions from total hospital costs, resulting in mean hospital costs of $€ 11,400$ for RGC vs. $€ 13,755$ for CAU. Calculation of the point estimate of the ICER was not needed as RGC is dominant over CAU (less costly and more effective). See Table 4.

The cost-effectiveness acceptability curve illustrates an $80-95 \%$ probability of RGC being more cost-effective than CAU (Figure 1). 


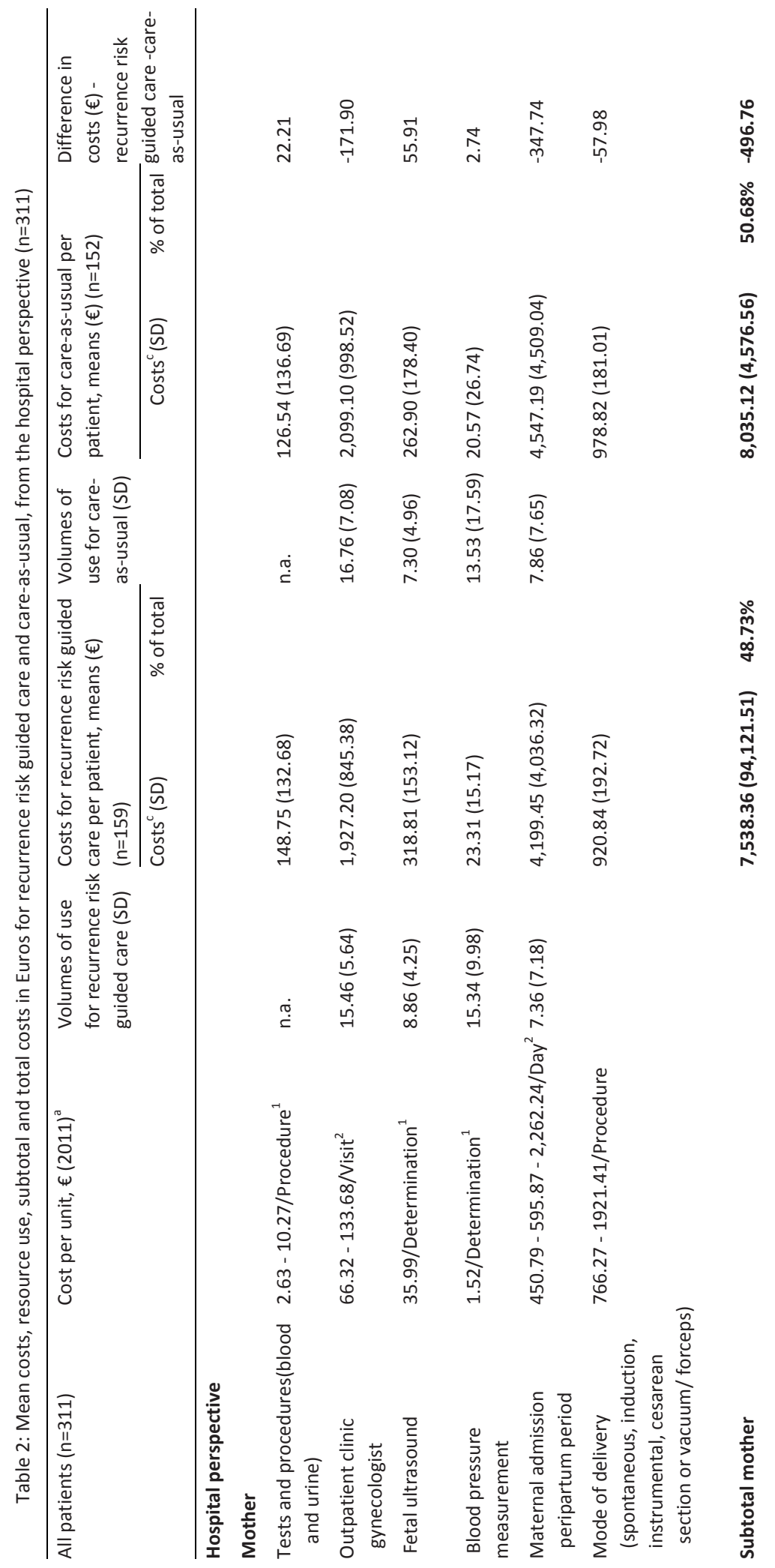




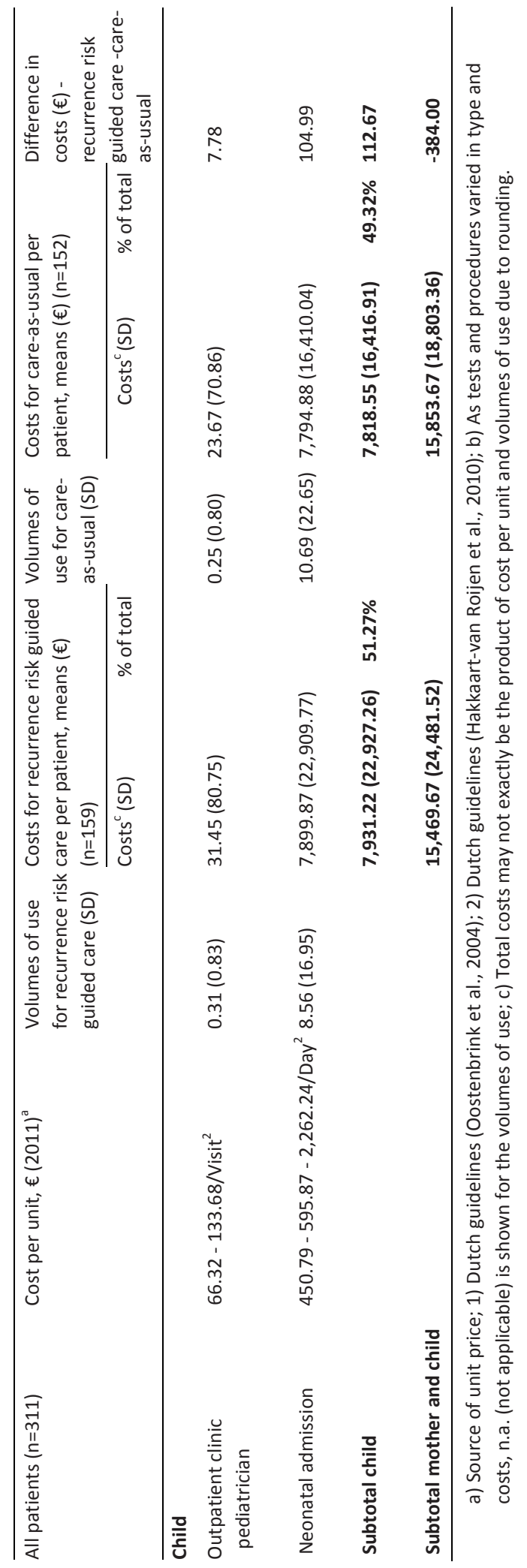




\section{Cost-effectiveness acceptability curve}

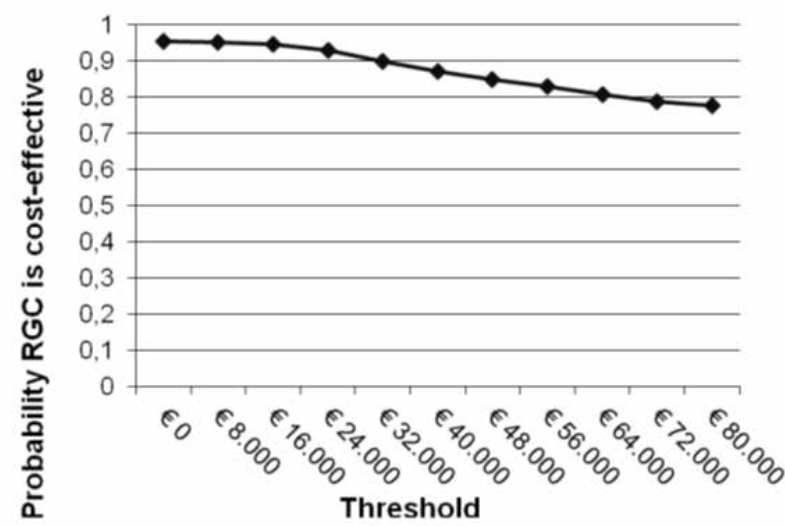

Figure 1: Cost-effectiveness acceptability curve: costs per adverse outcome child prevented

\section{Societal perspective}

The utility scores at baseline and at follow-up were slightly higher in CAU than in RGC (0.03), resulting in lower QALYs in RGC than in CAU (0.75 and 0.81 , respectively). In RGC, HC patients reported consistently higher utilities than MC patients at all measurement moments resulting in higher QALYs (0.78 and 0.75 respectively).

The mean societal costs per patient in RGC were not significantly different from the costs per patient in CAU (bootstrap mean difference $€ 2,722 ; 95 \% \mathrm{Cl}$ from -€4,968 to $€ 9,993$ ). Taking only hospital costs into account, RGC differed from CAU by resulting in less total costs for mother and child (Table 3). Conversely, RGC tended to be associated with higher total costs outside the hospital than CAU $(€ 2,651 ; 95 \% \mathrm{Cl}$ from $€ 1,463$ to $€ 6,361$ ), of which the health care component was significantly different (bootstrap mean difference $€ 1,799 ; 95 \% \mathrm{Cl}$ from $€ 527$ to $€ 2,973$ ). In RGC, hospital costs for $\mathrm{HC}$ and $\mathrm{MC}$ were $€ 21,180$ and $€ 14,017$ respectively, with health care costs outside the hospital being $€ 5,003$ and $€ 5,574$. Calculation of the point estimate of the ICER from the societal perspective was not necessary as RGC is inferior to CAU (i.e. more costly and less effective).

Irrespective of the ceiling ratio, RGC has a low probability of being cost-effective, ranging from $30 \%$ to $5 \%$ (Figure 2 ). These results indicate that, from the societal perspective, RGC is not cost-effective. 


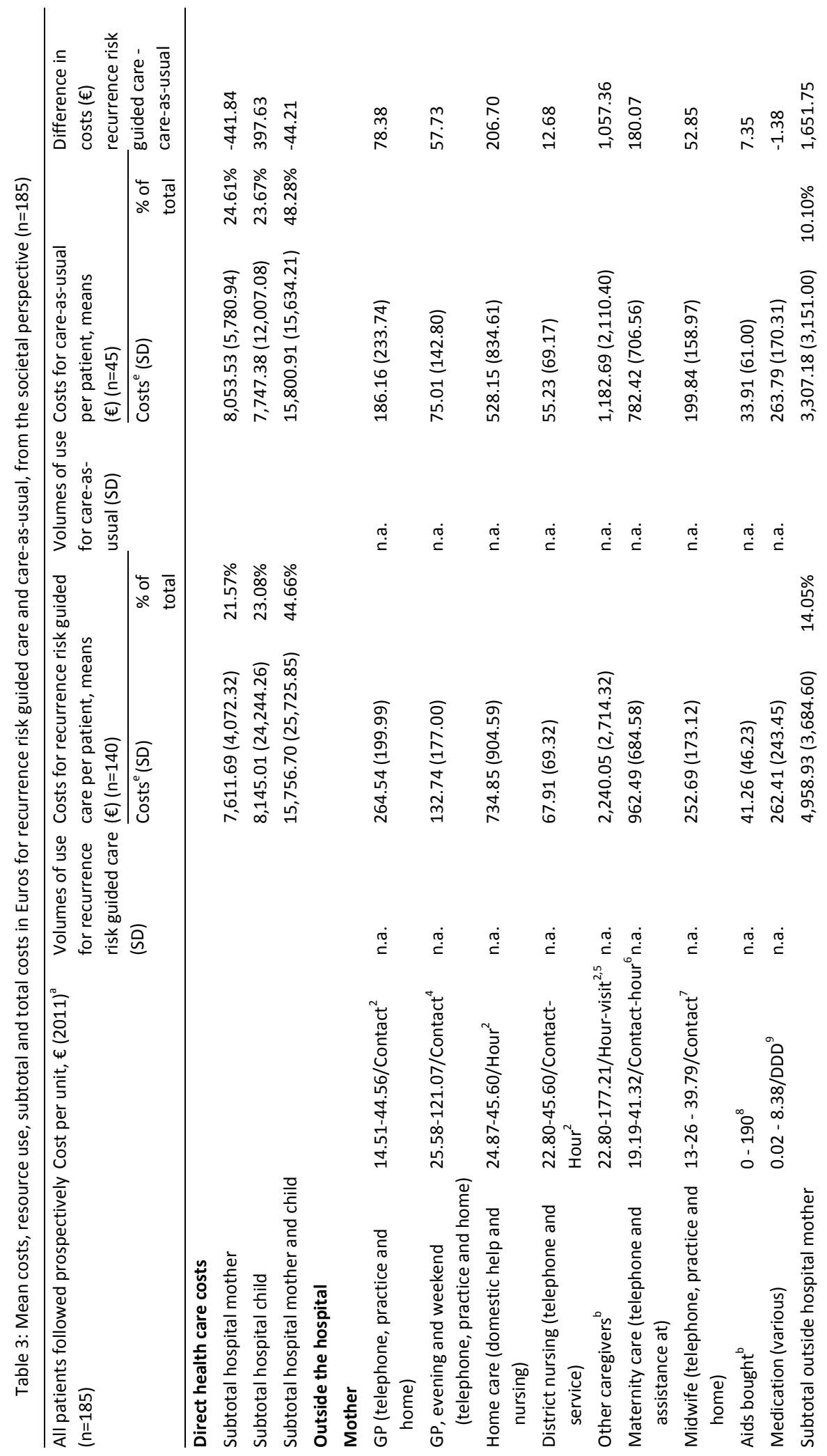




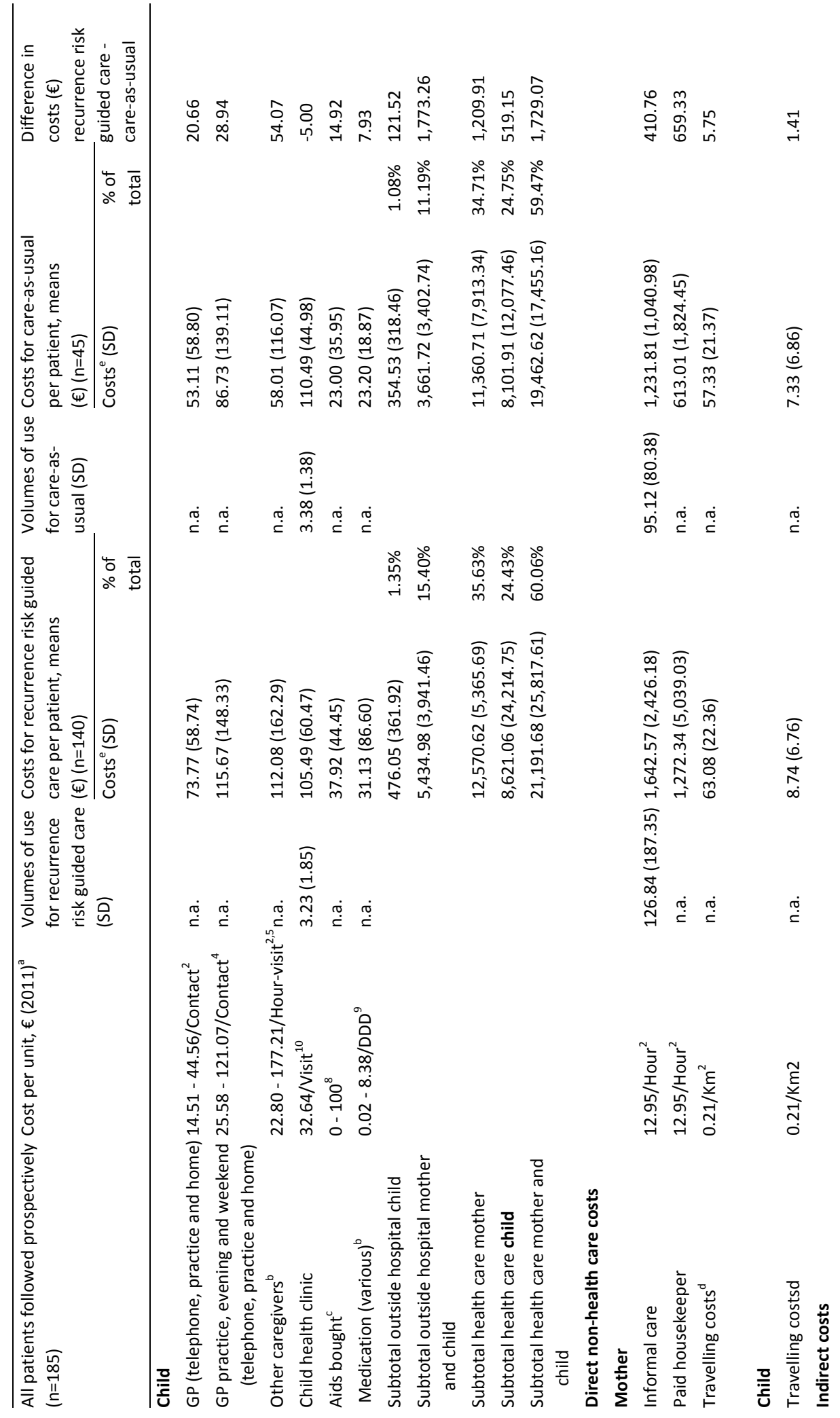




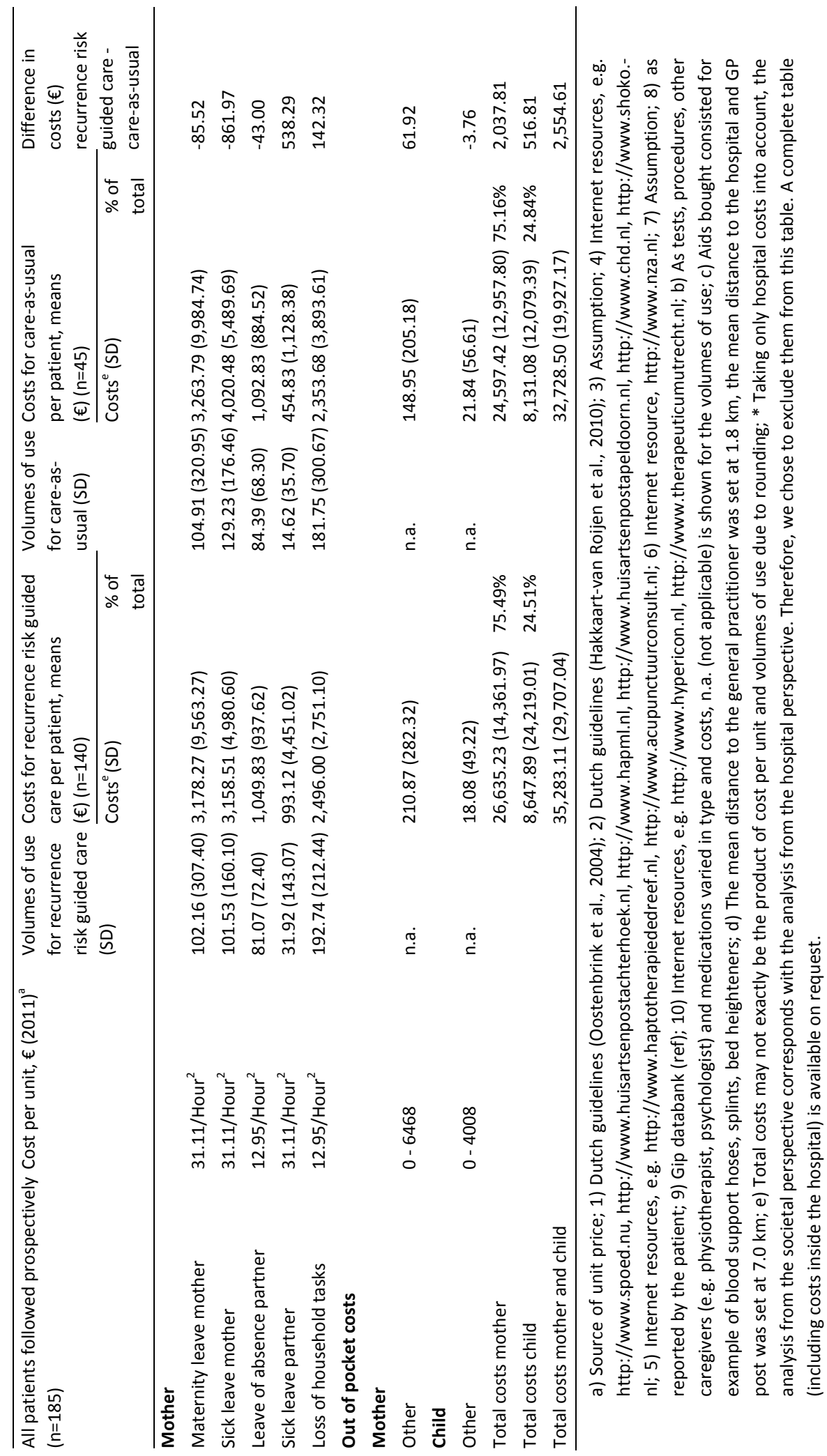




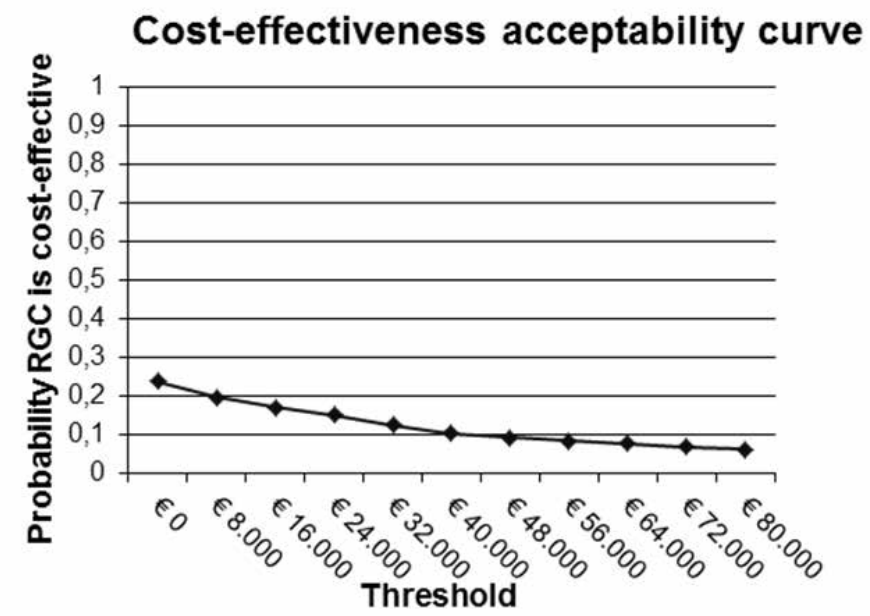

Figure 2: Cost-effectiveness acceptability curve: costs per QALY

Subgroup analyses from the hospital perspective (Table 4) revealed that RGC was more cost-effective in older women, who in their previous pregnancy had given birth after the $34^{\text {th }}$ week to a non-SGA infant. For almost all other subgroups, RGC was less costly, though also slightly less effective. Subgroup analyses from the societal perspective indicated that, for most subgroups, RGC was less cost-effective than CAU. Exclusion of an outlier (extremely high neonatal costs, i.e. $€ 221,070$ ), applying the same unit prices to university and non-university hospitals and using the mean difference adjustment method for baseline utilities did not change our conclusion about RGC being more costly than CAU. Overall, subgroup and sensitivity analyses show a clear trend towards more costs and lower QALYs in patients receiving RGC. 


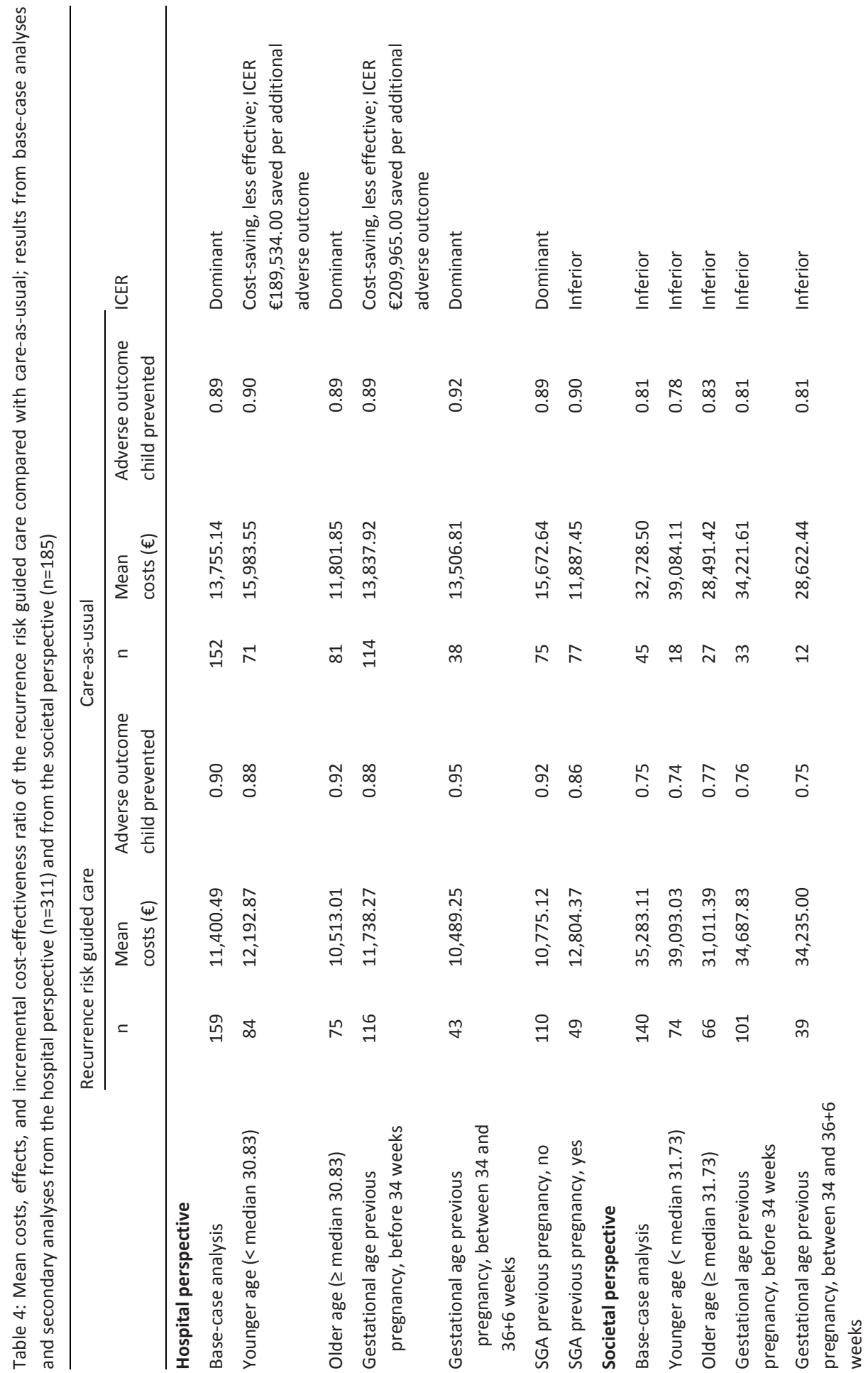




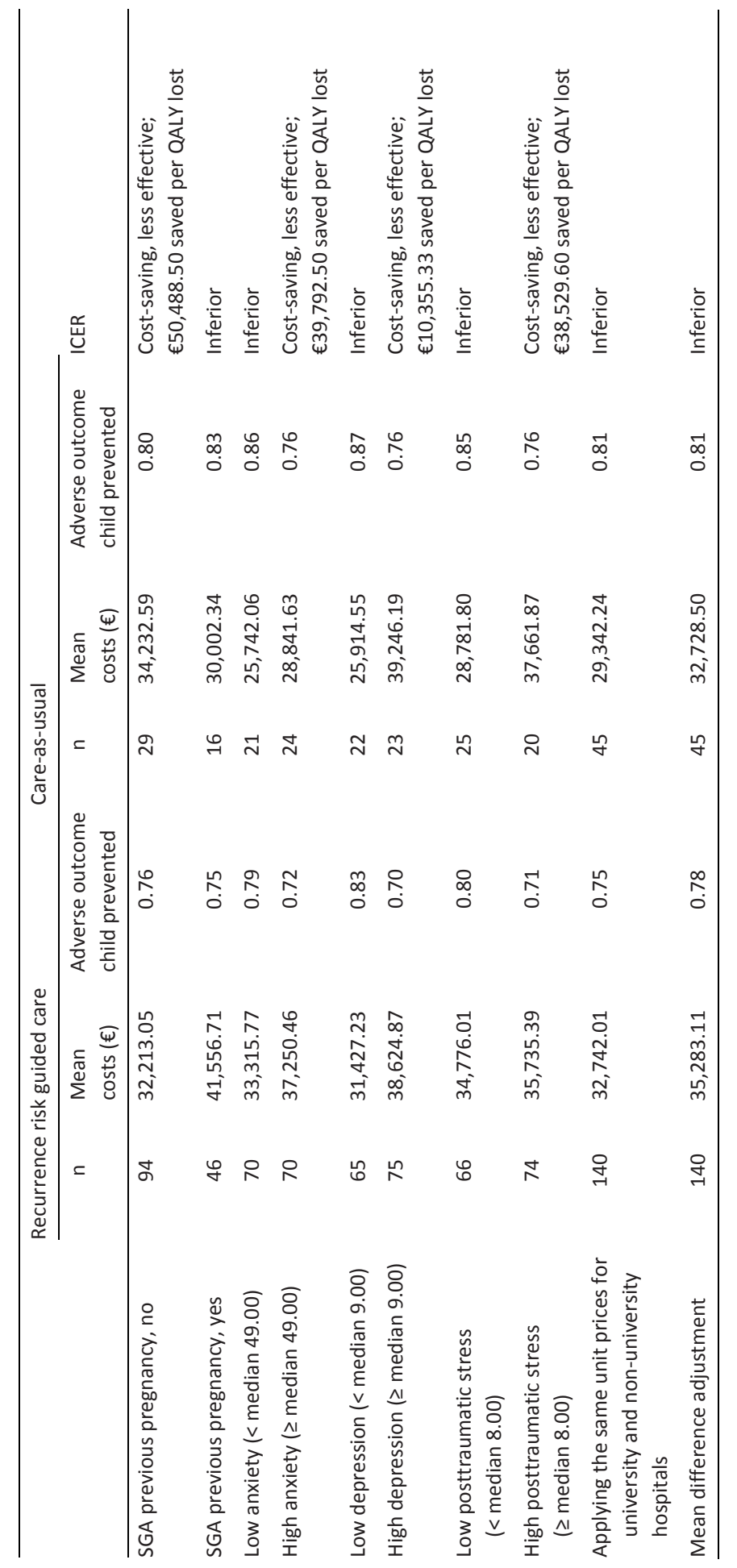




\section{Comment}

This study was designed to investigate whether RGC is more cost-effective than CAU in the next pregnancy of women whose previous pregnancy had been complicated by early-onset PE/HELLP.

The base-case results support the concept that RGC leads to lower maternal hospital-related costs and thus, is more cost-effective than CAU from a hospital perspective. Conversely, from the societal perspective, RGC was less cost-effective than CAU, due to lower reported quality of life and higher costs outside the hospital. The latter seemed to result from patients in the MC subgroup, visiting more often other care providers than CAU patients. Utilities were consistently higher in CAU than in RGC at all measurement moments, contributing to higher QALYs in CAU. In the RGC group, both $\mathrm{HC}$ and MC subgroups reported lower utilities than CAU at all four measurement moments. Bootstrap results showed that RGC was less effective in terms of QALYs $(99 \%$ of replications), and led to higher societal costs.

To our knowledge, this study is the first that evaluates the economic effects of recurrence risk-guided interventions in pregnant women who experienced PE/HELLP in their previous pregnancy. Multi-parameter tools for the prediction of recurrent earlyonset PE/HELLP have only been reported recently (16). Besides, no evidence-based guidelines for the management of this particular group of former patients are available. The results of the PreCare study were disappointing. Our prediction model discriminated poorly (17). Nevertheless, implementing the model into clinical practice had no appreciable effect on the maternal and perinatal outcome (18). Adherence to the $\mathrm{HC}$ and $\mathrm{MC}$ protocols was disappointing, as reflected in the wide variation in the number of visits to the outpatient clinic in the RGC group. The intensity of the clinical management in these patients is influenced by the experience and expertise of the obstetrician, and confidence of the patient in herself and her doctor. Besides, also the unpredictability in clinical presentation of PE/HELLP plays a role, as it may vary widely in severity and rate of deterioration. We explored several patient related factors (i.e. recurrence of PE/HELLP, depression, anxiety, posttraumatic stress, QALY, the level of satisfaction) and could not detect a relation with the (excess) number of outpatient visits (data not shown). Nevertheless, cost-effectiveness analyses from both perspectives confirm that hospital costs were lower in RGC than in CAU. Particularly the costs outside the hospital boost the total costs for RGC relative to those for CAU. This may be related to the (perceived) lower allowance for outpatient visits in RGC driving them to seek support and/or reassurance from alternative caregivers. This may also have caused the lower health-related quality of life in RGC compared to CAU. This problem may be solved by creating an easy-accessible back-up system for patients, consisting of a same-day-call-back-service, e.g. by specialized nurse practitioners. 
The study had various limitations. Some variables had missing values. These variables consisted of predictor variables, indicators of intensity of care, indicators of costeffectiveness and, in some cases, important outcome variables. Omitting patients with one or more missing variables from the analysis would have reduced statistical power markedly, potentially introducing selection bias. In the original PreCare dataset we measured a wide range of variables that were included in the imputation model, increasing the likelihood of valid predictions of replacements for missing values. Therefore, we are confident that the regression imputation we used adds to the accuracy of our results. Finally, the datasets available for data retrieval from retrospectively assessed patients in the CAU group were often incomplete and/or contained errors. Some variables may have been underestimated because of incomplete registration in the medical records (information bias). In addition, because they were included retrospectively, these women could not fill out questionnaires.

We conclude that, from a hospital perspective, it would be cost-effective to offer previous preeclamptic women RGC instead of CAU. However, from a societal perspective, RGC is not cost-effective, which is due to a lower health-related quality of life and a shift to health care consumption outside the hospital.

\section{References}

1. Sibai B, Dekker G, Kupferminc M. Pre-eclampsia. Lancet. 2005 Feb 26-Mar 4;365(9461):785-99.

2. Duley L. The global impact of pre-eclampsia and eclampsia. Seminars in perinatology. 2009 Jun;33(3):130-7.

3. Ghulmiyyah L, Sibai B. Maternal mortality from preeclampsia/eclampsia. Seminars in perinatology. 2012 Feb;36(1):56-9.

4. Barton JR, Sibai BM. Prediction and prevention of recurrent preeclampsia. Obstetrics and gynecology. 2008 Aug;112(2 Pt 1):359-72.

5. van Kuijk SM, Nijdam ME, Janssen KJ, Sep SJ, Peeters LL, Delahaije DH, et al. A model for preconceptional prediction of recurrent early-onset preeclampsia: derivation and internal validation. Reprod Sci. 2011 Nov;18(11):1154-9.

6. Delahaije DH, van Kuijk SM, Dirksen CD, Sep SJ, Peeters LL, Spaanderman ME, et al. Cost-effectiveness of recurrence risk guided care versus care as usual in women who suffered from early-onset preeclampsia including HELLP syndrome in their previous pregnancy (the PreCare study). BMC pregnancy and childbirth. 2010;10:60.

7. EuroQol--a new facility for the measurement of health-related quality of life. The EuroQol Group. Health Policy. 1990 Dec;16(3):199-208.

8. Dolan P, Roberts J. Modelling valuations for Eq-5d health states: an alternative model using differences in valuations. Medical care. 2002 May;40(5):442-6.

9. Hakkaart-van Roijen L, Tan SS, Bouwmans CAM. Handleiding voor kostenonderzoek. Methoden en standaard kostprijzen voor economische evaluaties in de gezondheidszorg. Amstelveen: College voor Zorgverzekeringen (CVZ); 2010. 
10. (CBS) CBvdS. Centraal Bureau voor de Statistiek: Consumentenprijzen. Available from: http://statline.cbs.nl/StatWeb/publication/?VW=T\&DM=SLNL\&PA=71311NED\&D1=0,2,4,6\&D2=0-

1,61,70,87,108,137,145,172,176,221-222,230,255,I\&D3=(I-34)-I\&HD=081020-1310\&HDR=T\&STB=G1,G2.

11. Oostenbrink JB, Bouwmans CAM, Koopmanschap MA, Rutten FFH. Handleiding voor kostenonderzoek: Methoden en standaard kostprijzen voor economische evaluaties in de gezondheidszorg. . Rotterdam: College voor zorgverzekeringen; 2004.

12. zorgverzekeringen Cv. The Genees- en hulpmiddelen Informatie Project Databank http://www.gipdatabank.nl: College voor zorgverzekeringen; [cited 2011].

13. Schafer JL, Graham JW. Missing data: our view of the state of the art. Psychological methods. 2002 Jun;7(2):147-77.

14. van Hout BA, Al MJ, Gordon GS, Rutten FF. Costs, effects and C/E-ratios alongside a clinical trial. Health economics. 1994 Sep-Oct;3(5):309-19.

15. van Asselt $A D$, van Mastrigt GA, Dirksen CD, Arntz A, Severens JL, Kessels AG. How to deal with cost differences at baseline. PharmacoEconomics. 2009;27(6):519-28.

16. Sep S, Smits L, Prins M, Peeters L. Prediction tests for recurrent hypertensive disease in pregnancy, a systematic review. Hypertension in pregnancy : official journal of the International Society for the Study of Hypertension in Pregnancy. 2010 Jan;29(2):206-30.

17. van Kuijk SM, Delahaije DH, Dirksen CD, Scheepers HC, Spaanderman ME, Ganzevoort W, et al. Multicenter external validation and recalibration of a model for preconceptional prediction of recurrent early-onset preeclampsia. Submitted for publication.

18. van Kuijk SM, Delahaije DH, Dirksen CD, Scheepers HC, Spaanderman ME, Ganzevoort W, et al. Multicenter impact analysis of a model for predicting recurrent early-onset preeclampsia. Results of the PreCare study. Submitted for publication. 



\section{CHAPTER 5}

\section{Patient satisfaction with recurrence risk guided}

care in pregnant women with previous preeclampsia or HELLP syndrome

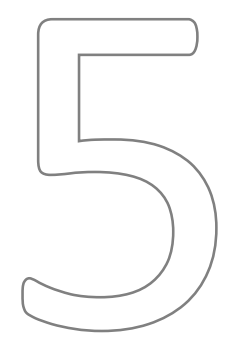

Denise HJ Delahaije, Luc J Smits, Sander MJ van Kuijk, Louis L Peeters, Johannes J Duvekot, Wessel Ganzevoort, Martijn A Oudijk, Mariëlle G van Pampus, Hubertina CJ Scheepers, Marc E Spaanderman, Carmen D Dirksen (a) for the PreCare study group 


\begin{abstract}
Background

It has become evident that patient satisfaction is an important issue to consider when optimising the (cost-)effectiveness of a health care innovations, as patients' opinions will help shape, improve and implement the innovation. This study aimed to investigate the level of satisfaction with recurrence risk guided care in pregnant women who had suffered from preeclampsia or HELLP syndrome in their previous pregnancy. Other objectives were to determine which factors contribute to the level of satisfaction and whether level of satisfaction has an influence on protocol adherence.
\end{abstract}

\title{
Methods
}

This patient satisfaction study is part of the larger PreCare study, investigating the effectiveness and cost-effectiveness of recurrence risk guided care versus care as usual. Patients were treated in six university hospitals and seven large non-university tertiary referral hospitals in the Netherlands between 2008 and 2012. Patient satisfaction was measured by the Patient Satisfaction Questionnaire (PSQ III) at baseline and 10 days postpartum. Based on Crow's holistic model of satisfaction, multivariate regression analyses were performed to investigate which factors (i.e. baseline level of satisfaction, level of care (i.e. High Care or Medium Care), socio-demographic variables, health state at baseline, previous experiences and expectations) contribute to the level of satisfaction 10 days postpartum and which subscales of the PSQ III contribute to protocol adherence.

\section{Results}

Patient satisfaction scores were high on all subscales of the PSQ III. Factors that explained variation in patient satisfaction were level of care, psychological health state at baseline, expectations and baseline patient satisfaction score. Protocol adherence was poor and after controlling for other factors, only associated with the "communication" subscale.

\section{Conclusion}

Although we lack a comparison with care-as-usual, our findings indicate that, overall, patients were satisfied with recurrence risk guided care. Satisfaction scores were moderately well explained by factors contained in Crow's model. Patient satisfaction was, however, a poor predictor of protocol adherence, probably because health care providers' and organisational characteristics are important co-determinants of protocol adherence. 


\section{Background}

Quality of care has gained greater prominence in recent years and there is a growing body of evidence in this field of research [1-3]. Quality of care can objectively be assessed by examining clinical practice in terms of process and outcome indicators and subjectively by obtaining patient experience indicators, like patient satisfaction [2, 4, 5]. Examining quality of care through the patients' eyes can reveal important information, because professional and expert views about the care provided can vary markedly from patient judgements [6, 7]. Monitoring patient satisfaction is important because if the quality of the care provided is evaluated regularly, problem areas can be isolated and ideas for improvement can be generated [8, 9]. Furthermore, as satisfied patients are more positive about their situation, they tend to respond better to treatment and are more compliant and cooperative, improving their health outcomes [1012].

Women with high risk pregnancies have been a particular target group for investigating patient satisfaction. Pregnancy in this patient group can be a stressful and dissatisfying experience which generally results in increased health care consumption [1319]. Furthermore, previous studies have shown that the quality of prenatal care is an important determinant of pregnancy outcome [20,21].

The focus of this paper is on patient satisfaction in a sample of pregnant women with a history of preeclampsia (PE) or HELLP syndrome who participated in a multicentre before-after study investigating the effectiveness and cost-effectiveness of recurrence risk guided care $(R G C)$ versus care-as-usual (CAU), the PreCare study. Details on the design of the study have been reported previously [22]. For RGC, we developed a model for the prediction of recurrent PE/HELLP in the next pregnancy for formerly preeclamptic women. Based on the prediction model, women were stratified into subgroups to be allocated to Medium Care (MC) or High Care ( $\mathrm{HC})$, according to their actual risk profile. In this study, we measured patient satisfaction with RGC. Patient satisfaction with risk-adjusted antenatal care is of interest to practitioners and policy makers because user acceptability will optimise the (cost-)effectiveness of RGC, e.g. through protocol adherence, and will enhance its broader implementation. Furthermore, areas for improvement can be identified.

In this study, we addressed the following research questions:

1. What is the level of satisfaction of patients who receive RGC?

2. Which factors contribute to the level of satisfaction?

3. Does level of satisfaction have an influence on protocol adherence? 


\section{Methods}

\section{Recruitment, design and sample}

This patient satisfaction study is part of the larger before-after PreCare study. The PreCare study is embedded in the Dutch Obstetric Consortium, a collaboration of hospitals in the Netherlands [23].

Between 2008 and 2012, pregnant women were recruited through six university hospitals and seven large non-university tertiary referral hospitals in the Netherlands. Participants were eligible if they were 18 years and older, had PE and/or HELLP syndrome in their previous pregnancy and visited the outpatient clinic until 20 weeks of pregnancy. Exclusion criteria were severe co-morbidity (including diabetes mellitus, systemic lupus erythematosus, renal disease, cardiac disease or anti-phospholipids syndrome) and giving birth after 37 weeks gestational age in the previous pregnancy. All eligible patients received detailed information about the study.

The study protocol was approved by the Medical Ethical Review Board of the Maastricht University Medical Centre (NL). All participating centres signed a local feasibility declaration, according to the Dutch law and regulations, prior to inclusion of the first patient.

\section{A holistic model of patient satisfaction}

Despite the increased focus on patient satisfaction in recent years, there is still no agreement on its definition. Hulka et al., 1970 defined satisfaction as the patient's attitudes toward physicians and medical care [24]. Another definition of patient satisfaction is the attitudinal response to value judgments that patients make about their clinical encounter [25]. Linder-Pelz defined patient satisfaction as evaluations of distinct dimensions of the health care [26]. In this paper, we use the latter definition of patient satisfaction.

Several approaches have been used to try to identify the factors contributing to satisfaction, e.g. approaches based on expectation, approaches based on health services attributes and economic approaches [9]. Holistic approaches attempt to incorporate all influences on satisfaction and thereby to provide a comprehensive framework for exploring interactions between variables that affect patients' evaluations [9]. Crow et al., 2002 [9] presented a holistic model, adapted from Strasser and Davis (1991) [27] and Strasser et al. (1993) [28].

As a conceptual framework for answering our research questions, we made use of Crow's holistic model of patient satisfaction [9]. According to this model, patients experience individual stimuli such as actions, attitudes and appearances of human re- 
sources, the physical environment and organisational aspects of care (Box A). Then, perceptions are created through cognitive and affective processes (Box $B$ ), which are specific to individual patients, reflecting their particular individual characteristics (Box C). (Dis)satisfaction is viewed as a reflection of the difference between what is expected and what is perceived to have been delivered. If the expectations exceed the experiences, the patient will be dissatisfied; if the experiences exceed the expectations, the patient will be satisfied [9].

Satisfaction is an individual attitudinal response to the value judgments formed before (Box D). Satisfaction or dissatisfaction has consequences for both the patient and the provider (Box E). On patient level, satisfaction is linked with adherence to medical advice, self-care, and hence with health outcomes. Furthermore, satisfied patients will use the service again and recommend it to others. The model described above is shown in Figure 1.

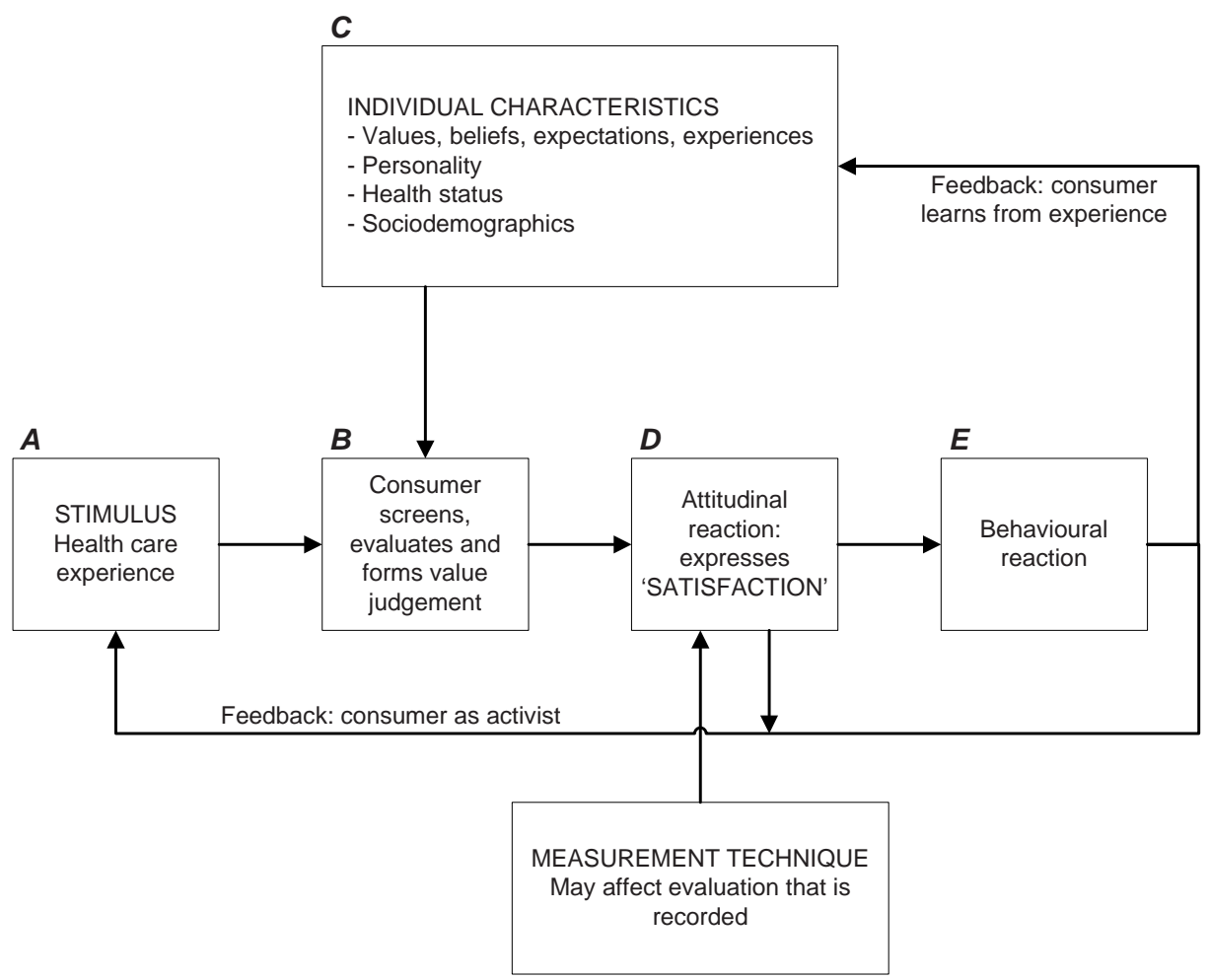

Figure 1: Holistic model of satisfaction with health care, adapted from Crow et al., 2002 [9] 


\section{Procedures and intervention (Box A)}

At the first visit at the outpatient clinic, after written informed consent was obtained, the risk of severe recurrent PE/HELLP was calculated. For risk calculation, an easily applicable computer programme was developed and made available for the participating gynaecologists and research nurses. On the basis of the predicted risk, the patient was allocated to either the Medium Care or the High Care protocol.

The Medium Care protocol consisted of 9-11 outpatient clinic visits at $(8,12), 16$, $20,24,28,32,36,38,39$ and 40 weeks gestational age. The High Care protocol consisted of 14-16 outpatient clinic visits at $(8,12), 16,18,20,22,24,26,28,30,32,34$, $36,38,39$ and 40 weeks gestational age. In the High Care group, the patient additionally underwent a series of diagnostic tests, which consisted of measurements in urine (e.g. microalbuminuria, creatinine, and protein), measurements in blood (e.g. haemoglobin, platelet count, and glucose), and assessment of the Doppler flow velocity profile in both uterine arteries at 20 weeks. Ambulatory blood pressure monitoring was suggested but not obligatory. Activities other than, or additional to those described in the Medium Care and High Care protocols (such as intercurrent admissions and additional outpatient visits) were also recorded.

\section{Measurement of patient satisfaction (Box D)}

In order to assess patient satisfaction, the Dutch version of the validated Ware's Patient Satisfaction Questionnaire III (PSQ III) was used, which was filled out at baseline and 10 days postpartum. The PSQ III measures the multiple concept of patient satisfaction and incorporates "general satisfaction", "technical quality", "interpersonal aspects", "communication", "financial aspects", "time spent with doctor" and "access/availability/convenience" [29]. In the Dutch version, the financial items are omitted because these are not appropriate for the Dutch socialised system (i.e. questions 4 , $10,14,19,24,27,32$ and 44$)$. As a result, the questionnaire consists of 43 favourably and unfavourably worded statements, in which respondents are asked to indicate their agreements with the statements with respect to the care they received. The questionnaire was scored with five response categories ranging from 1 (strongly agree) to 5 (strongly disagree). Answers to favourably worded items were recoded so that higher scores indicate greater satisfaction. Sum scores were calculated for the six subscales and subsequently transformed into a 100-point scale so that subscale scores are directly comparable to one another despite different numbers of items on each scale.

As an additional aspect for rating satisfaction with the given care according to the monitoring protocols, a specific questionnaire was developed inspired by the Women's questionnaire in the antenatal care trial performed by the World Health Organization [30]. At baseline, this PreCare satisfaction questionnaire contained seven statements 
concerning the expectations regarding $\mathrm{RGC}$ regarding the number of outpatient visits ( 2 statements), the time between visits ( 3 statements) and RGC (3 statements). Patients were asked to indicate on a 5-point Likert scale whether they fully agreed (1) or fully disagreed (5) with each of the statements. Finally patients were asked to indicate the overall satisfaction regarding antenatal care on a scale from 1 (very dissatisfied) to 10 (very satisfied). Answers to favourably worded items were recoded so that higher scores indicate more positive expectations. Subsequently, a sum score was calculated. The sum score ranges from 7 to 35 with higher scores reflecting more positive expectations. The questionnaires were sent to the patients' homes and requested to be returned by postal mail.

\section{Individual characteristics (Box C)}

Selection of patient characteristics that might affect patient satisfaction was based upon Crow's model and availability of data from the PreCare study. The patient variables that were considered in this patient satisfaction study can be grouped into four areas: socio-demographic factors, health state at baseline, previous experiences and expectations. The first category, socio-demographics, includes information about age (in years), country of birth (Netherlands or other) and educational level (low: primary, junior secondary, senior secondary; and high: higher professional or university education). This information was obtained by a questionnaire sent at approximately 29 weeks of pregnancy. Additionally, information about health state at baseline (i.e. health-related quality of life and psychopathology) was collected. At inclusion, all patients completed a questionnaire on health-related quality of life and were evaluated for DSM-IV depressive, anxiety and posttraumatic stress disorder (PTSD) symptoms. Health-related quality of life was measured with the EuroQol (EQ-5D) [31]. The descriptive system of the EQ-5D consists of five dimensions (mobility, self-care, daily activities, pain/discomfort, depression/anxiety). Each dimension has three response options: no problems (1), some problems (2), severe problems (3), leading to 243 possible health states. For each health state, a utility score can be computed, based on preferences from a general Dutch population, i.e. the Dutch tariff. Depressive, anxiety and posttraumatic stress disorder (PTSD) symptoms were measured with the Beck Depression Inventory (BDI) [32], the State Trait Anxiety Inventory (STAI) [33] and the PTSD Symptom Scale [34] respectively. Scores on the depression scale range from 0 to 63. Higher scores correlate with greater depression: 0-9 minimal depression, 10-18 mild depression, 19-29 moderate depression, 30-63 severe depression. Scores on the anxiety scale range from 20 to 80 . Higher scores correlate with greater anxiety. Scores on the PTSD scale range from 0 to 51 . A total score higher than 13 indicates a likely presence of PTSD. Previous experiences (characteristics of the previous pregnancy: 
gestational age at delivery and small-for-gestational age) were collected from patient files and recorded in online Case Report Forms (CRFs), which were specifically designed for the PreCare study. The last group of variables, called "expectations", was measured by the PreCare satisfaction questionnaire, and we corrected also for PSQ III baseline scores. Values, beliefs and personality were not measured.

\section{Protocol adherence (Box E)}

Data on actual care received, i.e. number of hospital visits and diagnostic measurements, were recorded in online CRFs. First, we calculated patient-level protocol adherence scores by summing the actual number of outpatient visits. The required number of outpatient visits according to the $\mathrm{MC}$ or $\mathrm{HC}$ protocol was adjusted for gestational age at delivery, since women with preterm deliveries are likely to have less outpatient visits than women with term deliveries. Then, we compared the actually received number of visits per patient with the adjusted number of visits described in the MC or $\mathrm{HC}$ protocol (depending on the patient's risk). Protocol adherence was a continuous variable. The adherence score is positive for the number of times the patient had had an outpatient visit exceeding the number of visits described in the protocol; the adherence score is zero if the number of times the patient had a visit is lower than or equal to the number of visits described in the protocol.

\section{Statistical analysis}

Descriptive statistics, including frequencies, percentages, means and standard deviations (SDs), were used to describe the population at baseline. Women who did not return any patient satisfaction questionnaire were compared with women who returned at least one patient satisfaction questionnaire with respect to important variables (maternal characteristics and characteristics of the previous pregnancy) using a Chi-square test for categorical variables and a t-test for continuous variables. Missing values within the survey's subscales were replaced using single imputation.

In order to investigate which factors contribute to the level of satisfaction at 10 days postpartum, candidate variables (i.e. HC/MC, sociodemographic variables, health state at baseline, previous experiences and expectations) were identified by including them in a multivariate regression model using a backward selection procedure. This automated backward stepwise procedure implied dropping statistically non-significant independent variables from the model using a significance level of $\alpha=0.10$. The variables "education" and "country of birth" had missing values in 21 patients; both variables were statistically non-significant among the 63 patients without missing values. The final model was therefore produced among patients with and without missing 
values on these variables $(n=84)$ by forcing all variables into the model that were statistically significant among the smaller sample. Regression coefficients in the smaller and larger sample were similar, indicating the robustness of the results.

In order to investigate which subscales of the PSQ III contribute to protocol adherence, all subscales (i.e. "general satisfaction", "technical quality", "interpersonal aspects", "communication", "time spent with doctor" and "access/availability/convenience") were introduced into a multivariate regression model and maintained or dropped using backward selection $(\alpha=0.10)$. We controlled for confounding variables (see previous regression analysis: $\mathrm{HC} / \mathrm{MC}$, sociodemographic variables, health state at baseline, previous experiences and expectations). Again, regression coefficients in the smaller and larger sample were similar. Collinearity between the variables in the model was assessed by examining the tolerance and variance inflation factor for each independent variable. Normality of the residuals was checked by use of a normal probability plot of the residuals. Significance tests of all other analyses were two-tailed and $\alpha$ was set at 0.05. All analyses were performed using SPSS 20.0.

\section{Ethical considerations}

This study has been approved by the ethical committee of the University Hospital Maastricht (Ref.no. MEC 07-2-078). A total of six academic and seven non-academic hospitals participate in the study; all hospitals completed the obligatory feasibility assessment procedure successfully. Informed consent was obtained from all patients prior to enrolment into the study.

\section{Results}

\section{Patients}

In total, 159 RGC patients participated in the larger PreCare study. The first RGC patient was included in June 2009 and the last one in May 2012. We introduced the patient satisfaction questionnaires some time after commencement of the study, the first patient satisfaction questionnaire was filled out in January 2010. Of the total sample of 159, 103 patients were offered the satisfaction questionnaires. Eighty-four women $(81.6 \%)$ returned at least one satisfaction questionnaire and were included in the present study. Baseline characteristics of the women are presented in Table 1 . There were no relevant differences between respondents and non-respondents with respect to maternal characteristics and characteristics of the previous pregnancy, except for country of birth. 
Table 1: Characteristics of women treated according to RGC

\begin{tabular}{|c|c|c|}
\hline & $\begin{array}{l}\text { Respondents } \\
(n=84)\end{array}$ & $\begin{array}{l}\text { Non-respondents } \\
(n=19)\end{array}$ \\
\hline \multicolumn{3}{|l|}{ Maternal characteristics } \\
\hline Maternal age at conception (yrs) & $31.4(4.5)$ & $31.7(5.2)$ \\
\hline Body mass index $\left(\mathrm{kg} / \mathrm{m}^{2}\right)$ & $25.7(6.2)$ & $25.4(5.3)$ \\
\hline Gravidity & $2.5(0.8)$ & $2.5(0.6)$ \\
\hline Parity & $1.1(0.4)$ & $1.2(0.5)$ \\
\hline Hypertension, n (\%) & $29(34.5)$ & $5(26.3)$ \\
\hline \multicolumn{3}{|l|}{ Country of birth } \\
\hline Netherlands, n (\%) & $58(69.1)$ & $9(47.4)$ \\
\hline Other, n (\%) & $5(6.0)$ & $4(21.1)$ \\
\hline Missing, $\mathrm{n}(\%)$ & $21(25.0)$ & $6(31.6)$ \\
\hline \multicolumn{3}{|l|}{ Educational level } \\
\hline Low, n (\%) primary school, junior/senior secondary & $38(45.2)$ & $7(36.8)$ \\
\hline High, n (\%) higher professional, university & $25(29.8)$ & $6(31.6)$ \\
\hline Missing, $\mathrm{n}(\%)$ & $21(25.0)$ & $6(31.6)$ \\
\hline Utility score baseline & $0.8(0.3)$ & $0.8(0.3)$ \\
\hline PTSD score baseline & $9.1(6.7)$ & $7.3(6.9)$ \\
\hline Anxiety score baseline & $46.8(11.4)$ & $46.2(10.6)$ \\
\hline Depression score baseline & $10.3(5.9)$ & $9.2(7.6)$ \\
\hline \multicolumn{3}{|l|}{ Characteristics of previous pregnancy } \\
\hline Gestational age (weeks) & $31.2(3.7)$ & $31.8(4.1)$ \\
\hline PE, n (\%) & $73(86.9)$ & $17(89.5)$ \\
\hline HELLP syndrome, n (\%) & $50(59.5)$ & $9(47.4)$ \\
\hline Eclampsia, n (\%) & $3(3.6)$ & $1(5.3)$ \\
\hline IUFD, n (\%) & $8(9.5)$ & $3(15.8)$ \\
\hline Birth weight (gr) & $1398.7(721.9)$ & $1574.7(840.8)$ \\
\hline IUGR, n (\%) & $30(35.7)$ & $9(47.4)$ \\
\hline
\end{tabular}

Data are given as mean \pm SD or as percentages

* Significant differences

\section{The level of satisfaction}

Table 2 shows the satisfaction scores measured 10 days postpartum, on a scale ranging from 0 to 100. Ten days postpartum all but one patient satisfaction score were in the top- $25 \%$ of the PSQ III subscales. Lowest level of satisfaction was observed for the "time spent with doctor" subscale (score 74.22). This subscale includes items about whether doctors spend enough time or hurry too much when treating patients. The 
highest level of satisfaction was reached for the subscale "interpersonal aspects" (score 87.32).

Table 2: Average satisfaction scores

\begin{tabular}{ll}
\hline & $\begin{array}{l}\text { All patients }(\mathrm{n}=84) \\
\text { Mean }(S D ; \text { range })\end{array}$ \\
\hline General satisfaction & $76.8(14.9 ; 29.2-100.0)$ \\
Technical quality & $77.9(12.2 ; 40.0-100.0)$ \\
Interpersonal aspects & $87.3(10.3 ; 50.0-100.0)$ \\
Communication & $85.9(11.0 ; 50.0-100.0)$ \\
Time spent with doctor & $74.2(20.7 ; 12.5-100.0)$ \\
Access/availability/convenience & $75.2(10.9 ; 33.3-97.9)$ \\
\hline
\end{tabular}

\section{Factors contributing to the level of satisfaction}

The results of the multiple regression analyses for those variables that were most predictive of patient satisfaction 10 days postpartum are shown in Table 3.

Patients with higher depression scores at baseline were more satisfied 10 days postpartum with "technical competence", "interpersonal aspects", "time spent with doctor" and "access". Anxiety at baseline was negatively associated with the satisfaction subscales "technical competence", "interpersonal aspects", "communication" and "access". PTSD was associated with three satisfaction subscales "time spent with doctor", "interpersonal aspects" and "access", although in an inconsistent manner.

Remarkably, level of care $(\mathrm{HC} / \mathrm{MC})$ was significantly related to the subscale "time spent with doctor" in that patients in the HC group were considerably more satisfied (11 points) than patients in the MC group. Expectations at baseline were positively associated with "general satisfaction" and satisfaction with "technical competence", whereas PSQ III baseline scores were positively associated with "interpersonal aspects" and "time spent". Overall, depression and PTSD at baseline can be said to have the most potent relationship to level of satisfaction, because of the largest standardised beta and the greatest significance (smallest $p$-value). 
Table 3: Multivariate regression analysis of the association of various factors with patient satisfaction scores

\begin{tabular}{|c|c|c|c|c|}
\hline Dependent variable & Explanatory variables & $\begin{array}{l}\text { Unstandardised } \\
\text { B (SE) }\end{array}$ & $\begin{array}{l}\text { Standardised } \\
\beta\end{array}$ & p-value \\
\hline Gen. satisfaction & $\begin{array}{l}\text { Expectations baseline (PreCare satisfaction } \\
\text { questionnaire sum score) }\end{array}$ & $0.637(0.313)$ & 0.219 & 0.045 \\
\hline \multirow[t]{3}{*}{ Techn. comp. } & Depression score baseline & $0.320(0.274)$ & 0.155 & 0.246 \\
\hline & Anxiety score baseline & $-0.262(0.142)$ & -0.244 & 0.069 \\
\hline & $\begin{array}{l}\text { Expectations baseline (PreCare satisfaction } \\
\text { questionnaire sum score) }\end{array}$ & $0.498(0.260)$ & 0.208 & 0.059 \\
\hline \multirow[t]{4}{*}{ Interpers.asp. } & Depression score baseline & $0.969(0.279)$ & 0.556 & 0.001 \\
\hline & Anxiety score baseline & $-0.166(0.117)$ & -0.183 & 0.160 \\
\hline & PTSD score baseline & $-0.614(0.230)$ & -0.401 & 0.009 \\
\hline & Interpersonal aspects baseline score & $0.217(0.075)$ & 0.321 & 0.005 \\
\hline Communication & Anxiety score baseline & $-0.224(0.104)$ & -0.231 & 0.034 \\
\hline \multirow[t]{4}{*}{ Time spent } & $\mathrm{HC} / \mathrm{MC}(0=\mathrm{HC} ; 1=\mathrm{MC})$ & $-11.004(4.693)$ & -0.247 & 0.022 \\
\hline & Depression score baseline & $1.441(0.551)$ & 0.421 & 0.011 \\
\hline & PTSD score baseline & $-0.178(0.461)$ & -0.383 & 0.012 \\
\hline & Time spent with doctor baseline score & $0.247(0.105)$ & 0.266 & 0.021 \\
\hline \multirow[t]{3}{*}{ Access } & Depression score baseline & $0.432(0.231)$ & 0.235 & 0.066 \\
\hline & Anxiety score baseline & $-0.352(0.122)$ & -0.369 & 0.005 \\
\hline & PTSD score baseline & $0.201(0.087)$ & 0.251 & 0.023 \\
\hline
\end{tabular}

\section{Protocol adherence}

Table 4 shows the protocol adherence. Seventy out of 84 patients (83.3\%) had more outpatient visits than prescribed in the $\mathrm{HC}$ or MC protocol (adjusted for gestational age at delivery). In most patients, the number of outpatient visits was exceeded with 1-5. The model of Crow, presented earlier, states that patient satisfaction is linked with, among others, adherence. After adjustment for confounding variables, only satisfaction with "communication" appeared to be a predictor of adherence score in a backward multivariate regression analysis assessing all patient satisfaction subscales $(B=0.097 ; p=0.075)$. Being more satisfied with "communication" was significantly related to more outpatient visits. However, only $16.4 \%$ of the variance in patient satisfaction $\left(\mathrm{R}^{2}\right)$ was explained by "communication". 
Table 4: Frequency of visits (percentage of patients)

Non-adherent patients $(n=70)$

Number of visits exceeding the number described in the protocol, $n$ (\%)

\begin{tabular}{ll}
\hline $1-5$ visits & $37(44.0)$ \\
$6-10$ visits & $18(21.4)$ \\
$>10$ visits & $15(17.9)$
\end{tabular}

\section{Discussion}

Patient satisfaction is an important issue to consider when aiming to optimise the (cost-)effectiveness of a new treatment option, as patients' opinions will help shape, improve and implement the treatment option [9]. Insight into patient satisfaction was therefore an important aspect of the PreCare study. Patient satisfaction scores seemed high in all subscales of the PSQ III as scores on most subscales were in the top- $25 \%$. High satisfaction scores do not imply that there is no room for improvement. In this study we observed differences between the satisfaction subscales. In general, patients were most satisfied with "interpersonal aspects" and "communication", and least satisfied with "time spent" and "access". Several studies have used (parts of) the PSQ III. The reported satisfaction scores in other studies may be used as some kind of a benchmark for this study, although it should be noted that they apply to different populations and care settings. The study of Hagedoorn et al. in 2,594 Dutch cancer patients reported mean satisfaction scores on "technical competence" of 78.2 and 80.3 on "access" [35]. Another Dutch study in 299 cancer patients reported mean scores on "general satisfaction" of 76.4 (telephone follow-up treatment arm) and 75.3 (hospital follow-up treatment arm), on "technical competence" of 75.8 and 73.7, on "interpersonal aspects" of 80.5 and 78.7 and on "access" of 76.4 and 73.3 [36]. Thomas et al., 2009 found in 653 cancer patients in Canada mean "general satisfaction" scores ranging from 55.5 to 69.3 , depending on ethnicity and other demographic characteristics [37]. The study of Marshall et al., conducted in 417 patients with significant symptoms of depression and 535 with chronic physical health conditions in the United States, found mean scores on "technical competence" of 64.05 and 70.83 , mean scores on "interpersonal aspects" of 68.90 and 76.12, mean scores on "communication" of 66.91 and 74.39, mean scores on "time spent" of 58.39 and 66.80 and mean scores on "access" of 65.95 and 73.01 [38]. These figures indicate that, in general, our patient group was quite satisfied in comparison to those of other studies. Studies conducted in the United States and Sweden showed a higher patient satisfaction with gynaecologyobstetrics compared with other specialties $[39,40]$. 
Besides examining the level of satisfaction, more importantly, this study provides insight in factors relating to patient satisfaction. The most consistent finding in the literature regarding these factors has been related to age. Older patients tend to be more satisfied than younger patients [40-43]. In our study, however, age did not contribute to patient satisfaction. This finding is possibly due to the small variance in age in our sample of pregnant women (between $20-40$ years). Furthermore, being a member of a minority group is associated with lower rates of satisfaction [44]. Less educated individuals and those with a lower socioeconomic status tend to be less satisfied [45]. In this study, "country of birth" and "education" were not included in the final regression model because of the large number of missing values on these variables. Current evidence also shows that patients with a lower health state report more problems with the health care system [46]. Some studies have shown that poorer satisfaction is associated with depression, as is having a psychiatric diagnosis such as posttraumatic stress disorder [47-49]. Although some studies have reported associations between these individual characteristics and patient satisfaction scores, the evidence has been generally inconclusive [9]. Patients' expectations are believed to be another major determinant of satisfaction with health care $[50,51]$. It is suggested that patients with lower expectations and patients with a greater number of met expectations tend to be more satisfied [8]. In this study, it was found that patients with more positive expectations are more satisfied, which indicates that their expectations had been met. It is important to highlight that various factors previously reported to influence patient satisfaction did not contribute to the variation in patient satisfaction in this study. Only patients' expectations, baseline psychological health state and being assigned to HC or $\mathrm{MC}$ had a significant impact on aspects of satisfaction. Remarkably, whether women had been assigned to either $\mathrm{HC}$ or MC was significantly related to satisfaction regarding "time spent with doctor". Patients in the HC group were more satisfied with "time spent with doctor" than patients in the MC group, perhaps because in the MC protocol as compared to $\mathrm{HC}$, the number of outpatient visits and diagnostic procedures was restricted. As a result, women in the MC group may have perceived a loss in terms of number of outpatient visits and time spent with doctor. In economics, this phenomenon is referred to as loss aversion [52].

Adherence to the $\mathrm{HC}$ and $\mathrm{MC}$ protocols was suboptimal, despite considerable efforts to promote and support them. Protocol adherence (i.e. the excess number of outpatient visits) was only significantly explained by the "communication" subscale of the PSQ III questionnaire, although its parameter value indicates that the actual contribution of "communication" to protocol adherence is quite limited. The course of antenatal care takes place through close interaction and shared decision-making between the gynaecologist and the patient. Many factors influence the adherence to a guideline in daily practice. We investigated several patient related factors (i.e. recur- 
rence $\mathrm{PE} / \mathrm{HELLP}$, depression, anxiety, posttraumatic stress, health-related utility at baseline, the level of satisfaction) and these were not associated with the (excess) number of outpatient visits. However, apart from patient-related factors, barriers to guideline adherence may be related to the individual health care professional, the social context and the economic, administrative and organisational context [53]. For example, the implementation of a new guideline might be unsuccessful if there is a false idea among medical specialists that patients need constant reassurance and a long-standing belief that considering costs is unethical, if colleagues do not apply the new guideline, if medical specialists question the scientific arguments of the guidelines or do not have faith in a successful process. Furthermore, some participating gynaecologists already used to work with prediction models in their clinic and the confidence of these doctors in our prediction model might have been influenced by previous experiences with other prediction models. Crow's holistic model is developed from the patient's perspective and does not include health care provider's and organisational characteristics, so it will only partly explain adherence. In our study, we did not measure these additional factors. An adequate analysis of these potential barriers is an important step in improving guideline adherence and, subsequently, quality of care.

\section{Strengths and limitations}

Our study findings are strengthened by the study design, a multicentre study, which contributes to the generalisability of the results. Valid and reliable instruments for assessing patient satisfaction and clinical outcomes were used, data were collected prospectively with baseline measurements of health state, expectancies and previous experiences. Furthermore, factors included in the regression analyses were based on a published holistic model of patient satisfaction.

A limitation of the study is that we could not compare satisfaction between the CAU and the RGC group because we did not measure satisfaction in the CAU group. The reason for this is that the larger part of the CAU data were obtained from a historical cohort of patients. If RGC leads to a reduction in costs compared to CAU, we cannot conclude that RGC patients are also more satisfied than CAU patients. However, in this non-comparative study, RGC patients appeared overall satisfied with the care they received and this is an important indicator of patient acceptability when implementing RGC in clinical practice. Second, the finding that only few factors were associated with the patient satisfaction subscales and protocol adherence may partly be caused by the relatively small sample size. A larger sample size could possibly lead to a statistically significant relationship by smaller confidence intervals, leading to potential rejection of the null hypothesis (i.e. that there is no relationship). Finally, although extensive clinical and sociodemographic data were collected, there are other patient-related factors 
(such as values, beliefs and personality) which were not assessed in this study but that could affect satisfaction.

\section{Conclusions}

This study shows that patient satisfaction scores were high in terms of "general satisfaction", "technical quality", "interpersonal aspects", "communication", "time spent with doctor" and "access, convenience and availability". Overall, HC/MC, psychological health state at baseline, expectations and baseline patient satisfaction scores had a significant impact on patient satisfaction scores 10 days postpartum. Only the subscale "communication" had a significant impact on protocol adherence.

\section{References}

1. Cleary PD, Edgman-Levitan S: Health care quality. Incorporating consumer perspectives. JAMA : the journal of the American Medical Association 1997, 278(19):1608-1612.

2. Brook RH, McGlynn EA, Cleary PD: Quality of health care. Part 2: measuring quality of care. The New England journal of medicine 1996, 335(13):966-970.

3. Campbell SM, Roland MO, Buetow SA: Defining quality of care. Soc Sci Med 2000, 51(11):1611-1625.

4. Vuori $\mathrm{H}$ : Patient satisfaction--does it matter? Quality assurance in health care : the official journal of the International Society for Quality Assurance in Health Care / ISQA 1991, 3(3):183-189.

5. Vuori H: Patient satisfaction--an attribute or indicator of the quality of care? QRB Quality review bulletin 1987, 13(3):106-108.

6. Merkel WT: Physician perception of patient satisfaction. Do doctors know which patients are satisfied? Medical care 1984, 22(5):453-459.

7. Street RL, Haidet P: How well do doctors know their patients? Factors affecting physician understanding of patients' health beliefs. J Gen Intern Med 2011, 26(1):21-27.

8. Sitzia J, Wood N: Patient satisfaction: a review of issues and concepts. Soc Sci Med 1997, 45(12):18291843.

9. Crow R, Gage H, Hampson S, Hart J, Kimber A, Storey L, Thomas H: The measurement of satisfaction with healthcare: implications for practice from a systematic review of the literature. Health Technol Assess 2002, 6(32):1-244.

10. Kizer KW: Patient centred care: essential but probably not sufficient. Quality \& safety in health care 2002, 11(2):117-118.

11. Worthington K: Customer satisfaction in the emergency department. Emergency medicine clinics of North America 2004, 22(1):87-102.

12. Jackson JL, Chamberlin J, Kroenke K: Predictors of patient satisfaction. Soc Sci Med 2001, 52(4):609-620.

13. Dunlop L, Umstad M, McGrath G, Reidy K, Brennecke S: Cost-effectiveness and patient satisfaction with pregnancy day care for hypertensive disorders of pregnancy. The Australian \& New Zealand journal of obstetrics \& gynaecology 2003, 43(3):207-212.

14. Leeners B, Rath W, Kuse S, Neises M, Neumaier-Wagner P: Satisfaction with medical information in women with hypertensive disorders in pregnancy. Journal of psychosomatic research 2006, 60(1):39-44. 
15. McDuffie RS, Jr., Beck A, Bischoff K, Cross J, Orleans M: Effect of frequency of prenatal care visits on perinatal outcome among low-risk women. A randomized controlled trial. JAMA : the journal of the American Medical Association 1996, 275(11):847-851.

16. El-Kerdawy $\mathrm{H}$, Farouk A: Labor analgesia in preeclampsia: remifentanil patient controlled intravenous analgesia versus epidural analgesia. Middle East journal of anesthesiology 2010, 20(4):539-545.

17. Kuklina EV, Ayala C, Callaghan WM: Hypertensive disorders and severe obstetric morbidity in the United States. Obstetrics and gynecology 2009, 113(6):1299-1306.

18. Liu A, Wen SW, Bottomley J, Walker MC, Smith G: Utilization of health care services of pregnant women complicated by preeclampsia in Ontario. Hypertension in pregnancy : official journal of the International Society for the Study of Hypertension in Pregnancy 2009, 28(1):76-84.

19. Galtier-Dereure F, Boegner C, Bringer J: Obesity and pregnancy: complications and cost. The American journal of clinical nutrition 2000, 71(5 Suppl):1242S-1248S.

20. Blondel B, Dutilh P, Delour M, Uzan S: Poor antenatal care and pregnancy outcome. European journal of obstetrics, gynecology, and reproductive biology 1993, 50(3):191-196.

21. Cohen JR: Patient satisfaction with the prenatal care provider and the risk of cesarean delivery. American journal of obstetrics and gynecology 2005, 192(6):2029-2032; discussion 2032-2024.

22. Delahaije DH, van Kuijk SM, Dirksen CD, Sep SJ, Peeters LL, Spaanderman ME, Bruinse HW, de WitZuurendonk LD, van der Post JA, Duvekot JJ et al: Cost-effectiveness of recurrence risk guided care versus care as usual in women who suffered from early-onset preeclampsia including HELLP syndrome in their previous pregnancy (the PreCare study). BMC pregnancy and childbirth 2010, 10:60.

23. The Consortium Obstetric Research Network [http://studies-obsgyn.nl]

24. Hulka BS, Zyzanski SJ, Cassel JC, Thompson SJ: Scale for the measurement of attitudes toward physicians and primary medical care. Medical care 1970, 8(5):429-436.

25. Kane RL, Maciejewski M, Finch M: The relationship of patient satisfaction with care and clinical outcomes. Medical care 1997, 35(7):714-730.

26. Linder-Pelz SU: Toward a theory of patient satisfaction. Soc Sci Med 1982, 16(5):577-582.

27. Strasser S, Davis R: Measuring patient satisfaction. Ann Arbor, MI: Health Administration Press; 1991.

28. Strasser S, Aharony L, Greenberger D: The patient satisfaction process: moving toward a comprehensive model. Medical care review 1993, 50(2):219-248.

29. Ware JE, Jr., Snyder MK, Wright WR, Davies AR: Defining and measuring patient satisfaction with medical care. Evaluation and program planning 1983, 6(3-4):247-263.

30. Langer A, Villar J, Romero M, Nigenda G, Piaggio G, Kuchaisit C, Rojas G, Al-Osimi M, J.M. MB, Farnot U et al: Are women and care providers satisfied with antenatal care? Views on a standard and a simplified, evidence-based model of care in four developing countries. BMC women's health 2002, 2(7).

31. Anonymus: EuroQoL-a new facility for the measurement of health-related quality of life. The EuroQol Group. Health Policy 1990, 16:199-208.

32. Beck AT, Ward CH, Mendelson M, Mock J, Erbaugh J: An inventory for measuring depression. Archives of general psychiatry 1961, 4:561-571.

33. Spielberger CD, Gorsuch RL, Lushene RE: STAI Manual for the State-trait Anxiety Inventory. Palo Alto, CA: Consulting Psychologists Press; 1970.

34. Foa EB, Riggs DS, Rothbaum B: Reliability and validity of a brief instrument for assessing post traumatic stress disorder. . JTS 1993, 6:459-473.

35. Hagedoorn M, Uijl SG, Van Sonderen E, Ranchor AV, Grol BM, Otter R, Krol B, Van den Heuvel W, Sanderman R: Structure and reliability of Ware's Patient Satisfaction Questionnaire III: patients' satisfaction with oncological care in the Netherlands. Medical care 2003, 41(2):254-263.

36. Kimman ML, Bloebaum MM, Dirksen CD, Houben RM, Lambin P, Boersma LJ: Patient satisfaction with nurse-led telephone follow-up after curative treatment for breast cancer. BMC cancer 2010, 10:174.

37. Thomas BC, Groff SL, Tsang K, Carlson LE: Patient ethnicity: a key predictor of cancer care satisfaction. Ethnicity \& health 2009, 14(4):351-358. 
38. Marshall G, Hays R, Mazel R: Health status and satisfaction with health care: results from the medical outcomes study. Journal of consulting and clinical psychology 1996, 64:380-390.

39. Patel I, Chang J, Srivastava J, Feldman S, Levender M, Balkrishnan R: Patient satisfaction with obstetricians and gynecologists compared with other specialties: analysis of US self-reported survey data. $P a-$ tient related outcome measures 2011, 2:21-26.

40. Rahmqvist $M$ : Patient satisfaction in relation to age, health status and other background factors: a model for comparisons of care units. Int J Qual Health Care 2001, 13(5):385-390.

41. Sixma HJ, Spreeuwenberg PM, van der Pasch MA: Patient satisfaction with the general practitioner: a two-level analysis. Medical care 1998, 36(2):212-229.

42. Jaipaul CK, Rosenthal GE: Are older patients more satisfied with hospital care than younger patients? J Gen Intern Med 2003, 18(1):23-30.

43. Peck BM: Age-related differences in doctor-patient interaction and patient satisfaction. Current gerontology and geriatrics research 2011, 2011:137492.

44. Haviland MG, Morales LS, Dial TH, Pincus HA: Race/ethnicity, socioeconomic status, and satisfaction with health care. American journal of medical quality : the official journal of the American College of Medical Quality 2005, 20(4):195-203.

45. Hall JA, Dornan MC: Patient sociodemographic characteristics as predictors of satisfaction with medical care: a meta-analysis. Soc Sci Med 1990, 30(7):811-818.

46. Covinsky KE, Rosenthal GE, Chren MM, Justice AC, Fortinsky RH, Palmer RM, Landefeld CS: The relation between health status changes and patient satisfaction in older hospitalized medical patients. J Gen Intern Med 1998, 13(4):223-229.

47. Frostholm L, Fink P, Oernboel E, Christensen KS, Toft T, Olesen F, Weinman J: The uncertain consultation and patient satisfaction: the impact of patients' illness perceptions and a randomized controlled trial on the training of physicians' communication skills. Psychosomatic medicine 2005, 67(6):897-905.

48. Desai RA, Stefanovics EA, Rosenheck RA: The role of psychiatric diagnosis in satisfaction with primary care: data from the department of veterans affairs. Medical care 2005, 43(12):1208-1216.

49. Jackson JL, Chamberlin J, Kroenke K: Predictors of patient satisfaction. Soc Sci Med 2001, 52(4):609-620.

50. Thompson A, Sunol R: Expectations as determinants of patient satisfaction: concepts, theory and evidence. Int J Qual Health Care 1995, 7:127-141.

51. Kravitz RL: Patients' expectations for medical care: an expanded formulation based on review of the literature. Medical care research and review : MCRR 1996, 53(1):3-27.

52. Kahneman D, Knetsch J, Thaler R: Anomalies: The endowment effect, loss aversion, and status quo bias. Journal of Economic Perspectives 1991, 5(1):193-206.

53. Grol R, Wensing M, Eccles M: Improving patient care: the implementation of change in clinical practice. Edinburgh: Elsevier Butterworth Heinemann; 2005. 


\section{CHAPTER 6}

\section{Anxiety and depression following preeclampsia or HELLP syndrome. A systematic review}

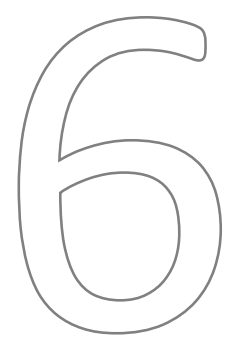

Denise HJ Delahaije, Carmen D Dirksen, Louis L Peeters, Luc J Smits

Acta Obstet Gynecol Scand, 2013, 92:746-61 


\begin{abstract}
Background

Women who suffered from pregnancy complications are at increased risk for anxiety and depression.

Objective

To evaluate whether having suffered from preeclampsia or HELLP syndrome is associated with anxiety and depression, and whether preeclampsia/HELLP is an independent risk factor for developing anxiety and depression.
\end{abstract}

\title{
Search strategy
}

Systematic search on PubMed and Psyclnfo with no time limit.

\section{Selection criteria}

Studies presenting original data, including women with a history of preeclampsia/HELLP and at least one comparison group of women without preeclampsia/HELLP, reporting the results for each group separately or in a multivariate regression analysis with preeclampsia/HELLP as an independent variable.

Data collection and analysis

Study characteristics and outcomes were extracted using a prespecified form. If necessary, additional calculations were performed.

\section{Results}

The search yielded 267 articles, with only six being suitable for inclusion in this review. Studies on depression (6) showed generally positive associations between preeclampsia/HELLP and the prevalence of depression or severity of depressive symptoms. However, the results of three studies were not statistically significant. Studies addressing anxiety (2) did not show significant associations between preeclampsia/HELLP and anxiety scores. Associations between posttraumatic stress and preeclampsia/HELLP, investigated in four studies, were often non-significant. Due to heterogeneity of study methods, a meta-analysis of the results was not possible. In most studies, confounder control was poor.

\section{Conclusions}

Evidence is mixed but generally points to positive associations between various forms of psychopathology and previous preeclampsia/HELLP. Causality of the associations can, however, not be judged adequately. 


\section{Introduction}

Traditionally, pregnancy is considered a period of well-being and happiness, and was once thought to protect women from anxiety and depression (1, 2). However, $20 \%$ of women experience moderate depression during pregnancy and almost $13 \%$ suffer from major depressive disorder during the first year postpartum $(3,4)$. Elevated anxiety levels are found in one out of seven women 30 weeks postpartum (5). Women who suffered from pregnancy complications are at higher risk for anxiety and depression (68). During pregnancy and the perinatal period, women are more likely to be exposed to stressful situations, such as maternal complications, preterm delivery, fetal distress, emergency cesarean section, and maternal or neonatal admission to intensive care.

Preeclampsia (PE) and the hemolysis, elevated liver enzymes, and low platelets (HELLP) syndrome are common pregnancy complications, together affecting about 3\% of all pregnancies. To date, it is unknown whether and to what extent these complications predispose to anxiety and depression. A better understanding of the occurrence and severity of anxiety and depression in former PE or HELLP patients may lead to improved preventive strategies and targeted interventions. Prevention and timely recognition of these anxiety and depression can reduce treatment duration and costs (9). Untreated anxiety and depression can persist for years and can adversely affect infants (10-13). Currently, the identification and management of anxiety and depression during pregnancy and the perinatal period are still limited (14). Some studies addressing anxiety and depression in women with (a history of) PE or HELLP reported increased rates of depression, anxiety, or posttraumatic stress disorder (PTSD) during the first months postpartum $(15,16)$. Other studies, however, failed to substantiate these findings (17). Differences in study outcomes may be contributable to differences in the designs of these studies and the composition and sizes of the populations enrolled.

In this article we reviewed the existing literature with respect to two questions. The first question was whether former PE/HELLP patients are more likely to have anxiety and depression or more severe anxiety and depression, as compared to women without a history of PE/HELLP. The second question was whether PE/HELLP is an independent risk factor for developing anxiety and depression. If anxiety and depression do occur more often in former PE/HELLP women than in their non-PE/HELLP counterparts, differences with respect to other determinants of anxiety and depression may be part of the explanation. For this purpose we used the term "anxiety and depression" to refer to depressive symptoms, diagnosis of depression, anxiety symptoms, diagnosis of anxiety, posttraumatic stress symptoms and posttraumatic stress disorder. Posttraumatic stress disorder is a specific form of anxiety that can be triggered by experiencing a traumatic, life-threatening event. 


\section{Material and methods}

\section{Study design/Search strategy}

We systematically reviewed original articles reporting on the occurrence or severity of anxiety and depression in women who experienced PE or HELLP as well as women who did not experience PE or HELLP during their pregnancy. The study was completed in line with the Preferred Reporting Items for Systematic Reviews and Meta-Analyses (PRISMA) guidelines (18). A literature search was conducted in PubMed and Psyclnfo in March 2013 in order to identify eligible articles. The search was performed with no time limit applied to any database. Aiming for a sensitive search strategy, we extended the literature search beyond preeclampsia and the HELLP syndrome and also used toxemia, pregnancy-induced hypertension, hypertensive disorders of pregnancy and hypertensive disorders in pregnancy as search terms.

We searched for combinations of search terms in title or abstract by using the following free text words: exposure: 'preeclamp*' OR 'pre-eclamp*' OR 'pregnancyinduced hypertension' OR 'pregnancy induced hypertension' OR 'hypertensive disorders of pregnancy' OR 'hypertensive disorders in pregnancy' OR 'toxaemia' OR 'toxemia' OR 'hellp' OR 'hemolysis elevated liver enzymes and low platelets' and outcomes: 'depress*' OR 'anxiet*' OR 'posttraum*' OR 'post-traum*' OR 'panic' OR 'PTSD' OR 'GAD'.

The search was limited to studies published in English and included an abstract. The search resulted in a total of 267 articles. The following criteria were used to determine whether a study was relevant for the review: 1) the study had to present original data; 2) the study had to consider postpartum depression, anxiety, or posttraumatic stress as an outcome; 3 ) the study had to include both women with a history of PE/HELLP and a comparison group of women who had not experienced PE or HELLP; 4) the study had to either present the results for each group separately, or present the results of a multivariate regression analysis in which the diagnosis of PE/HELLP was considered as an independent factor, or both.

Retrieved records were collected in alphabetical order. Duplicates were removed and non-relevant titles were excluded. The main reason for excluding articles was that these studies involved topics or subjects that were not related to the purpose of this review. Abstracts were obtained of all potentially eligible articles that remained after the title screening. Two reviewers (D.D. and C.D.) independently assessed the relevance of the remaining abstracts. Any discrepancies regarding inclusion or exclusion of a study were resolved by reading the full article and discussion. Both reviewers excluded 14 abstracts. One reviewer (D.D.) read all remaining articles in full, while a second 
reviewer was consulted (C.D.). We also conducted a hand search of the reference list of the eligible articles to identify additional studies.

\section{Data extraction and quality aspects}

Data extraction was performed by one of the authors (D.D.). Extracted data were checked for completeness and correctness by the second author (C.D.). Any disagreements were resolved by either discussion or by consulting a third reviewer (L.S.). For each paper, the reviewers extracted basic study characteristics with respect to authorship, year of publication, research question/aim, study design (such as type of study, inclusion moment), study population (such as patient characteristics, sample size), outcome(s) of interest, data collection method and instruments. Moreover, the findings of interest for the current review were extracted.

A short critical appraisal checklist was used in order to facilitate data extraction and evaluate the appropriateness of the studies in light of our review questions. This evaluation was not used to assign weights or exclusion criteria, but rather as a subject for discussion. The checklist was adapted from the Newcastle-Ottawa Quality Assessment Scale for cohort studies (19). This scale has established content validity and interrater reliability (19). The individual components of the checklist were rated according to a $\mathrm{Y} / \mathrm{N} /$ ? system rather than according to the allocation of stars per rating sheet. With the checklist we assessed whether the non exposed cohort was drawn from the same community as the exposed cohort, whether the exposure was ascertained by a physician, how the outcome was assessed (such as medical records, self-report), whether a non-response analysis was performed, whether the recall period was longer than implicated in the questionnaires and whether the same follow-up period was applied for all patients.

\section{Confounder control}

For the purpose of the second review question (i.e. whether PE/HELLP is an independent risk factor for developing anxiety and depression), confounder control and handling of intermediate variables were specifically considered important. Treating intermediates as confounders is not appropriate as it may introduce, rather than control for, bias in the association between PE/HELLP and anxiety and depression. Further elaboration on handling of potential confounders and intermediate factors can be found elsewhere (20).

Based on the literature, we considered the following factors potentially important confounders in the association between PE/HELLP and anxiety and depression: maternal obesity (21-23) , low socioeconomic status (24-27), previous mental illness (28-30), 
pre-existing medical conditions $(27,31,32)$, age $(27,33,34)$, parity $(35-37)$ and nonWestern ethnicity (38-40). These factors are risk factors of anxiety and depression and are related to PE/HELLP. Ideally, these factors should be treated as potential confounders in each study. Other risk factors for anxiety and depression may be on the causal path between PE/HELLP and anxiety and depression and should therefore not be treated as confounders. These factors include mode of delivery, gestational age, birthweight, suspicion of fetal distress, Apgar score, perinatal death, hospital admission of mother or child and anxiety and depression during pregnancy.

\section{Results}

Figure 1 summarizes the literature identification and selection procedure. Of the 267 articles identified in the PubMed/Psyclnfo search, six articles ultimately met the inclusion criteria, i.e. Engelhard et al., 2002 (16); Brussé et al., 2008 (17); Baecke et al., 2009 (41); Blom et al., 2010 (15); Gaugler-Senden et al., 2011 (42); Stramrood et al., 2011 (43). The reference check did not reveal any additional relevant articles.

Table 1 lists the main characteristics of all studies included in the systematic review. The selected articles comprised four retrospective cohort studies and two prospective cohort studies. All studies were performed in the Netherlands. Sample sizes ranged from 20 to 4941, with a total of 5636 women included. One author participated in two different studies but inclusion of patients in these studies took place in different time periods $(16,41)$. One author participated in three different studies and we could not rule out a possible overlap of patients between these studies because inclusion of patients in these studies took place in the same period and in the same hospital (15, 17, 42). Therefore, we contacted this author who assured us that there was no such overlap. Two studies included two groups, of which one included severe PE patients and women with uncomplicated normotensive pregnancies (17), and the other included severe, early-onset PE/HELLP patients with preterm delivery and healthy participants with a preterm delivery (42). One study included three groups, PE patients, patients with preterm premature rupture of membranes and women with uncomplicated pregnancies (43). Two studies each included four groups, patients with preterm PE, patients with term $P E$, patients with preterm birth and patients with an uneventful pregnancy $(15,16,41)$. Blom et al. (15) included a general sample of pregnant women and examined a wide range of perinatal complications (among which was PE) as potential risk factors for postpartum depression. All selected studies reported on depression, four studies reported on PTSD $(16,41-43)$ and two studies reported on anxiety (17, 41). 


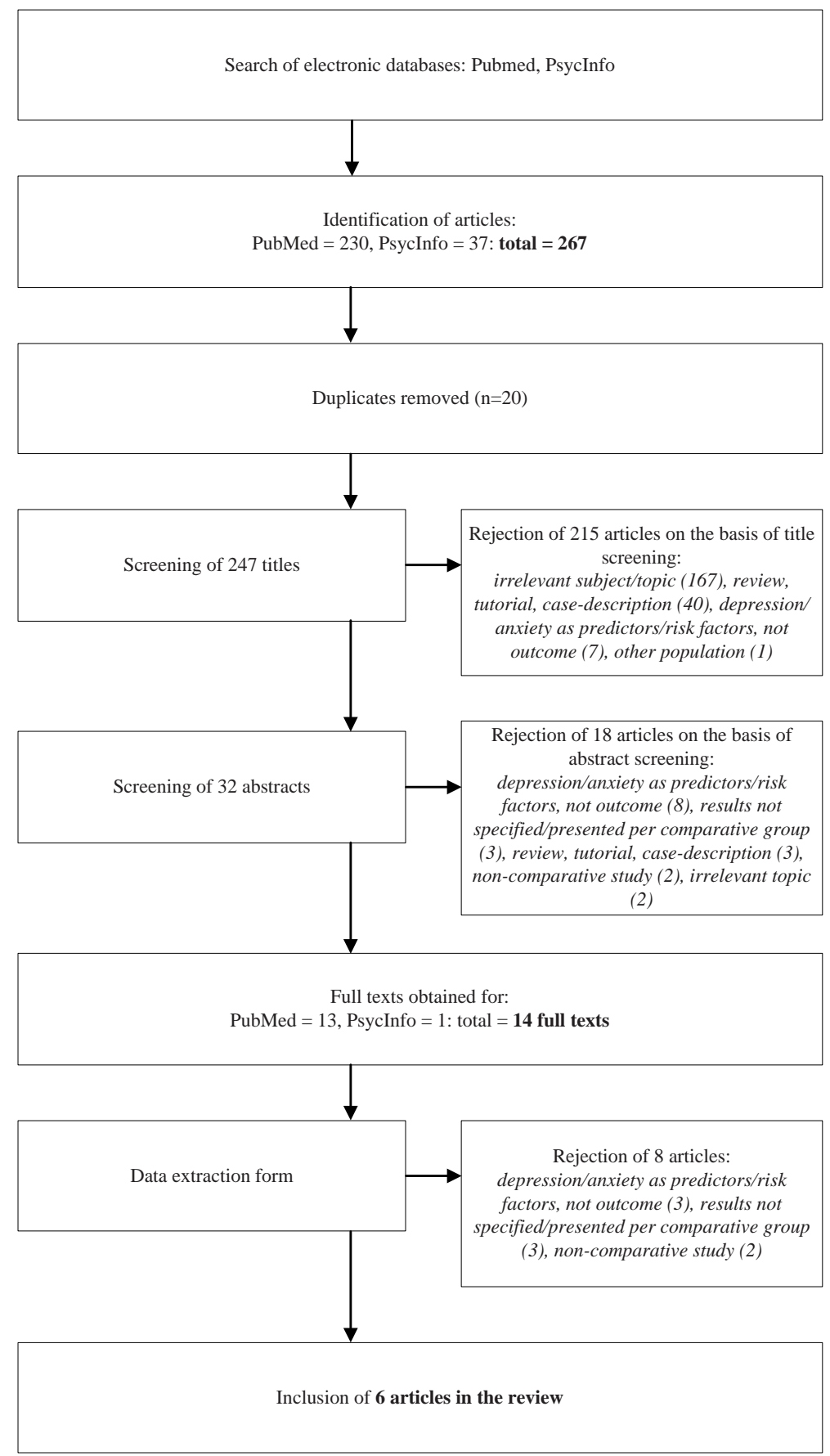

Figure 1: Study selection process 


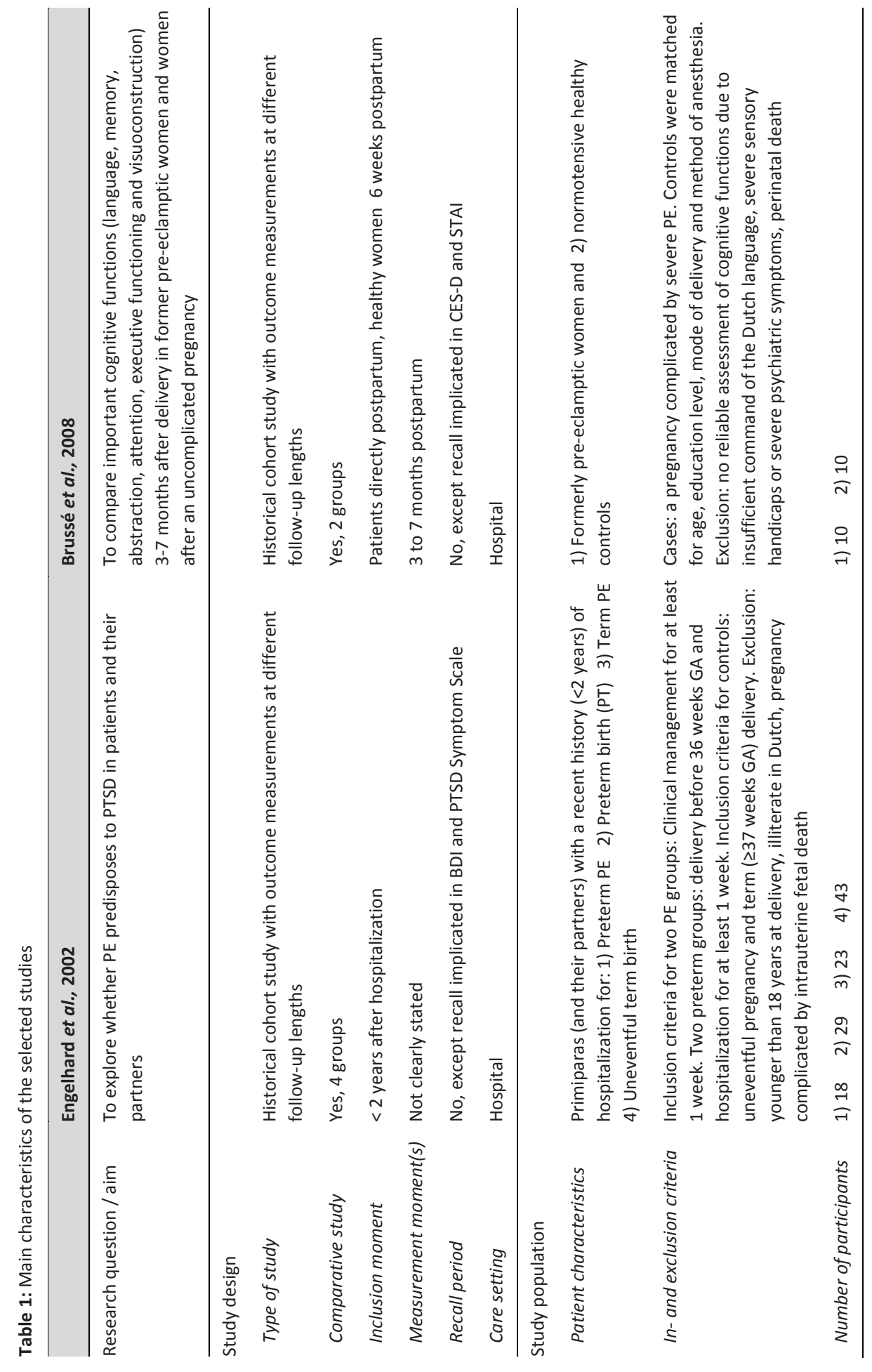




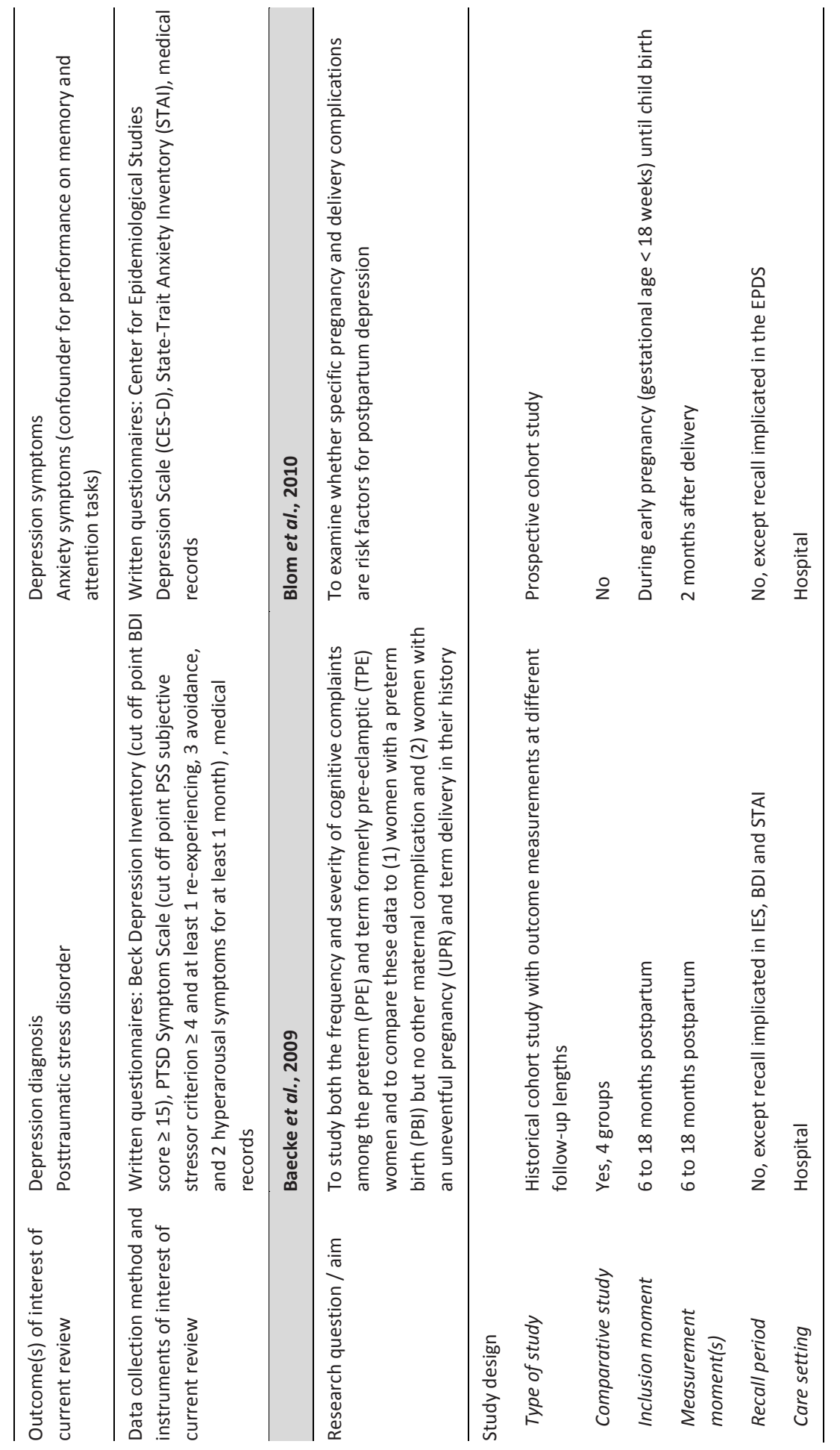




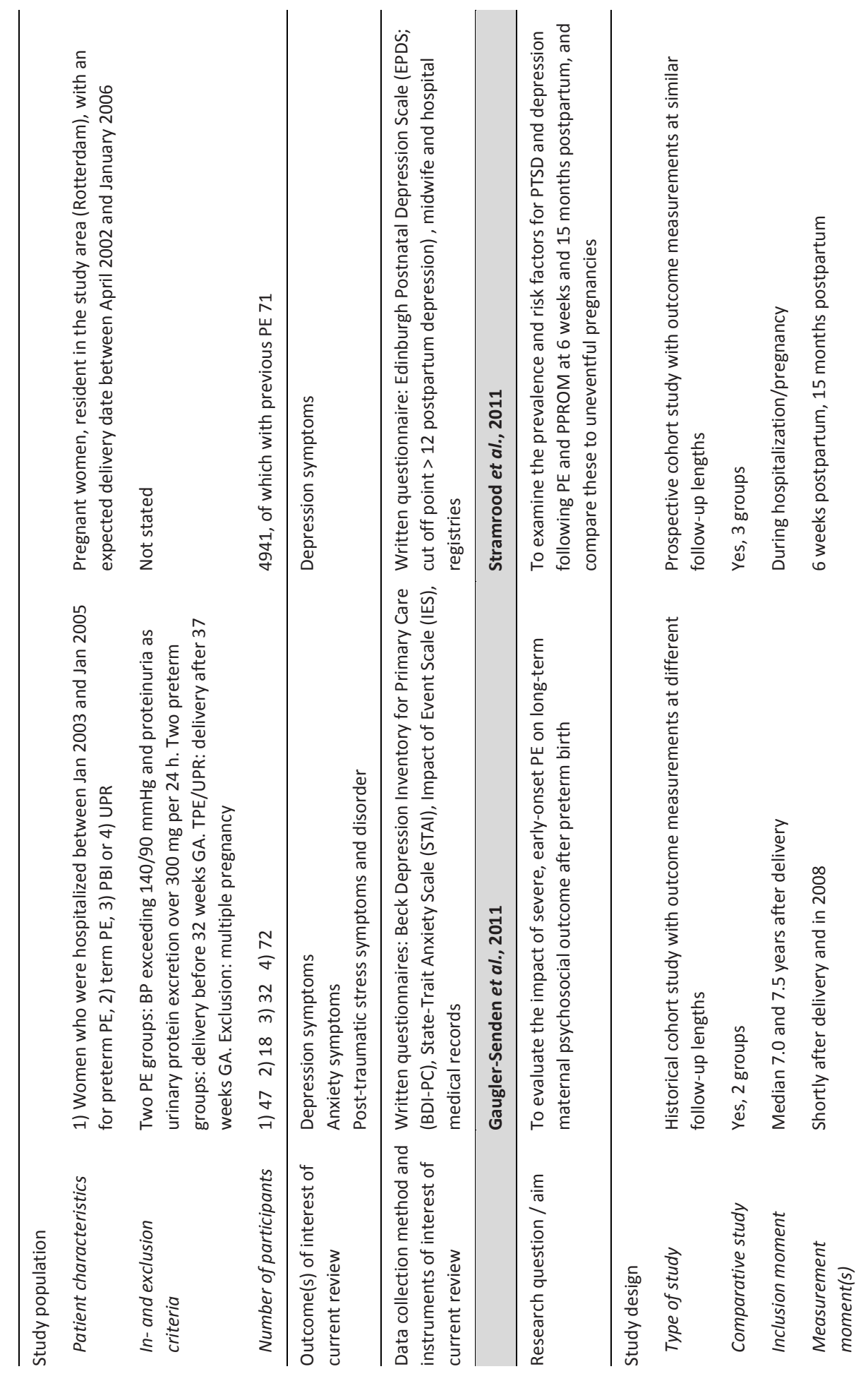




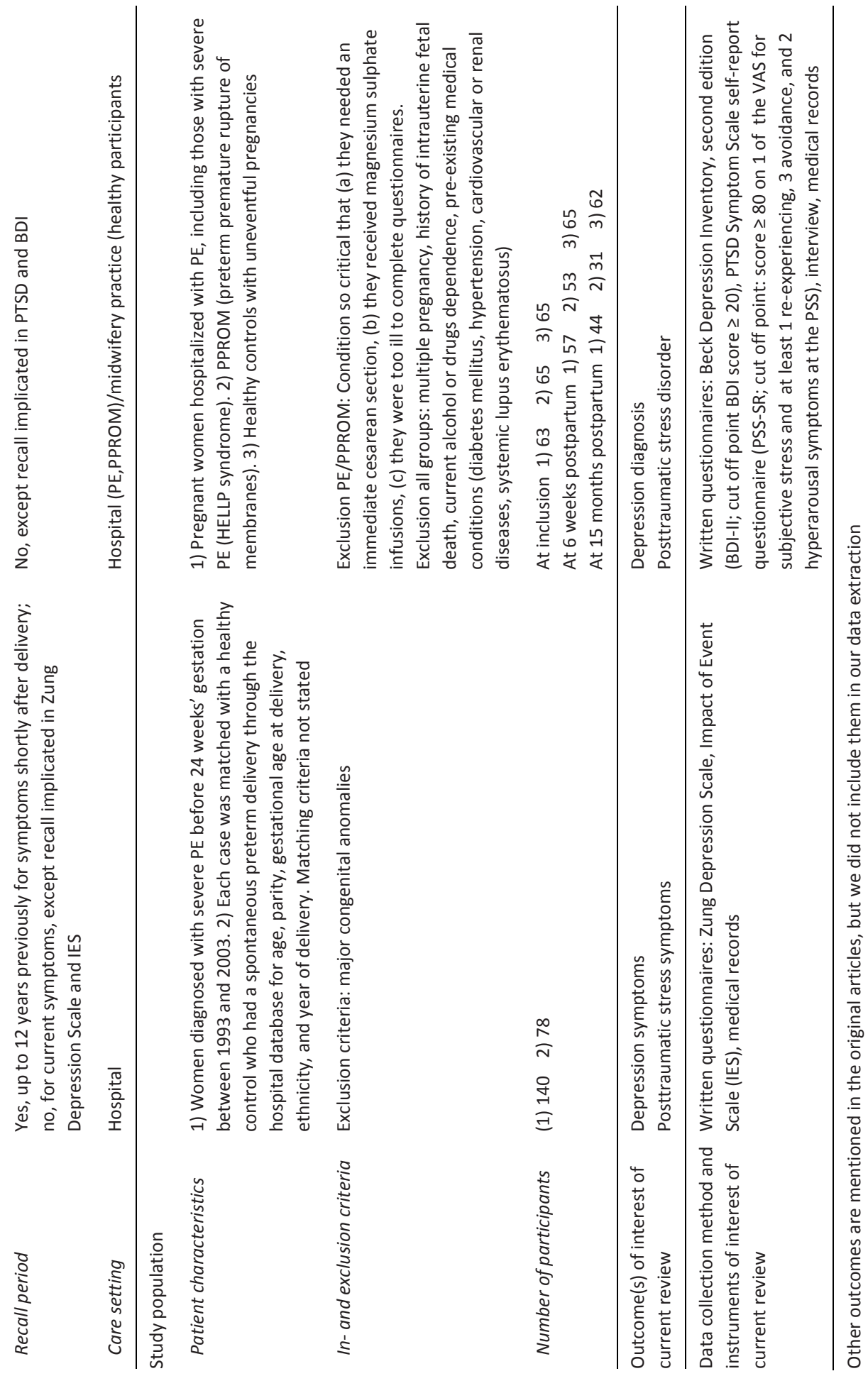




\section{Quality aspects}

Index cases and control women were recruited from the same source population in all studies but one. Stramrood et al. (43) recruited pregnant women with PE or HELLP in the hospital, whereas healthy controls with uneventful pregnancies were recruited in an independent midwifery practice. All the other studies recruited both PE or HELLP patients and comparison group(s) from the same source, i.e. the hospital.

In all studies, exposure (PE/HELLP) was ascertained by a physician. Outcome assessment was done using validated questionnaires in all studies. Recall periods were no longer than implicated in the questionnaires used in all studies but one. In the study of Gaugler-Senden et al. (42) the recall period varied from four up to 12 years. Timing of assessment was equal for all patients in only two studies $(15,43)$.

Blom et al. (15) performed a non-response analysis showing that women with missing data reported more psychopathological symptoms, poorer family functioning, had a lower family income, were less educated, were more likely to be of non-Western origin and younger than responders. Engelhard et al. (16) and Gaugler-Senden et al. (42) reported response rates of the different groups and showed no significant differences between respondents and non-respondents with regard to age, parity, gestational age at delivery, prolongation of pregnancy or year of delivery. Baecke et al. (41) reported response rates of the different groups, but did not analyze differences between responders and non-responders. Brussé et al. (17) did neither report response rates, nor investigate differences between responders and non-responders. Stramrood et al. (43) included 193 women, whereas at the end of the study, only 137 participants were left. There may be selective drop-out since women who did not take part at the end of the study had a slightly lower educational level and were younger than participants.

\section{Reported outcomes of the selected studies}

The most frequently studied outcome was depression, followed by posttraumatic stress and anxiety. Concerning these outcomes, a distinction was made between occurrence and severity. Four studies used cut-off points for the questionnaires to measure clinical levels of the outcomes, i.e. diagnoses $(15,16,41,43)$.

\section{Review question 1}

The following sections address the first review question, i.e. whether anxiety and depression occur more frequently or are more severe after PE/HELLP than after a pregnancy not complicated by PE/HELLP. Table 2 summarizes the results of the studies. For the purpose of the first review question, it is important that a study allows a separate comparison of women with PE/HELLP to women without PE/HELLP. 


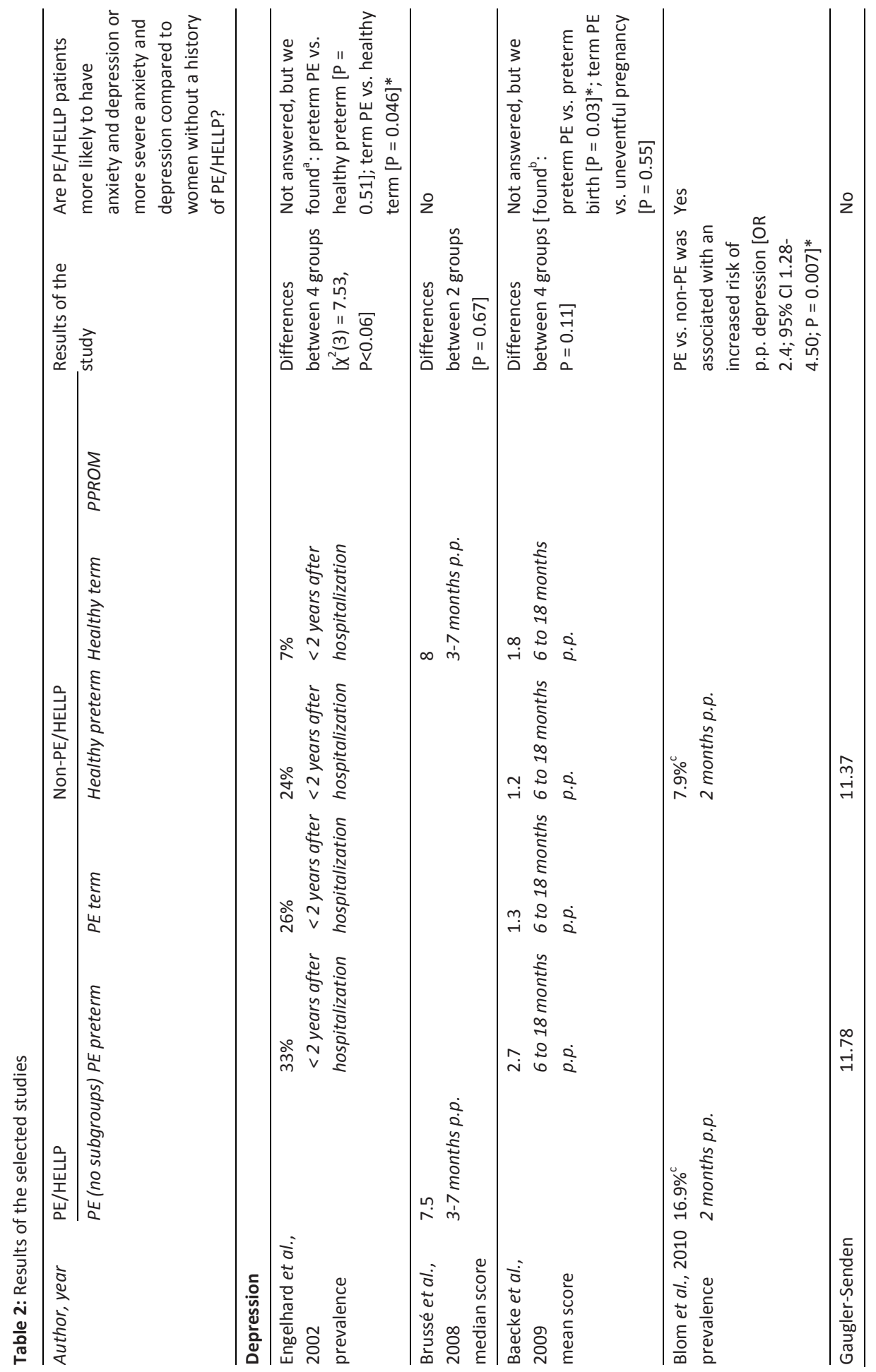




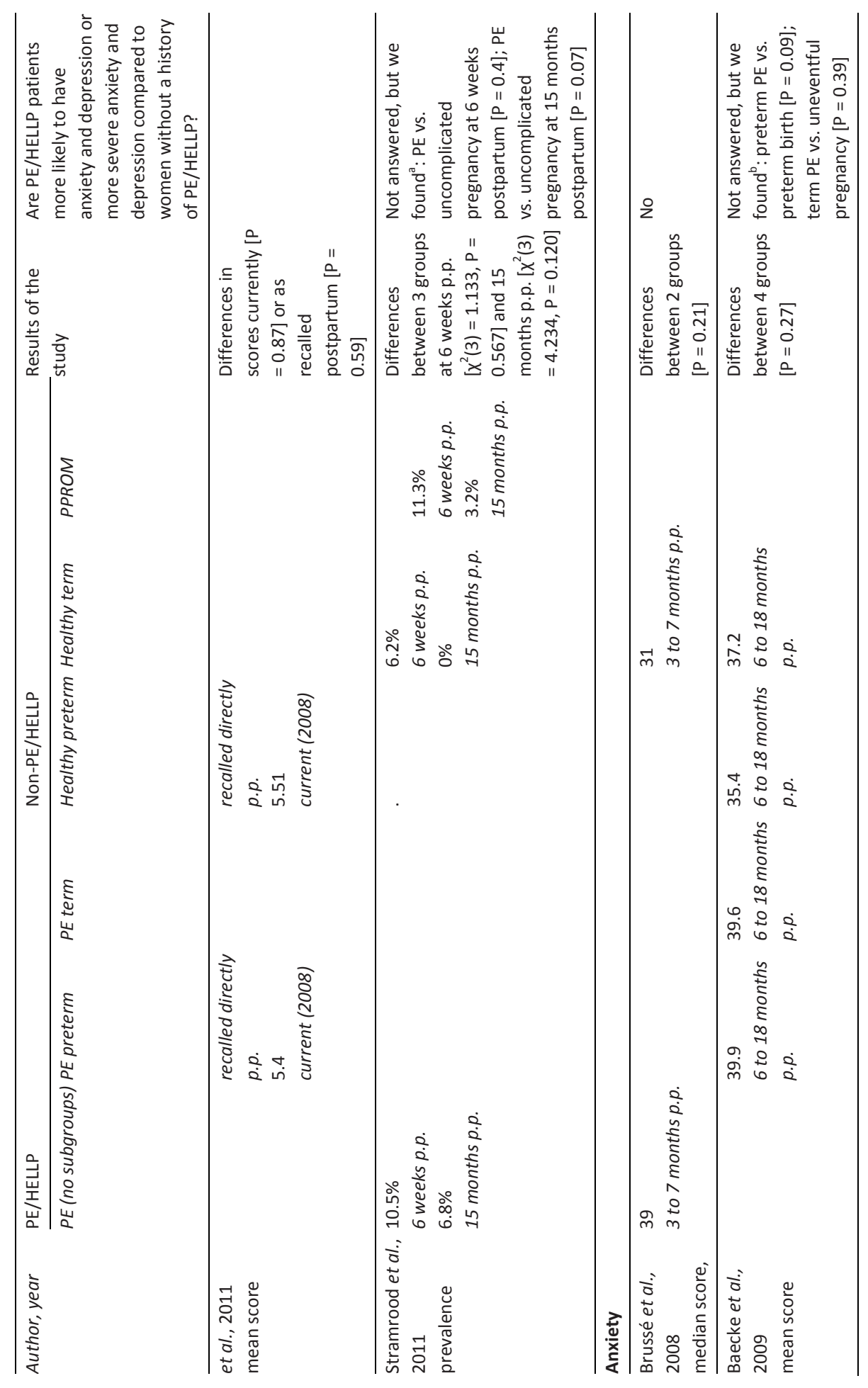




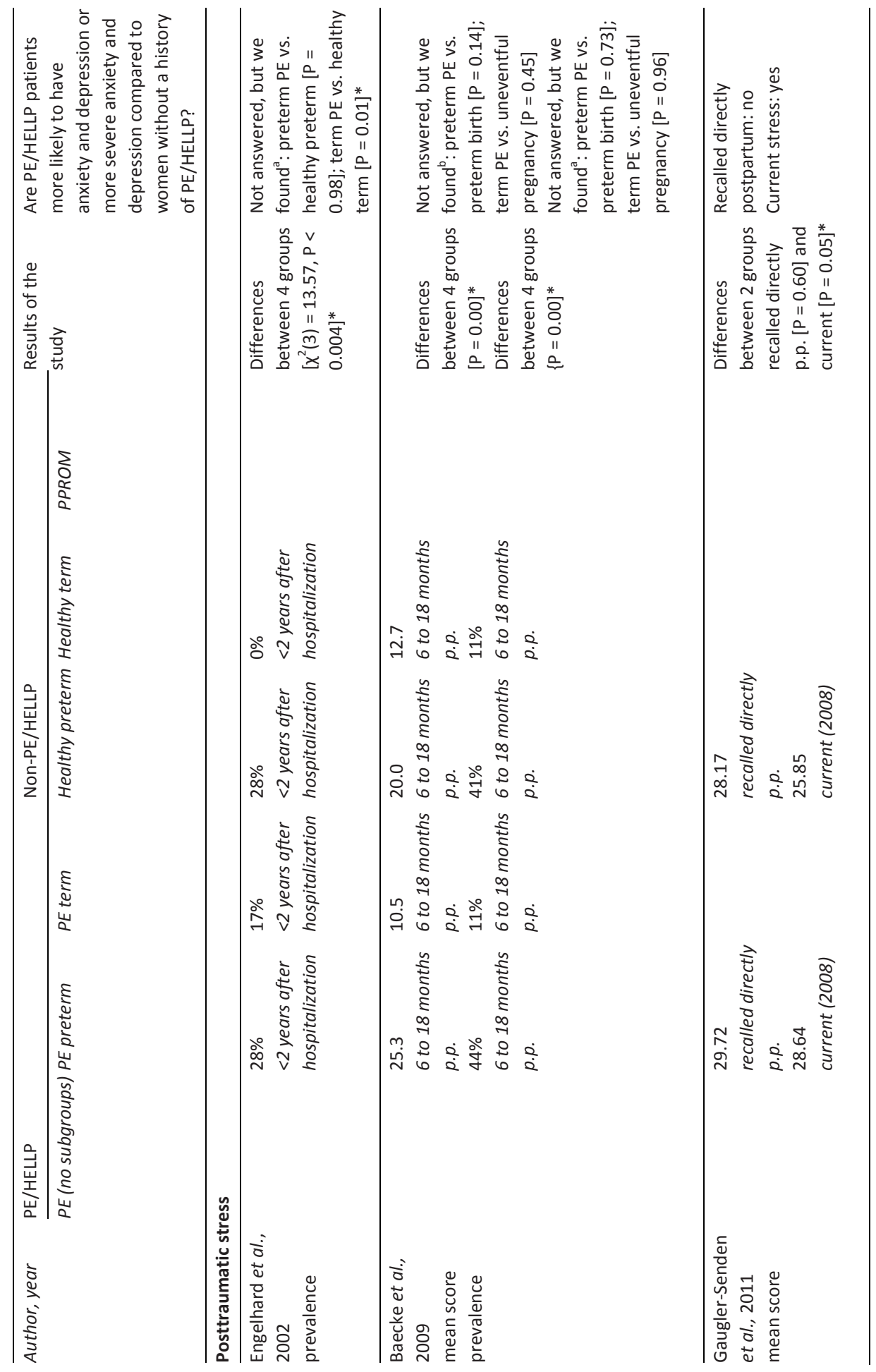




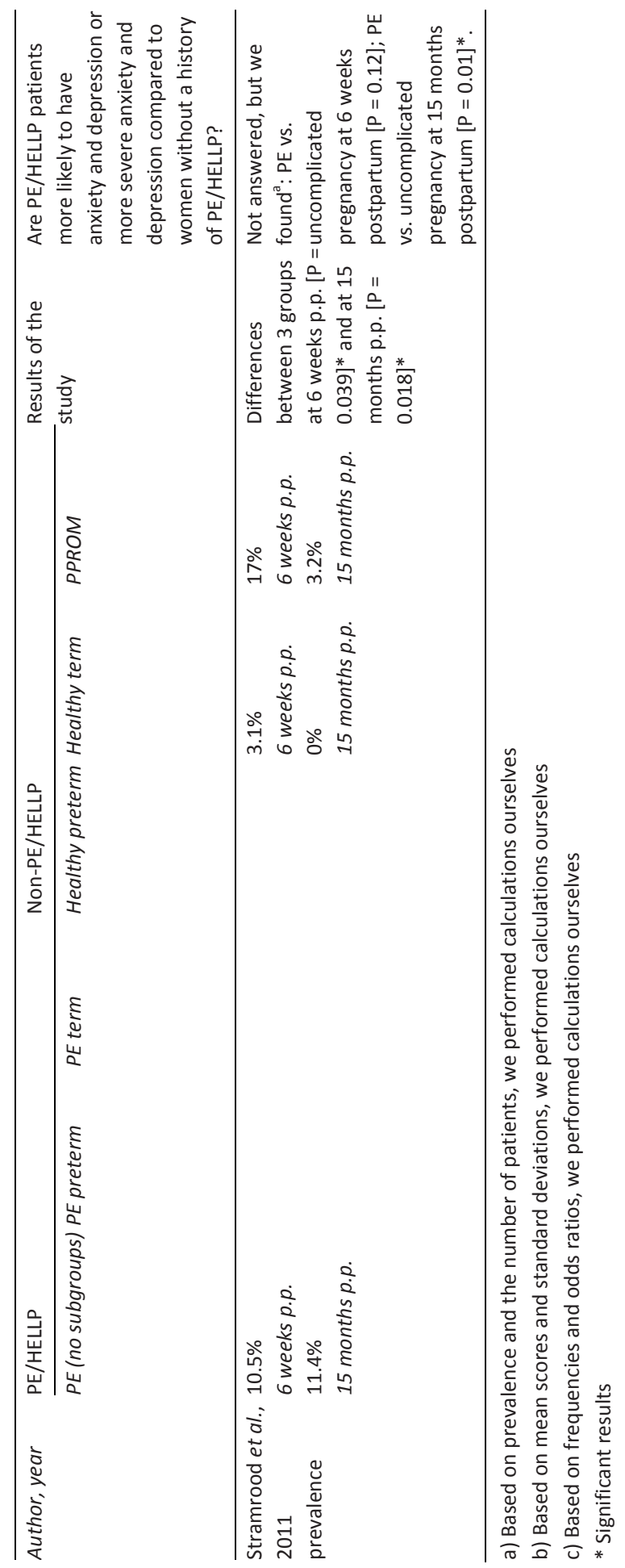




\section{Depression}

Three studies used the Beck Depression Inventory (BDI) $(16,41,43)$, one study used the Zung Depression Scale (42), one study used the Edinburgh Postnatal Depression Scale (EPDS) (15) and one study used the Center for Epidemiological Studies Depression Scale (CES-D) (17) for the measurement of depression. Three studies measured severity $(17,41,42)$ and three studies measured occurrence $(15,16,43)$. Most of the studies showed positive associations between PE/HELLP and the prevalence of depression and severity of depressive symptoms. All studies but one reported no significant differences in occurrence or severity of depression among the groups $(16,17,41-43)$. Only three studies reported results in such a way that review question 1 could be answered directly $(15,17,42)$, of which one measured prevalence $(15)$ and two severity $(17,42)$. The studies including three or more groups did not separately compare women with PE/HELLP to women without PE/HELLP and are therefore not directly useful to answer our first review question $(16,41,43)$. However, we performed calculations ourselves in order to answer review question 1 with these studies. Using the data reported in the study of Engelhard et al. (16), we found a significant difference in the occurrence of depression between term PE and term patients without PE, and we found no significant difference between preterm PE patients and patients delivering preterm without PE. The study of Brussé et al. (17) reported no significant differences in the severity of depression between PE patients and normotensive healthy controls. With the study of Baecke et al. (41), we found a significant difference in the severity of depression between preterm PE and healthy preterm patients and no significant difference between term PE and healthy term women. The study of Blom et al. (15) reported that PE was significantly associated with an increased risk of postpartum depression. The study of Gaugler-Senden et al. (42) reported no significant differences in the severity of depression between preterm PE/HELLP and healthy preterm patients, neither current, nor recalled directly postpartum. Using the data of Stramrood et al. (43), we found no significant differences in the occurrence of depression between PE/HELLP and healthy pregnant women, neither six weeks postpartum, nor 15 months postpartum.

\section{Anxiety symptoms}

Two studies used the State-Trate Anxiety Inventory (STAI) for the measurement of anxiety symptoms $(17,41)$ and only severity was measured, since the STAI is not a diagnostic instrument. Both studies showed higher anxiety severity scores among women with $\mathrm{PE}$, however, these differences were not significant. Only one study reported results in such a way that review question 1 could be answered directly. The study of Brussé et al. (17) reported no significant differences between PE patients and the healthy controls regarding the severity of anxiety symptoms. The study of Baecke 
et al. (41) included more than three groups but did not separately compare women with PE to women without PE. However, we performed calculations ourselves and found no significant differences in the severity of anxiety between preterm PE patients versus healthy preterm patients and term PE patients versus healthy term patients.

\section{Posttraumatic stress}

Two studies used the Impact of Event Scale (IES) $(41,42)$ and two other studies used the PTSD Symptom Scale $(16,43)$ for the measurement of posttraumatic stress. Two studies measured severity $(41,42)$ and three studies measured occurrence $(16,41,43)$. In general, women with PE/HELLP have higher prevalence and severity rates of posttraumatic stress than their counterparts.

All studies showed significantly different levels or a significantly different prevalence of posttraumatic stress among the groups $(16,41-43)$, but only two reported results in such a way that review question 1 could be answered directly $(41,42)$. Again, we performed calculations ourselves in order to answer the first review question. With the study of Engelhard et al. (16), we found a significant difference in the occurrence of PTSD between term PE and healthy term patients and no significant difference between preterm PE and healthy preterm patients. With the study of Baecke et al. (41), we found no significant differences in the severity and occurrence of posttraumatic stress between preterm PE versus healthy preterm patients and term PE versus healthy term patients. The study of Gaugler-Senden et al. (42) reported no significant differences as recalled directly postpartum in the severity of posttraumatic stress between preterm PE/HELLP and healthy preterm patients. However, it reported significant differences in current severity of posttraumatic stress between the two groups.

Reanalyzing the data of the study of Stramrood et al. (43), we found no significant difference in the occurrence of PTSD at six weeks postpartum between PE/HELLP patients and women with an uncomplicated pregnancy. However, there was a significant difference in the occurrence of PTSD at 15 months postpartum between the two groups.

\section{Review question 2}

Table 3 describes different strategies applied in the studies to control for confounding. The strategies for confounder control differed between studies as did the selection of confounders. Restriction was applied in five of the six studies. Three studies unfortunately restricted for potential intermediates such as fetal death $(16,17,43)$. Two studies conducted matching $(17,43)$, however, matching categories within variables were not made explicit in both studies, and potential intermediates such as mode of delivery (17) and gestational age (42) were among the matching factors. Three studies con- 
trolled for possible covariates in a multivariate analysis $(15,16,43)$. However, all studies adjusted for potential confounders as well as potential intermediate factors. None of the studies controlled for maternal BMI, and only two controlled for previous mental illness $(17,43)$.

The second review question, i.e. whether previous PE/HELLP is an independent risk factor of subsequent anxiety and depression, was considered to be answered if a study controlled for at least four possible confounders (as defined previously) and ideally, did not control for intermediates. Since controlling for intermediate factors and insufficient control for confounders can bias associations towards the null as well as away from it, Blom et al. (15) provides the most valid evidence with respect to our second review question. The study of Engelhard et al. (16) controlled for only two previously defined confounders and for too many intermediates; Brussé et al. (17) controlled for only three previously defined confounders, but fortunately, did control for only a few intermediates; Baecke et al. (41) controlled for no previously defined confounders; Gaugler-Senden et al. (42) controlled for three previously defined confounders, and controlled for one intermediate. Brussé et al. (17) excluded patients when a reliable assessment of cognitive functions could not be made due to severe psychiatric symptoms. However, they did not define severe psychiatric symptoms and therefore, it is unclear whether they were present before the development of PE. Blom et al. (15) adjusted for general psychopathological symptoms as a covariate, however, they just stated that general psychopathological symptoms included at least depression and anxiety. Again, it is unclear whether they were present before the development of PE. Treating severe psychiatric symptoms and general psychological symptoms (15) and severe psychiatric symptoms (17) as confounders is only appropriate in case they were present before the development of PE, which was not reported. Therefore, 'severe psychiatric symptoms' and 'general psychological symptoms' are described as 'other factors' instead of confounders in Table 3. 


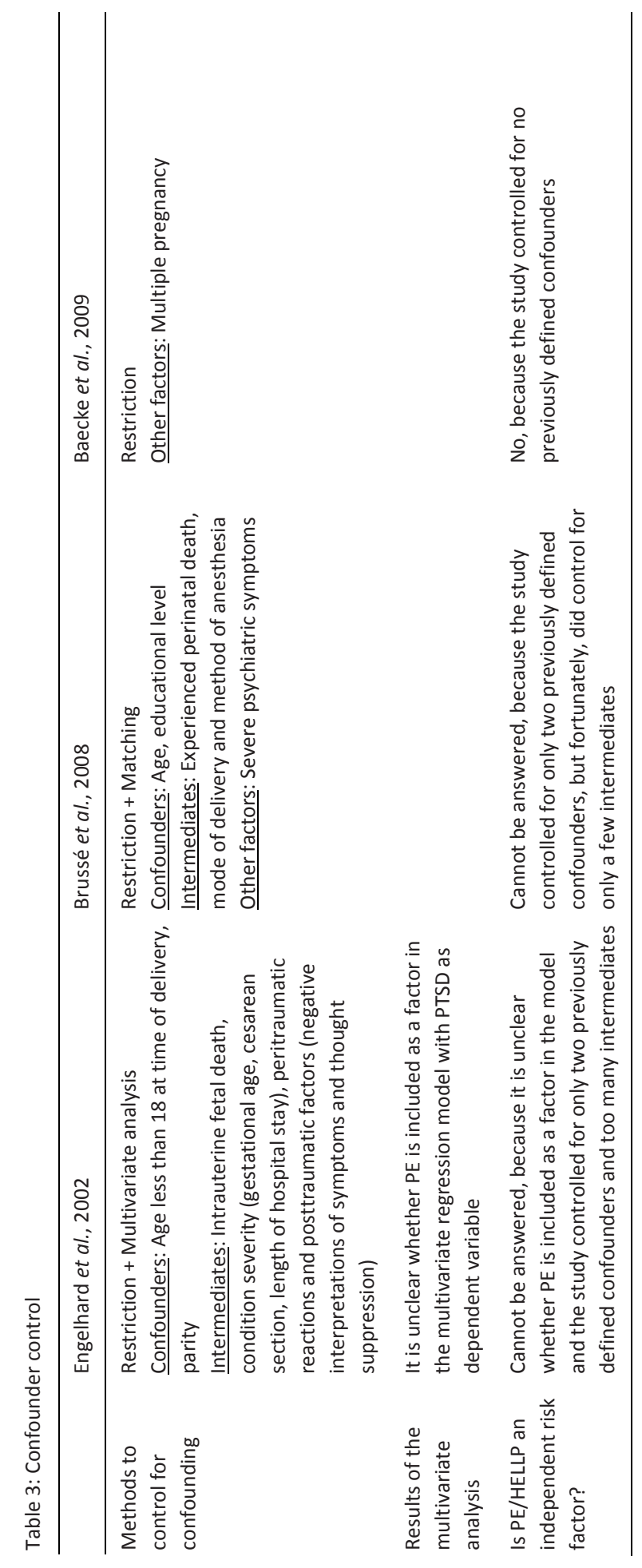




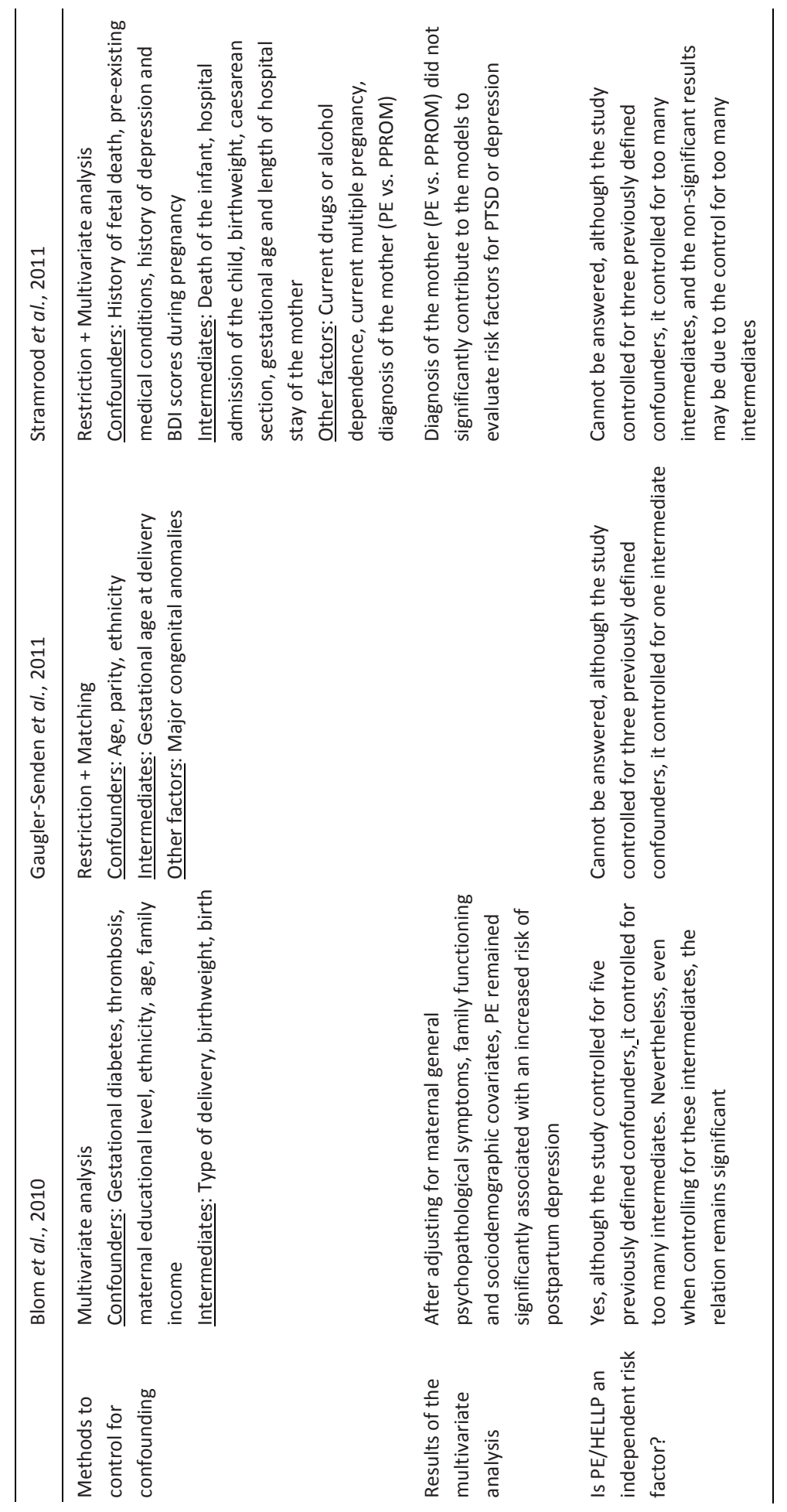




\section{Discussion}

We have summarized the available evidence about the incidence and occurrence of anxiety and depression in patients who experienced a pregnancy complicated by PE or HELLP and put the evidence in a methodological context. We identified six cohort studies (four historical and two prospective) examining occurrence or severity of anxiety and depression in women who developed PE or HELLP syndrome during pregnancy compared to women who did not experience any of these pregnancy complications.

Our first review question was whether former PE/HELLP patients are more likely to have anxiety and depression or more severe anxiety and depression, compared to women without a history of PE/HELLP. With respect to depression, the evidence is mixed. Although nearly all studies showed positive associations between PE/HELLP and prevalence of depression or severity of depressive symptoms, the results of three of them were not statistically significant $(17,42,43)$. Of the two studies distinguishing between term and preterm deliveries, one showed an increased prevalence among women with PE and term births (depression prevalence $26 \%$ versus $7 \%$ of healthy term women; PTSD prevalence $17 \%$ versus $0 \%$ of healthy term women) (16), whereas the other showed increased severity among women with PE and preterm deliveries (depression severity of 2.7 vs. 1.2 of healthy preterm women; anxiety severity 39.9 vs. 35.4 of healthy preterm women; PTSD prevalence $44 \%$ vs. $41 \%$ of healthy preterm women; PTSD severity 25.3 vs. 20.0 of healthy preterm women) (41). The two studies addressing anxiety showed no significant associations between PE and anxiety scores, although differences were in the expected direction, i.e. higher scores among women with PE $(17,41)$. In the four studies addressing posttraumatic stress, associations were generally in the expected direction, i.e. higher prevalence and severity among women with PE/HELLP, and three of them were partly significant $(16,42,43)$. The results of two studies indicate that the posttraumatic stress symptoms may increase over time $(42,43)$.

Our second review question was whether PE/HELLP is an independent risk factor of anxiety and depression. In most studies confounder control was poor. Some studies treated potential intermediate factors as confounders, which may lead to underestimation of effects. One study, addressing the occurrence of depression, controlled for a number of potential confounders and intermediate factors and still found a statistically significant association with PE. This indicates that PE/HELLP may be an independent risk factor of subsequent depression (15).

It can be argued that non-significant results are not a problem since it can be solved by pooling the data. However, we could not perform a meta-analysis because comparability of studies was poor due to different subgroups, different outcomes 
(occurrence and severity), different questionnaires, different cut off points and different follow-up lengths.

Although in all studies validated questionnaires for outcome assessment were used, only in two were they submitted to patients at fixed time points after the pregnancy affected by PE or HELLP. As prevalence and severity of anxiety and depression may increase or decrease as a function of time after delivery, interpretation of the results of studies not using fixed assessment points is complicated. Studies used different questionnaires to screen for anxiety and depression with different validity and reliability $(4,44)$. Although PSE/SCAN or SCID interviews would be the gold standard for measurements in psychopathology, they are very time-consuming and therefore not appropriate in clinical studies in which many patients are included. It has been found that self-report measures are fairly accurate in assessing emotions and behaviors. Within the research context, if large-scale screening does occur, it is more timeefficient to conduct clinical interviews with only those patients scoring above the cutoff score (44). Only three instruments (Bromley Postnatal Depression Scale, Edinburgh Postnatal Depression Scale, Postpartum Depression Screening Scale) have been developed specifically to measure postpartum depression, whereas other instruments measure general symptoms of depression. Reviews of screening instruments for postpartum depression found that the EPDS, the BDI-II and the PDSS have greater sensitivity and specificity in the perinatal population than other measures that have been tested (45). There is no literature concerning the optimal choice of measurement instruments for anxiety and posttraumatic stress in perinatal settings.

Furthermore, one study asked participants to recall their mental health status over a longer period than implicated in the questionnaire (up to 12 years), which is likely to have led to considerable misclassification (42).

All included studies were performed in the Netherlands among predominantly Caucasian women. Results may therefore not be applicable to non-Caucasian women. Although most studies mentioned the number of women unwilling to participate in the study (response rates ranging from 48-90\%), most did not present data comparing characteristics of respondents and non-respondents. Therefore it is not possible to evaluate representativeness of the study population. Study participation may be associated with mental health.

Depression and anxiety disorders have been identified as risk factors of PE (29, 46). Furthermore, the risk of postpartum depression is strongly associated with prepregnancy psychopathology (47). Therefore, controlling for pre-pregnancy mental health is essential in unraveling the causal relation between PE and psychopathology. Most of the studies included in this review did not obtain measures of maternal mental health status before the diagnosis of PE or HELLP was made $(15,16,41-43)$. Blom et al. (15) adjusted for general psychopathological symptoms as a covariate, but mentioned 
that these symptoms were measured at 30 weeks of gestation which may be after the diagnosis of PE. Stramrood et al. (43) measured depression and BDI scores after the diagnosis of PE or HELLP was made; thus, part of the psychopathology might have been caused by the pregnancy complication and controlling for it may be unjustified.

\section{Conclusions and implications for further research}

Evidence about the association of PE/HELLP with subsequent psychopathology is mixed. Differences between study methodologies are likely to have contributed to the heterogeneity of the results. Nevertheless, associations that were found were generally in the same direction, i.e. towards higher prevalence/severity of psychopathology in women with previous PE/HELLP, in comparison to women with no such history. In order to be able to better evaluate causality of the associations, evidence is needed from studies controlling for important potential confounders including psychopathology at baseline. Such studies should preferably be prospective and have outcome measurements at fixed time points.

\section{References}

1. Qiu C, Sanchez SE, Lam N, Garcia P, Williams MA. Associations of depression and depressive symptoms with preeclampsia: results from a Peruvian case-control study. BMC women's health. 2007;7:15.

2. Buist A. Managing depression in pregnancy. Australian family physician. 2000;29:663-7.

3. Limlomwongse $\mathrm{N}$, Liabsuetrakul T. Cohort study of depressive moods in Thai women during late pregnancy and 6-8 weeks of postpartum using the Edinburgh Postnatal Depression Scale (EPDS). Archives of women's mental health. 2006;9:131-8.

4. Gaynes BN, Gavin N, Meltzer-Brody S, Lohr KN, Swinson T, Gartlehner G, et al. Perinatal depression: prevalence, screening accuracy, and screening outcomes. Evid Rep Technol Assess (Summ). 2005;1-8.

5. Stuart S, Couser G, Schilder K, O'Hara MW, Gorman L. Postpartum anxiety and depression: onset and comorbidity in a community sample. The Journal of nervous and mental disease. 1998;186:420-4.

6. Benute GR, Nomura RM, Reis JS, Fraguas Junior R, Lucia MC, Zugaib M. Depression during pregnancy in women with a medical disorder: risk factors and perinatal outcomes. Clinics (Sao Paulo). 2010;65:112731.

7. Kersting A, Kroker K, Steinhard J, Hoernig-Franz I, Wesselmann U, Luedorff K, et al. Psychological impact on women after second and third trimester termination of pregnancy due to fetal anomalies versus women after preterm birth--a 14-month follow up study. Archives of women's mental health. 2009;12:193-201.

8. Le Strat Y, Dubertret C, Le Foll B. Prevalence and correlates of major depressive episode in pregnant and postpartum women in the United States. Journal of affective disorders. 2011;135:128-38.

9. Poel $\mathrm{YH}$, Swinkels $\mathrm{P}$, de Vries JI. Psychological treatment of women with psychological complaints after pre-eclampsia. Journal of psychosomatic obstetrics and gynaecology. 2009;30:65-72.

10. Rahman A, Iqbal Z, Bunn J, Lovel H, Harrington R. Impact of maternal depression on infant nutritional status and illness: a cohort study. Archives of general psychiatry. 2004;61:946-52. 
11. Weinberg MK, Tronick EZ. The impact of maternal psychiatric illness on infant development. The Journal of clinical psychiatry. 1998;59 Suppl 2:53-61.

12. Bonari L, Pinto N, Ahn E, Einarson A, Steiner M, Koren G. Perinatal risks of untreated depression during pregnancy. Canadian journal of psychiatry Revue canadienne de psychiatrie. 2004;49:726-35.

13. Cooper PJ, Campbell EA, Day A, Kennerley H, Bond A. Non-psychotic psychiatric disorder after childbirth. A prospective study of prevalence, incidence, course and nature. The British journal of psychiatry : the journal of mental science. 1988;152:799-806.

14. Kelly R, Zatzick D, Anders T. The detection and treatment of psychiatric disorders and substance use among pregnant women cared for in obstetrics. The American journal of psychiatry. 2001;158:213-9.

15. Blom EA, Jansen PW, Verhulst FC, Hofman A, Raat H, Jaddoe VW, et al. Perinatal complications increase the risk of postpartum depression. The Generation R Study. BJOG : an international journal of obstetrics and gynaecology. 2010;117:1390-8.

16. Engelhard IM, van Rij M, Boullart I, Ekhart TH, Spaanderman ME, van den Hout MA, et al. Posttraumatic stress disorder after pre-eclampsia: an exploratory study. General hospital psychiatry. 2002;24:260-4.

17. Brusse I, Duvekot J, Jongerling J, Steegers E, De Koning I. Impaired maternal cognitive functioning after pregnancies complicated by severe pre-eclampsia: a pilot case-control study. Acta obstetricia et gynecologica Scandinavica. 2008;87:408-12.

18. Hannaford P, Ferry S, Hirsch S. Cardiovascular sequelae of toxaemia of pregnancy. Heart. 1997;77:154-8.

19. Wells G, Shea B, O'Connell D, Peterson J, Welch V, Losos M, et al. The Newcastle-Ottawa Scale (NOS) for assessing the quality of nonrandomised studies in meta-analyses. http://www.ohri.ca/programs/clinical_epidemiology/oxford.asp: Ottawa Health Research Institute,

20. Rothman KJ, Greenland S, Lash TL, contributors w. Modern epidemiology, third edition. Philadelphia: Lippincott-Raven, 2008.

21. Hincz P, Borowski D, Krekora M, Podciechowski L, Horzelski W, Wilczynski J. Maternal obesity as a perinatal risk factor. Ginekologia polska. 2009;80:334-7.

22. Scott KM, McGee MA, Wells JE, Oakley Browne MA. Obesity and mental disorders in the adult general population. Journal of psychosomatic research. 2008;64:97-105.

23. Hasler G, Pine DS, Gamma A, Milos G, Ajdacic V, Eich D, et al. The associations between psychopathology and being overweight: a 20-year prospective study. Psychological medicine. 2004;34:1047-57.

24. Silva LM, Coolman M, Steegers EA, Jaddoe VW, Moll HA, Hofman A, et al. Low socioeconomic status is a risk factor for preeclampsia: the Generation R Study. Journal of hypertension. 2008;26:1200-8.

25. Goyal D, Gay C, Lee K. How much does low socioeconomic status increase the risk or prenatal and postpartum depressive symptoms in first-time mothers? Womens Health Issues. 2010;20:9.

26. Brewin CR, Andrews B, Valentine JD. Meta-analysis of risk factors for posttraumatic stress disorder in trauma-exposed adults. Journal of consulting and clinical psychology. 2000;68:748-66.

27. Lim L, Ng TP, Chua HC, Chiam PC, Won V, Lee T, et al. Generalised anxiety disorder in Singapore: prevalence, co-morbidity and risk factors in a multi-ethnic population. Social psychiatry and psychiatric epidemiology. 2005;40:972-9.

28. Wijma K, Soderquist J, Wijma B. Posttraumatic stress disorder after childbirth: a cross sectional study. Journal of anxiety disorders. 1997;11:587-97.

29. Qiu C, Williams MA, Calderon-Margalit R, Cripe SM, Sorensen TK. Preeclampsia risk in relation to maternal mood and anxiety disorders diagnosed before or during early pregnancy. American journal of hypertension. 2009;22:397-402.

30. Lewinsohn PM, Roberts RE, Seeley JR, Rohde P, Gotlib IH, Hops H. Adolescent psychopathology: II. Psychosocial risk factors for depression. Journal of abnormal psychology. 1994;103:302-15.

31. Duckitt K, Harrington D. Risk factors for pre-eclampsia at antenatal booking: systematic review of controlled studies. BMJ. 2005;330:565.

32. Ros H, Cnattingius S, Lipworth L. Comparison of risk factors for preeclampsia and gestational hypertension in a population-based cohort study. Am J Epidemiol. 1998;147:1062-70. 
33. Schneider S, Freerksen N, Maul H, Roehrig S, Fischer B, Hoeft B. Risk groups and maternal-neonatal complications of preeclampsia--current results from the national German Perinatal Quality Registry. Journal of perinatal medicine. 2011;39:257-65.

34. Rich-Edwards JW, Kleinman K, Abrams A, Harlow BL, McLaughlin TJ, Joffe H, et al. Sociodemographic predictors of antenatal and postpartum depressive symptoms among women in a medical group practice. Journal of epidemiology and community health. 2006;60:221-7.

35. Eskenazi B, Fenster L, Sidney S. A multivariate analysis of risk factors for preeclampsia. JAMA : the journal of the American Medical Association. 1991;266:237-41.

36. Righetti-Veltema M, Conne-Perreard E, Bousquet A, Manzano J. Risk factors and predictive signs of postpartum depression. Journal of affective disorders. 1998;49:167-80.

37. Susan A, Harris R, Sawyer A, Parfitt Y, Ford E. Posttraumatic stress disorder after childbirth: analysis of symptom presentation and sampling. Journal of affective disorders. 2009;119:200-4.

38. Caughey $A B$, Stotland NE, Washington AE, Escobar GJ. Maternal ethnicity, paternal ethnicity, and parental ethnic discordance: predictors of preeclampsia. Obstetrics and gynecology. 2005;106:156-61.

39. Horowitz JA, Murphy CA, Gregory KE, Wojcik J. A community-based screening initiative to identify mothers at risk for postpartum depression. Journal of obstetric, gynecologic, and neonatal nursing : JOGNN / NAACOG. 2011;40:52-61.

40. Faisal-Cury A, Rossi Menezes P. Prevalence of anxiety and depression during pregnancy in a private setting sample. Archives of women's mental health. 2007;10:25-32.

41. Baecke M, Spaanderman ME, van der Werf SP. Cognitive function after pre-eclampsia: an explorative study. Journal of psychosomatic obstetrics and gynaecology. 2009;30:58-64.

42. Gaugler-Senden IP, Duivenvoorden HJ, Filius A, De Groot CJ, Steegers EA, Passchier J. Maternal psychosocial outcome after early onset preeclampsia and preterm birth. The journal of maternal-fetal \& neonatal medicine : the official journal of the European Association of Perinatal Medicine, the Federation of Asia and Oceania Perinatal Societies, the International Society of Perinatal Obstet. 2011;

43. Stramrood CA, Wessel I, Doornbos B, Aarnoudse JG, van den Berg PP, Schultz WC, et al. Posttraumatic stress disorder following preeclampsia and PPROM: a prospective study with 15 months follow-up. Reprod Sci. 2011;18:645-53.

44. Boyd RC, Le HN, Somberg R. Review of screening instruments for postpartum depression. Archives of women's mental health. 2005;8:141-53.

45. Mitchell A, Coyne J. Screening for depression in clinical practice. An evidence-based guideline. Oxford: Oxford University Press, 2010.

46. Kurki T, Hiilesmaa V, Raitasalo R, Mattila H, Ylikorkala O. Depression and anxiety in early pregnancy and risk for preeclampsia. Obstetrics and gynecology. 2000;95:487-90.

47. Mori T, Tsuchiya KJ, Matsumoto K, Suzuki K, Mori N, Takei N. Psychosocial risk factors for postpartum depression and their relation to timing of onset: the Hamamatsu Birth Cohort (HBC) Study. Journal of affective disorders. 2011;135:341-6. 
CHAPTER 7

Summary and General discussion

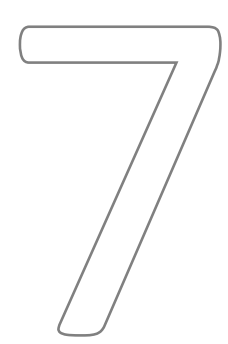


The studies presented in this thesis were centered around comparing the psychological and economic effects of obstetrical care-as-usual (CAU) with obstetrical care based on a risk-guided guideline (RGC) in women with a history of PE/HELLP in the Netherlands. The latter form of care was integrated with a newly developed prediction rule for estimation of recurrence risk of PE/HELLP. Although earlier studies on single predictors for recurrent PE/HELLP consistently reported their limited sensitivity, multi-parameter tools for the prediction of recurrent PE/HELLP have only recently become available (1). Clinical guideline 06 of the Dutch Society of Obstetrics and Gynecology (NVOG) only focuses on hypertensive disorders in pregnancy developing for the first time (2), providing recommendations for the use of antihypertensive drugs, corticosteroids, the prophylactic use of magnesium sulphate, and how to counsel the mother about the prognosis of her unborn child. However, to the best of our knowledge, there is no such guideline for prenatal care of women with a history of PE/HELLP, even though pregnant women with a history of PE/HELLP are at increased risk of recurrent PE/HELLP and other placental syndromes. This lack of a standardized management directive was the most important motive to set up the PreCare study. In addition to reporting on the PreCare study, we critically reviewed the literature about mental health problems among women with a history of PE/HELLP.

\section{Main findings}

In this thesis, the following topics are presented in consecutive order: study design of the PreCare study (Chapter 2), description of CAU (Chapter 3), cost-effectiveness analysis of RGC versus CAU (Chapter 4), patient satisfaction with RGC (Chapter 5), systematic review on anxiety and depression after PE/HELLP (Chapter 6).

Chapter 2 elaborates on the study protocol. The PreCare study uses a before-after design, in which pregnancy outcome and costs are compared between CAU and RGC. In $\mathrm{CAU}$, gynecologists did not receive any instructions and were requested to manage their patients in whatever manner they deemed appropriate. RGC consisted of two levels of care intensity, with Medium Care (MC) including 9 to 11 outpatient visits, and High Care $(\mathrm{HC})$ including 14 to 16 outpatient visits plus optional additional diagnostics. For a cost-effectiveness analysis with a time horizon of one year (start pregnancy-3 months postpartum), we calculated two incremental cost-effectiveness ratios (ICERs) expressing 1) the cost per Quality Adjusted Life Year, i.e. QALY (mother unit of analysis), performed from the societal perspective, and 2) the cost per prevented neonatal adverse outcome, i.e. NICU admission and/or death (child unit of analysis), performed from the hospital perspective. 
Chapter 3 describes hospital resource usage in terms of diagnostic tests, outpatient visits, admissions, deliveries and pregnancy-related costs in a historical cohort of pregnant women who had a previous preeclamptic pregnancy and who were treated according to CAU. The chapter also evaluates how this resource usage is related to pregnancy outcome. We focused our analysis on the outcome and costs from the start of pregnancy until delivery, as hospital care required by the newborn is excluded from obstetrical CAU provided by gynecologists/obstetricians. Maternal hospital costs per patient averaged $€ 8,047$, with main cost drivers being maternal admissions and maternal outpatient visits, which together comprised over $80 \%$ of the total hospital costs. Costs are particularly high in conjunction with preterm birth and/or recurrent PE/HELLP. The content of CAU for pregnant women with a history of PE/HELLP varied considerably, with the number of outpatient visits ranging from 5 to as many as 37 . Although multiple regression analyses indicated that some pregnancy outcomes impacted maternal hospital costs, most of the variation in maternal hospital costs remained unexplained. Although variables not measured in this study (e.g. patient preferences, doctor practice style, local hospital policy) may explain some of this variation, most of it is likely to remain unexplainable. The latter supports the view that clinical management of this particular patient population may be improved by e.g. developing an evidence-based guideline.

In Chapter 4, RGC was compared with CAU with respect to costs, effects and costeffectiveness. The study used a before-after design. Two incremental ICERs were calculated expressing 1) the cost per QALY (mother unit of analysis), from the societal perspective, and 2) the cost per prevented adverse neonatal outcome, i.e. NICU admission and/or death (child unit of analysis), from the hospital perspective. The base-case results of the cost per QALY analysis show that RGC was not cost-effective compared to CAU from a societal perspective due to both lower QALYs and higher costs in the RGC group. Bootstrap results showed that effectiveness was also consistently lower in the RGC group (99\% of replications) with societal costs being higher for RGC for almost $80 \%$ of the replications. From the hospital perspective, the cost per prevented adverse outcome analysis indicated RGC to be more cost-effective than CAU, mainly due to lower hospital costs. In most cases, sensitivity and subgroup analyses for both perspectives confirmed the results of the base-case analyses.

In Chapter 5, we explored whether patients in the RGC group were satisfied about the care received, about the factors contributing to patients' level of satisfaction and whether level of satisfaction influenced protocol adherence. Patient satisfaction scores were high for each subscale of the PSQ III. Differences between HC and MC patients were small and inconsistent. However, after correction for potential confounders, HC patients were more satisfied than MC patients about "time spent with the doctor". Patients' expectations/previous experiences, baseline psychological health status and 
being assigned to either $\mathrm{HC}$ or MC had a significant impact on satisfaction. Only satisfaction with "communication" appeared to be a predictor of adherence score.

Chapter 6 presented a systematic review evaluating whether having experienced PE/HELLP predisposed to anxiety and depression. A better insight into the development and severity of mental health problems in former PE/HELLP patients may help to develop more effective prevention and management strategies. A literature search was conducted in PubMed and Psyclnfo with no limit regarding date of publication, but limited to studies reported in English and containing an abstract. The search yielded 267 articles, with only six suitable for inclusion in this review. A critical appraisal checklist was used to facilitate data extraction and to evaluate the studies' appropriateness with respect to our research questions. Studies on post PE/HELLP depression $(n=6)$ showed generally positive associations between PE/HELLP and the prevalence of depression or severity of depressive symptoms, but the associations were statistically insignificant in half of the studies. Studies addressing anxiety $(n=2)$ showed increased severity scores after PE/HELLP, but all evaluated associations were statistically insignificant. Studies on posttraumatic stress showed in general a higher prevalence and higher severity scores of posttraumatic stress, but again, these findings were often statistically insignificant. Thus, even though the reported associations did not reach statistical significance, the direction of almost all these associations was positive. The latter provides some indirect support for the view that a history of PE/HELLP may predispose to the subsequent development of various forms of psychopathology.

\section{Reflection on the results}

The results of the PreCare study support the concept that -irrespective of pregnancy outcome- standardization of care for pregnant women with a history of early-onset PE/HELLP may reduce the huge variation in maternal hospital costs (Chapter 3). It may also be more cost-effective than CAU from a hospital perspective (Chapter 4). On the other hand, from a societal perspective, CAU is preferred over RGC, which is reflected in higher QALYs and less maternal health care costs outside the hospital in the CAU group. Especially women assigned to MC reported lower QALYs and higher health carerelated expenses outside the hospital. Interestingly, MC patients were also significantly less satisfied with "time spent with doctor" compared to HC patients (Chapter 5). Our systematic review on the available evidence about the severity and occurrence of anxiety and depression in patients who experienced a pregnancy complicated by PE/HELLP suggested that these women may be prone to more postpartum anxiety and depression, although the evidence for such a predisposition awaits confirmation in a sufficiently powered prospective study (Chapter 6). 
All women included in the PreCare study had a previous pregnancy complicated by early-onset PE/HELLP. Therefore, these women can be expected to be more anxious than their counterparts with a preceding uneventful first pregnancy. It is possible that these women systematically interpret ambiguous information in the informed consent document as more threatening than women with a history of uneventful pregnancies only (3). A restriction in the number of outpatient visits in the $\mathrm{MC}$ protocol (relative to $\mathrm{HC}$ and $\mathrm{CAU}$ ) may be experienced as threatening. Furthermore, MC women may have perceived a loss in optimal care provision in conjunction with the restriction in number of anticipated outpatient visits. This phenomenon is referred to as "loss aversion", where losses have greater subjective weight than equivalent gains (4). As a result, MC patients may report on average lower patient satisfaction scores with "time spent with doctor", higher costs outside the hospital and lower QALYs. These issues may explain the lower cost-effectiveness in RGC relative to CAU (societal perspective).

\section{Methodological considerations}

In interpreting the results of this thesis, it is relevant to consider the methodology used to obtain the data. Here, we want to elaborate on the validity of the prediction model, the use of a before-after design, the size of the group for the prospective CAU study, the absence of a comparison group in the study on satisfaction with RGC and protocol adherence, topics which have not been addressed in detail in previous chapters.

\section{Prediction model}

For the purpose of this study, we developed a prediction model, which would allow us to differentiate between former patients who are and are not at high risk to develop recurrent PE in their next pregnancy (5). This approach would allow us to offer RGC to former patients and enable us to determine, whether this more or less customized care would be superior over CAU regarding cost-effectiveness, patient satisfaction and pregnancy outcome. Unfortunately, this prediction model discriminated poorly between low-risk and high-risk former patients and therefore, in its present form, was unsuitable for introduction in clinical practice to serve as an adequate basis for RGC.

\section{Before-after design}

Although the preferred design for evaluating the implementation of a new care strategy would be a randomized controlled trial (RCT), we chose a before-after design for several reasons. In the case of PreCare, an RCT would mean that CAU or RGC would be offered at random either at patient level or at hospital level. A study randomized on a 
patient level would have been vulnerable to contamination, implying that gynecologists would be able to apply the same knowledge about the risk of recurrence of patients assigned to the CAU group as of patients assigned to the RGC group, and possibly use this knowledge as a guide for the intensity of antenatal care, thereby diminishing any possible effects between CAU and RGC (6). A study randomized on hospital level would have been sensitive to local protocols on care in the hospitals assigned to the CAU group, or the adherence to protocols offered to hospitals assigned to the RGC group. Therefore, a before-after study design was chosen in which at least all hospitals that participated in the before part, would also participate in the part after implementation of the prediction rule. This approach circumvents the problem of bias introduced by large differences between hospitals.

\section{Small group for prospective CAU}

In the CAU group, patients were followed both prospectively and retrospectively. We chose to identify most CAU patients retrospectively using hospital information systems and medical records in order to save time to complete the study. These patients had been diagnosed with PE/HELLP between 1996 and 2012 and thus, had received CAU. The women in this retrospective CAU group and those in the RGC group were matched with one another for age, gestational age of birth in the previous pregnancy and center. As a consequence, the number of CAU patients followed prospectively was relatively small. Only prospectively followed patients were able to fill out questionnaires regarding costs outside the hospital, health-related quality of life, anxiety, depression and posttraumatic stress. The use of smaller groups may come with greater uncertainty as regards cost-effectiveness results; this was however appropriately handled using bootstrap analysis.

\section{Comparison of satisfaction between RGC and CAU was not possible}

In our satisfaction study, we could not compare satisfaction between CAU and RGC as we did not measure satisfaction in the CAU group. The reason for this is that the larger part of the CAU data were obtained from a historical cohort of patients and these patients did not receive patient satisfaction questionnaires (see above). By the time the satisfaction questionnaire was in its final form, i.e. the questions which were specifically formulated for evaluation of PreCare, the prospective CAU patients were already included. Unfortunately, we were unable to explore whether RGC patients were more satisfied than CAU patients. 


\section{Protocol adherence}

Despite considerable efforts to promote maximum compliance of the participating gynecologists with our protocols, adherence to the MC and HC protocols was poor. Nowadays, most studies focus on outcome rather than on the process involved in implementing an intervention, treatment option or protocol (7). Conventional costeffectiveness analyses explore whether a new treatment is cost-effective. However, only few studies disentangle the factors that ensure successful outcomes, characterize the failure to achieve success, or attempt to document the steps in achieving successful implementation of an intervention, new treatment or protocol (8-11). Process evaluations within studies explore the implementation, receipt and setting of a new treatment option and facilitate the interpretation of the outcome results. Such process evaluation was not intended in the PreCare study. However, it may provide an important policy message.

Process evaluations examine the views of the participants (doctors, patients) and study how the intervention is implemented. Several methods can be used to collect process data, for example focus groups, interviews or questionnaire surveys. Particularly in case of an unexpected or disappointed outcome, researchers and funders would like to understand why the expected outcome was not achieved. Thus, integrating process and outcome data can help to interpret unexpected study results.

\section{Recommendations for future research and clinical practice}

Our prediction model performed poorly in women with a history of early-onset PE/HELLP (12). A challenge for future research would be to develop a new prediction rule with better discriminatory performance. If such a test becomes available, a logical next step would be to explore whether a newly developed RGC is more cost-effective than the current CAU. If initial positive study results are confirmed by more than one study group in large numbers, recommendations can be included in guidelines and provide a new starting point for further research. It is well known that newly developed prediction models require external validation because of design deficiencies or overfitting (13). Ideally, the PreCare study would have been designed with an integrated process evaluation, taking into account the views of doctors and patients. Additionally, our study provides support for the concept that being informed ahead about possible barriers and facilitators for implementation is likely to improve protocol adherence $(14,15)$.

RGC differed from CAU by higher expenses outside the hospital, a lower healthrelated quality of life and probably a lower patient satisfaction score (especially in the subgroup of MC patients). The latter was probably related to a lower allowance for 
outpatient visits in $\mathrm{MC}$ than in $\mathrm{HC}$ and this may have urged these patients to consult alternative health care providers outside the hospital. Therefore, future protocols should also take into account (health) care outside the hospital. For example, it would be interesting to offer preventive mental health measures to women with a history of early-onset PE/HELLP, thus helping them to cope better with the stress associated with a new pregnancy. These patients with a higher risk for anxiety and depression may benefit from tailored information via internet or same-day-call-back service by the obstetrician or a specialized nurse practitioner. This strategy will not only reduce this apparent extra need for regular reassurance. It will also prevent them from consulting other health care providers (outside the hospital). Nowadays, gynecologists give priority to the patient's clinical condition. However, the systematic review conducted alongside the PreCare study shows that attention for anxiety and depression should not be ignored. Only a small percentage of these women will develop an early-onset recurrence. However, all former patients had been exposed to stressful situations in their previous pregnancy. Prevention and timely recognition may reduce treatment costs (outside the hospital), improve health-related quality of life and patient satisfaction.

\section{References}

1. Sep S, Smits L, Prins M, Peeters L. Prediction tests for recurrent hypertensive disease in pregnancy, a systematic review. Hypertension in pregnancy : official journal of the International Society for the Study of Hypertension in Pregnancy. 2010 Jan;29(2):206-30.

2. NVOG. Richtlijn 6 - Hypertensieve aandoeningen in de zwangerschap. 2005.

3. Taghavi MR, Moradi AR, Neshat-Doost HT, Yule W, Dalgleish T. Interpretation of ambiguous emotional information in clinically anxious children and adolescents. Cognition \& Emotion. 2000;14(6):809-22.

4. Kahneman D, Tversky A. Prospect theory: An analysis of decision under risk. Econometrica. 1979;47:26391.

5. van Kuijk SM, Nijdam ME, Janssen KJ, Sep SJ, Peeters LL, Delahaije DH, et al. A model for preconceptional prediction of recurrent early-onset preeclampsia: derivation and internal validation. Reprod Sci. 2011 Nov;18(11):1154-9.

6. Grimshaw J, Campbell M, Eccles M, Steen N. Experimental and quasi-experimental designs for evaluating guideline implementation strategies. Family practice. 2000 Feb;17 Suppl 1:S11-6.

7. Oakley A, Strange V, Bonell C, Allen E, Stephenson J, Team RS. Process evaluation in randomised controlled trials of complex interventions. BMJ. 2006 Feb 18;332(7538):413-6.

8. Volpe SL, Hall WJ, Steckler A, Schneider M, Thompson D, Mobley C, et al. Process evaluation results from the HEALTHY nutrition intervention to modify the total school food environment. Health education research. 2013 Dec;28(6):970-8.

9. Branscum P, Sharma M, Wang LL, Wilson B, Rojas-Guyler L. A process evaluation of a social cognitive theory-based childhood obesity prevention intervention: the Comics for Health program. Health promotion practice. 2013 Mar;14(2):189-98.

10. McCreary LL, Kaponda CP, Kafulafula UK, Ngalande RC, Kumbani LC, Jere DL, et al. Process evaluation of HIV prevention peer groups in Malawi: a look inside the black box. Health education research. 2010 Dec;25(6):965-78. 
11. Rosecrans AM, Gittelsohn J, Ho LS, Harris SB, Naqshbandi M, Sharma S. Process evaluation of a multiinstitutional community-based program for diabetes prevention among First Nations. Health education research. 2008 Apr;23(2):272-86.

12. van Kuijk SM, Delahaije DH, Dirksen CD, Scheepers HC, Spaanderman ME, Ganzevoort W, et al. External validation of a model for periconceptional prediction of recurrent early-onset preeclampsia. Hypertension in pregnancy : official journal of the International Society for the Study of Hypertension in Pregnancy. 2014 Jan 6.

13. Altman DG, Vergouwe $Y$, Royston P, Moons KG. Prognosis and prognostic research: validating a prognostic model. BMJ. 2009;338:b605.

14. Grol R. Improving the quality of medical care: building bridges among professional pride, payer profit, and patient satisfaction. JAMA : the journal of the American Medical Association. 2001 Nov 28;286(20):2578-85.

15. Kimman ML, Bloebaum MM, Dirksen CD, Houben RM, Lambin P, Boersma LJ. Patient satisfaction with nurse-led telephone follow-up after curative treatment for breast cancer. BMC cancer. 2010;10:174. 

Nederlandstalige samenvatting 
In hoofdstuk 1 van dit proefschrift worden de zwangerschapsaandoeningen preeclampsie en het HELLP syndroom, het vóórkomen, het herhalingsrisico in een volgende zwangerschap en de mogelijkheid om een herhaling te voorspellen kort geïntroduceerd. Het hoofdstuk wordt afgesloten met de specifieke doelstellingen en inhoud van het proefschrift.

Bij preëclampsie is er naast hoge bloeddruk in de zwangerschap ook sprake van eiwitverlies in de urine. Het HELLP-syndroom staat voor "Hemolysis, Elevated Liver enzymes en Low Platelets". Dit betekent dat er sprake is van een verhoogde afbraak van rode bloedcellen en een gestoorde leverfunctie. Daarnaast is er een tekort aan bloedplaatjes, waardoor de bloedstolling ontregeld raakt. Sommige vrouwen hebben lange tijd weinig of geen klachten, anderen worden in korte tijd ernstig ziek. Preeclampsie en het HELLP-syndroom kunnen ook ernstige gevolgen hebben voor het kind, zoals groeivertraging en vroeggeboorte. Hoewel bekend is dat preëclampsie en het HELLP-syndroom ontstaan door onvoldoende werking van de placenta (moederkoek), is de exacte oorzaak nog niet duidelijk. Vrouwen die eenmaal preëclampsie of het HELLP-syndroom hebben doorgemaakt, hebben een verhoogde kans op herhaling in de volgende zwangerschap. Er zijn in Nederland geen klinische praktijk-richtlijnen voor de begeleiding van zwangere vrouwen met een dergelijke complicatie in de voorgeschiedenis. Veelal krijgen deze vrouwen zeer intensieve en kostbare zorg, terwijl dat in de meeste gevallen misschien niet nodig is. Vrouwen met een laag herhalingsrisico zouden misschien reguliere zorg kunnen krijgen, terwijl voor vrouwen met een hoog herhalingsrisico een dergelijke intensieve zorg wel te rechtvaardigen is. Om onderscheid te maken tussen vrouwen met een laag herhalingsrisico en vrouwen met een hoog herhalingsrisico, werd een predictiemodel ontwikkeld. In de PreCare studie werden de effecten van dit predictiemodel verder onderzocht op het gebied van uitkomsten bij moeder en kind, patiënttevredenheid en maatschappelijke en zorgkosten (recidiefkans-geleide zorg, of RGC). Op basis van dit predictiemodel werden vrouwen ingedeeld in Medium Care (MC) of High Care ( $\mathrm{HC}$ ), afhankelijk van het voorspelde individuele risico om een recidief te ontwikkelen. Naderhand werd recidiefkans-geleide zorg vergeleken met de gebruikelijke zorg (CAU) met betrekking tot zwangerschapsgerelateerde zorgkosten en maatschappelijke kosten (zoals ziekteverzuim, informele zorg) en gezondheidsuitkomsten bij moeder en kind en werd de patiënttevredenheid met recidiefkans-geleide zorg onderzocht.

Hoofdstuk 2 geeft een gedetailleerde beschrijving van de opzet en uitvoering van de studie. De PreCare-studie hanteert een zogenaamd before-after design, waarin zwangerschapsuitkomsten en kosten worden vergeleken tussen de gebruikelijke zorg en recidiefkans-geleide zorg. In het kader van de gebruikelijke zorg wordt gynaecologen gevraagd om de patiënten te behandelen zoals gebruikelijk. Recidiefkans-geleide zorg 
bestaat uit twee zorgvarianten passend bij het risico op herhaling van preëclampsie of HELLP, namelijk MC bestaande uit 9-11 polibezoeken, en HC bestaande uit 14-16 polibezoeken plus eventuele additionele diagnostiek. Voor de economische evaluatie met een tijdshorizon van een jaar (begin van de zwangerschap tot 3 maanden na de bevalling) worden twee incrementele kosteneffectiviteitsratio's berekend, waarin de kosten per QALY (voor kwaliteit gecorrigeerde levensjaren) en de kosten per voorkomen nadelige neonatale uitkomst (NICU-opname en/of dood) worden vergeleken.

In hoofdstuk 3 wordt het zorggebruik in termen van diagnostische testen, polibezoeken, opnames, bevallingen en daaraan gerelateerde kosten beschreven binnen een cohort zwangere vrouwen. Deze vrouwen hadden een vorige zwangerschap gehad die gecompliceerd werd door preëclampsie of HELLP-syndroom en werden in het verleden behandeld volgens de gebruikelijke zorg. Dit hoofdstuk laat ook zien hoe dit zorggebruik samenhangt met zwangerschapsuitkomsten. De analyse was gericht op de uitkomsten en kosten van de moeder vanaf het begin van de zwangerschap tot en met de bevalling, omdat de zorg voor het kind geen deel uit maakt van de zorg door de gynaecoloog. De gemiddelde ziekenhuiskosten per patiënt bedroegen €8.047, met als belangrijkste kostencomponent opnames en polikliniekbezoeken (samen verantwoordelijk voor meer dan $80 \%$ van de totale kosten). Kosten waren vooral hoog in geval van vroeggeboorte en/of een recidief preëclampsie of HELLP-syndroom. De praktijkvariatie binnen de gebruikelijke zorg bleek aanzienlijk. Het aantal polibezoeken varieerde van 5 tot 37. Hoewel meervoudige regressieanalyses aantoonden dat er een verband is tussen sommige zwangerschapsuitkomsten en ziekenhuiskosten, blijft het merendeel van de spreiding onverklaard. Een deel van de spreiding wordt mogelijk verklaard door variabelen die niet gemeten zijn in de PreCare studie, zoals patiëntvoorkeuren, voorkeuren en routine van de dokter of lokaal ziekenhuisbeleid. Dit kan er op wijzen dat de zorg voor deze patiëntengroep kan worden verbeterd door het ontwikkelen van een klinische praktijk-richtlijn.

Hoofdstuk 4 presenteert de economische evaluatie waarin recidiefkans-geleide zorg vergeleken werd met de gebruikelijke zorg op het gebied van kosten, effecten en kosteneffectiviteit. Uikomsten werden uitgedrukt in kosten per QALY (vanuit een maatschappelijk perspectief) en kosten per voorkomen nadelige neonatale uitkomst (vanuit ziekenhuis perspectief). Vanuit het maatschappelijk perspectief lieten resultaten zien dat RGC niet kosteneffectief was in vergelijking met de gebruikelijke zorg door hogere kosten en lagere QALY's in de recidiefkans-geleide zorg-groep. Bootstrapanalyses toonden aan dat de effectiviteit consistent lager was in de recidiefkans-geleide zorggroep (99\% van de replicaties) met hogere maatschappelijke kosten voor recidiefkansgeleide zorg in ongeveer $80 \%$ van de replicaties. Vanuit het ziekenhuis perspectief 
bleek dat recidiefkans-geleide zorg juist kosteneffectief was in vergelijking met de gebruikelijke zorg, voornamelijk door lagere ziekenhuiskosten. Sensitiviteits- en subgroepanalyses lieten zien dat de resultaten robuust waren.

Hoofdstuk 5 beschrijft de mate van tevredenheid van patiënten in de recidiefkansgeleide zorg-groep, welke factoren hebben bijgedragen aan tevredenheid en of tevredenheid van invloed was op de naleving van de $\mathrm{HC}$ en $\mathrm{MC}$ protocollen in de PreCare studie. Tevredenheidsscores werden verzameld bij inclusie en 10 dagen na de bevalling met de Nederlandse versie van Ware's Patiënt Tevredenheidsvragenlijst, de PSQ III. Scores waren hoog voor alle subschalen van de PSQ III, wat op een hoge tevredenheid duidt. Er werden geen betekenisvolle verschillen gevonden tussen HC- en MCpatiënten. Echter, na correctie voor mogelijke verstorende factoren, bleken HCpatiënten significant meer tevreden over "tijd besteed met de dokter" dan MCpatiënten. De verwachtingen, eerdere ervaringen, psychische gezondheidstoestand bij inclusie en ingedeeld worden in $\mathrm{HC}$ of $\mathrm{MC}$ hadden een significante invloed op tevredenheid. Alleen "tevredenheid met communicatie" bleek een voorspeller te zijn voor de naleving van de protocollen.

Hoofdstuk 6 biedt een literatuuroverzicht van de relatie tussen preëclampsie of HELLPsyndroom en posttraumatische stress, angst- en depressieve klachten. Een systematisch literatuuronderzoek werd uitgevoerd in PubMed en Psyclnfo. Uit 267 resultaten werden uiteindelijk slechts 6 studies geschikt geacht om te includeren in de review. Een checklist werd gebruikt om de data-extractie te vergemakkelijken en de geschiktheid van de artikelen in het licht van onze onderzoeksvragen te evalueren. Studies over depressie $(n=6)$ lieten positieve relaties zien tussen preëclampsie en HELLP syndroom en het vóórkomen van depressie of de ernst van de depressieve symptomen, maar in de helft van de studies waren de associaties niet statistisch significant. Studies over angst $(n=2)$ toonden hogere scores met betrekking tot de ernst, maar alle associaties waren statistisch niet significant. Studies over posttraumatische stress beschreven in het algemeen een hoger vóórkomen en ernstigere symptomen, maar weer waren deze resultaten vaak niet statistisch significant. Concluderend, hoewel de gerapporteerde relaties niet steeds statistisch significant waren, waren de meeste wel positief. Dit ondersteunt het beeld dat een doorgemaakte preëclampsie of HELLP-syndroom mogelijk effect heeft op het ontstaan van diverse vormen van psychopathologie.

Hoofdstuk 7 levert ten slotte een samenvatting en een algemene discussie over het onderzoek dat in dit proefschrift is beschreven.

Een belangrijk aandachtsgebied voor toekomstig onderzoek is het ontwikkelen van een verbeterd predictiemodel om vervolgens te onderzoeken of een aangepaste 
vorm van recidiefkans-geleide zorg kosteneffectief is ten opzichte van de gebruikelijke zorg. Aandacht voor mogelijke psychopathologie mag hierbij zeker niet ontbreken. Vanwege hoge kosten buiten het ziekenhuis in de huidige vorm van recidiefkansgeleide zorg, zouden toekomstige protocollen ook betrekking moeten hebben op de zorg buiten het ziekenhuis.

Idealiter zouden procesevaluaties voortaan moeten worden uitgevoerd binnen studies, om na te gaan wat de ervaringen zijn van alle betrokkenen (zowel artsen als patiënten) en wat de succesfactoren en knelpunten zijn. Dit zou aan de nieuwe vorm van recidiefkans-geleide zorg gekoppeld kunnen worden.

Voor de clinicus is het belangrijk om alert te zijn op de eventuele ontwikkeling van psychopathologie, omdat preventie en tijdige herkenning kosten kunnen besparen en kwaliteit van leven en patiënttevredenheid kunnen verhogen. 
Valorization 


\section{Valorization of research}

Over the last decade there is an ongoing debate about the societal impact and utilization of academic research. This is also called 'valorization of knowledge' and can be defined as "the process of value-creation out of knowledge, by making this knowledge suitable and available for economic or societal utilization and to translate this into high-potential products, services, processes and industrial activity" (adapted definition based on the National Valorisation Committee 2011:8). In the Netherlands, the standard of scientific research is high. However, this scientific knowledge is underutilized in practice. For example, only a limited number of scientific discoveries find their way to the market.

Apart from commercializing the new intervention through a spin-off company, patent, or license, there are many ways to make impact, for example through the transfer of knowledge to health care organizations, by making knowledge and expertise available for other organizations and by communicating knowledge to the general public.

A better utilization of knowledge (based on study results) is a valuable resource for the development of the economy and innovative capacity.

There is a growing focus on utilization of knowledge and scientific results, reflected by the policies within academia and knowledge institutes and important criteria against which publicly funded projects are judged.

Currently, our society faces big challenges in health care. The population is ageing and will increasingly face dangerous diseases like cancer and serious chronic diseases like diabetes. As a result, both a further improvement of our health care system and a severe reduction of costs are needed. To accomplish this, major innovations in tools for treatment and monitoring are required.

\section{The PreCare study}

Worldwide, preeclampsia (PE) and HELLP syndrome are one of the main causes of maternal and fetal mortality and morbidity $(1,2)$. Currently, the Dutch trends in maternal morbidity due to PE are a reason for great concern (3). It is thought that a high degree of maternal morbidity and mortality due to PE and HELLP syndrome result from suboptimal or insufficient treatment (4).

Pregnant women who had PE/HELLP in their previous pregnancy are at increased risk of developing a recurrence. At this moment, there is no clinical practice guideline for prenatal care for these women. This lack of a standardized guideline was the most important motive to set up the PreCare study. 
In the PreCare study, we developed a prediction model to identify women at really low risk of recurrence. Based on this model, women received either protocolized Medium Care or High Care, depending on their predicted risk. Medium Care and High Care together were called recurrence risk guided care (RGC). We compared RGC with care-asusual (CAU) for societal costs (health care and outside health care), quality of life, patient satisfaction and adverse maternal and neonatal outcomes. In addition to reporting on the PreCare study, we reviewed the literature about mental health problems among women with a history of PE/HELLP.

\section{Relevance}

The results of our study were somewhat disappointing, as CAU was preferred over RGC from the societal perspective in the cost-effectiveness analysis. For society, it is therefore not worth to introduce RGC into clinical practice yet. Nevertheless, for several reasons, our study is of scientific, economic and societal relevance.

Firstly, it is of scientific relevance since it leads to increased knowledge on the clinical, psychological and economic aspects of pregnancy care for women with a previous preeclamptic pregnancy. Furthermore, this study shows the gaps in current knowledge so that research activities can be prioritized. Evidence from the systematic review suggested that a history of PE/HELLP predisposes to subsequent development of various forms of psychopathology. However, further prospective studies are needed to confirm this view.

Despite considerable efforts to promote maximum compliance of the participating gynecologists with our protocols, adherence to the MC and HC protocols was poor. Unfortunately, the reasons were unclear since a process evaluation was not planned in the PreCare study. Therefore, we can recommend that process evaluations, in which the views of the participants are examined, should always be incorporated in future studies on health care quality improvements.

Additionally, this study is of economic relevance as it showed that there is a considerable (unnecessary and unwarranted) practice variation in CAU. Current care for pregnant women with a history of PE/HELLP is probably not efficient. Introducing standardized care (through clinical practice guidelines or protocols) is one strategy to decrease practice variation by providing the best available research evidence in the form of optimal care recommendations. Introducing guidelines can lead to significant cost reductions and unnecessary medical treatments. In general, about 20-25 percent of the care provided is not needed or potentially harmful (5). Our study demonstrated that practice variation in CAU could not be explained by women's characteristics or 
pregnancy outcomes. This indicates that there is room for improvement. Further research can make clear how costs can be reduced.

Finally, this study is of societal relevance since guideline implementation may lead to significant reductions in complications and medical errors. Quality of care received by pregnant women whose previous pregnancy was complicated by PE/HELLP can further be improved since this study has shown that attention should be paid to posttraumatic stress, anxiety and depression.

\section{Schedule and implementation}

Based on the results of this thesis, it is too early to develop clinical practice guidelines and implement them into clinical practice yet.

Because of disappointing study results and poor guideline adherence, it is necessary to perform a process evaluation concerning the PreCare study. Process evaluations within cost-effectiveness analyses explore the implementation, receipt and setting of a new intervention and help in the interpretation of the outcome results (6). They examine the views of participants, study how the intervention is implemented and investigate contextual factors that affect the intervention. More importantly, they can provide valuable insight into why an intervention fails or has unexpected consequences (7). Several methods can be used to collect process data, including questionnaire surveys, focus groups, interviews, researcher observations, and structured field notes. These will provide insights into possible barriers and facilitators for and satisfaction with the guidelines. Our results suggested that the lower cost-effectiveness in RGC relative to CAU (societal perspective) may be explained by a restricted number in outpatient visits in the MC protocol, reflected in lower patient satisfaction scores and higher costs outside the hospital among the MC group. Patients' experiences with these elements should therefore be measured.

A logical next step would be to develop a prediction model performing adequately in differentiating women according to their recurrence risk. The model we developed may be used as starting point that needs to be developed further to increase predictive performance. To do so, it might be updated with promising predictor variables that independently contribute to the model's discriminative ability and calibration measures, such as maternal thrombophilia and high-density lipoprotein (HDL) cholesterol $(8,9)$. Nevertheless, we must realize that it will be difficult at present to develop a robust prediction model because of the heterogeneous clinical presentation of PE and HELLP and their incompletely unraveled pathophysiology. Furthermore, prediction models tend to perform better on data on which the model was constructed (derivation set) than the same model on new data (validation set). 
Subsequently, based on the results of previous steps, a new guideline can be developed. Attention to possible development of psychopathology should be incorporated in the new guideline. For various reasons, however, guidelines have proven difficult to implement. The new guideline preferably is pilot tested before implementation and an inventory of the barriers and facilitators for adherence to the guideline is made. Barriers can arise at different stages in the health care system at the level of the individual gynecologist (i.e. lack of knowledge or skills, resistance against working with guidelines), of the team (i.e. lack of knowledge or skills, insufficient collaboration), of the patient (i.e. unwillingness, difficult guideline adherence for some patient groups), of the organization (i.e. lack of registration/information systems, time restriction for adequate care), and of the wider environment (i.e. lack of financial incentives, misuse of guideline in medical disciplinary law) and of the guideline itself (i.e. doubting evidence of the guideline, poor layout, unclear decision tree, unclear content).

The implementation strategy will be based on the barriers and facilitators from the previous step. Ideally, guidelines are developed to be disseminated and implemented in such a way that gynecologists and patients become aware of them and use them. Guidelines do not implement themselves; they need to be implemented actively. Well designed strategies are needed to optimize their success.

The protocols should be disseminated via various channels, both written and personal. The Dutch consortium for women's health and reproductive studies could facilitate publication of both the development process and the availability of the protocols. The protocols should be published in scientific journals and on the internet. Clinical leaders can be contacted to promote the protocols, because highly regarded individuals influence the practice of their peers. They can deal with misconceptions about guidelines and help clinicians observe the outcome of a particular innovation. Reminder systems should be incorporated in gynecologists' daily work, such as computerized prompting and mailed reminder systems. These reminders can be acted on immediately. Publicizing adherence to guidelines may improve public image, sending messages of commitment to excellence and quality. Feedback on compliance with the protocols can promote good will and potential support.

Strategies described above are probably more effective when used in combination. It is however important to notice that more research into the details of different implementation activities is needed to understand the critical determinants of change in clinical practice.

In summary, a process evaluation concerning the PreCare study should be performed. Next, a new prediction model should be developed with a better predictive performance. Based on the results of previous steps, a new guideline can be developed, incorporating attention to possible development of psychopathology. Finally, this guideline can be disseminated and implemented. 


\section{References}

1. Chhabra S, Kakani A. Maternal mortality due to eclamptic and non-eclamptic hypertensive disorders: a challenge. Journal of obstetrics and gynaecology : the journal of the Institute of Obstetrics and Gynaecology. 2007 Jan;27(1):25-9.

2. Moodley J. Maternal deaths associated with hypertensive disorders of pregnancy: a population-based study. Hypertension in pregnancy : official journal of the International Society for the Study of Hypertension in Pregnancy. 2004;23(3):247-56.

3. Schutte JM. Safe pregnancy in the Netherlands. NTOG. 2010;123:27-8.

4. Schutte JM, Schuitemaker NW, van Roosmalen J, Steegers EA, Dutch Maternal Mortality C. Substandard care in maternal mortality due to hypertensive disease in pregnancy in the Netherlands. BJOG : an international journal of obstetrics and gynaecology. 2008 May;115(6):732-6.

5. Richard G, Jeremy G. From best evidence to best practice: effective implementation of change in patients' care. Lancet. 2003;362:1225-30.

6. Oakley A, Strange V, Bonell C, Allen E, Stephenson J, Team RS. Process evaluation in randomised controlled trials of complex interventions. BMJ. 2006 Feb 18;332(7538):413-6.

7. Craig P, Dieppe P, Macintyre S, Michie S, Nazareth I, Petticrew M, et al. Developing and evaluating complex interventions: the new Medical Research Council guidance. BMJ. 2008;337:a1655.

8. Facchinetti F, Marozio L, Frusca T, Grandone E, Venturini P, Tiscia GL, et al. Maternal thrombophilia and the risk of recurrence of preeclampsia. American journal of obstetrics and gynecology. 2009 Jan;200(1):46 e1-5.

9. Sep SJ, Smits $\sqcup$, Prins MH, Spaanderman ME, Peeters LL. Simple prepregnant prediction rule for recurrent early-onset hypertensive disease in pregnancy. Reprod Sci. 2009 Jan;16(1):80-7. 



\section{Dankwoord}


In mijn studietijd had ik niet gedacht dat het zover zou komen, maar toch ligt hier na ruim 5 jaar mijn proefschrift! Het managen van deze grote multicenter studie vond ik een geweldige uitdaging. Promoveren doe je niet alleen, en daarom wil ik graag iedereen bedanken die direct of indirect aan mijn proefschrift heeft bijgedragen. Een aantal mensen wil ik graag in het bijzonder noemen.

Allereerst bedank ik natuurlijk mijn promoter prof. dr. C.D. Dirksen en copromotores dr. L.J.M. Smits en dr. L.L.H. Peeters. Jullie hebben in de totstandkoming van dit proefschrift een onmisbare rol gespeeld en hadden allemaal een positieve instelling toen de inclusie in het begin en de data-invoer op het eind niet helemaal liep zoals verwacht.

Beste Carmen, mijn dank gaat misschien wel het meest uit naar jou. Dank je voor het feit dat je me deze mogelijkheid hebt geboden. In de afgelopen jaren heb je het steeds drukker gekregen, toch kon ik altijd bij je terecht wanneer ik vragen had. Als jij er niet was geweest, dan had ik nu waarschijnlijk nog zitten zwoegen op de laatste loodjes.

Beste Luc, ik heb veel van je geleerd, zowel tijdens het schrijven van het review als van de vele besprekingen en je beoordeling van mijn stukken. Bedankt voor je verfrissende blik door jouw meer epidemiologische invalshoek en je altijd snelle en opbouwende reacties.

Beste Louis, jou wil ik eveneens hartelijk danken voor je vakbekwame begeleiding. Ik heb de manier waarop jouw klinische inbreng een invloed heeft gehad op de kwaliteit van mijn artikelen zeer gewaardeerd.

Beste Sander, jij bent mijn medepromovendus. Ik heb bijzonder veel plezier beleefd aan onze samenwerking. We hebben samen heel wat werk verzet en heel wat zorgen, files en syntaxen gedeeld. Ik heb je oprechte interesse in zowel privé als wetenschappelijke zaken altijd erg gewaardeerd. Nogmaals bedankt voor de prettige samenwerking en veel succes met je verdere carrière bij de GGD.

Mijn dank gaat ook uit naar dr. S.J.S. Sep, die aan de wieg stond van het PreCare project. Beste Simone, jij hebt het mede mogelijk gemaakt dat dit project er kwam door mee te denken over en mee te schrijven aan de subsidieaanvraag. Bedankt daarvoor.

De leden van mijn beoordelingscommissie prof. dr. S.M.A.A. Evers, prof. dr. J.G. Nijhuis, prof. dr. M.H. Prins, prof. dr. I.M. Engelhard en dr. A. Kwee wil ik hartelijk danken voor de bereidheid om mijn proefschrift kritisch te lezen en op wetenschappelijke waarde te beoordelen.

Graag wil ik alle gynaecologen en arts-assistenten bedanken uit de participerende ziekenhuizen: Academisch Ziekenhuis Maastricht, St. Radboud Ziekenhuis Nijmegen, Isala Klinieken Zwolle, Erasmus Medisch Centrum Rotterdam, Academisch Medisch Centrum Amsterdam, Onze Lieve Vrouwe Gasthuis Amsterdam, Universitair Medisch 
Centrum Utrecht, Maxima Medisch Centrum Veldhoven, Universitair Medisch Centrum Groningen, Amphia Ziekenhuis Breda, Atrium Medisch Centrum Heerlen en Kennemer Gasthuis Haarlem. Hartelijk dank voor jullie medewerking. Jullie hebben jarenlang meegewerkt aan de (soms moeizame) inclusie voor de studie en de bijbehorende follow-up.

Mijn dank gaat verder uit naar alle researchverloskundigen en -verpleegkundigen van de participerende ziekenhuizen voor hun grandioze en onuitputtelijke inzet. Corine Verhoeven, Jannet Bakker, Gerard Zijderveld, Joke van Rhee, Titia Winter, Diana Lutjes, José Keurentjes, Lida Ulkeman, David Borman, Maartje de Reus, Jolanda WillemsRobberts, Nathalie Teeuwen-Dedroog, Sabine Logtenberg, Birgit van der Goes, Joyce Cantineau: hartelijk dank voor het includeren van patiënten en het invoeren van alle data.

Zonder het Verloskundig Consortium was deze multicenter studie niet van de grond gekomen. Ik voel me ontzettend vereerd dat ik deel heb uit mogen maken van dit krachtige samenwerkingsverband van Nederlandse bodem. Ik wil de artsonderzoekers graag bedanken voor de goede samenwerking, de gezelligheid en de gedachtewisselingen tijdens onze driemaandelijkse bijeenkomsten in Utrecht.

De leden van de PreCare projectgroep (dr. L.C.J. Scheepers, dr. M.A. Oudijk, dr. J.J. Duvekot, dr. M.G. van Pampus, dr. W. Ganzevoort, prof. dr. M.E. Spaanderman, prof. dr. H.W. Bruinse, dr. L.D. de Wit-Zuurendonk, prof. dr. J.A.M. van der Post, dr. J. van Eyck, dr. M.A.B.H.M. van der Hoeven) wil ik graag bedanken voor de prettige samenwerking.

Een groot woord van dank aan alle destijds zwangere vrouwen die vrijwillig en belangeloos hebben deelgenomen aan dit onderzoek en veel vragenlijsten voor ons hebben ingevuld. Vrouwen uit alle windstreken, van Groningen tot Maastricht, waren bereid om dit allemaal te ondergaan. Zonder hun trouwe participatie was deze studie niet geslaagd.

Ik wil graag alle ex-collega's van KEMTA en ook de leden van de MTA-groep in Maastricht bedanken voor het altijd kunnen stellen van vragen, het delen van jullie fantastische kennis en voor de gezellige en inspirerende werkomgeving. Zonder jullie waren de afgelopen jaren niet zo leuk en leerzaam geweest. Beste Thea, heel erg bedankt voor je hulp bij bootstrappen in Excel. Ik heb veel geleerd van jouw geduldige uitleg, input en inspiratie.

In het bijzonder bedank ik mijn kamergenootjes Iris, Mirjam, Annemieke, Sanne en Bram. Het is erg fijn geweest om jullie in de buurt te hebben als klankbord en lotgenoot. Bedankt voor jullie waardevolle adviezen en oprechte interesse. Ik hoop dat we nog lang contact zullen houden.

Dan wil ik ook nog graag iedereen bedanken die mij op minder directe wijze heeft gesteund door er gewoon te zijn. 
Vrienden en familie, veel dank voor jullie belangstelling in mijn werk en dit proefschrift.

Mijn schoonfamilie, lieve Cor, Wilma en Monique, bedankt dat jullie altijd zo veel interesse hebben getoond en bedankt voor jullie warmte en hartelijkheid.

Lieve pap en mam, bedankt dat jullie er altijd voor me zijn. Ondanks dat het vaak moeilijk uit te leggen was wat ik nu precies deed, weet ik dat jullie trots op me zijn en onvoorwaardelijk vertrouwen in me hebben. Lieve Eline, je grote zus gaat vandaag promoveren, dat had je vast niet verwacht! Ik voel me gezegend met zo'n lieve zus, die altijd voor me klaarstaat. René, bedankt voor de belangstelling die je altijd hebt getoond in mijn werk.

Mijn grote liefde Tom, jij bent de allerbelangrijkste persoon in mijn leven. Ongelooflijk wat jij allemaal voor me doet. Zonder jouw liefde en steun was het vast niet gelukt. Het is heerlijk om alles met je te kunnen delen. Met jou aan mijn zijde voel ik mij de gelukkigste, sterkste en mooiste vrouw op aarde! Ik kijk er erg naar uit om na mijn promotie meer tijd met je door te brengen. Ik hoop dat we samen al onze dromen waar kunnen maken en dat we een lang en gezond leven tegemoet gaan. Bedankt voor alles! 

Curriculum vitae 
Denise van de Venne-Delahaije was born on March $16^{\text {th }}, 1984$ in Heerlen, the Netherlands. She graduated from secondary school (VWO) in 2002 at the Sintermeertencollege in Heerlen. From 2002 to 2007, she studied Health Sciences at Maastricht University, where she graduated within the specialization Health Policy, Economics and Management. Subsequently, she worked as a DBC project worker at the Maastricht University Medical Center. In 2008, she applied for the ZonMw funded project 'Costeffectiveness of recurrence risk guided care of pregnant women with preeclampsia in the previous pregnancy' at Maastricht University Medical Centre, department Clinical Epidemiology and Medical Technology Assessment (KEMTA). In 2014, she completed her PhD thesis. The results of her PhD project are described in this dissertation. Currently, Denise is working as data analyst at CZ.

Denise van de Venne-Delahaije is geboren op 16 maart 1984 te Heerlen. In 2002 heeft ze haar VWO diploma gehaald aan het Sintermeertencollege te Heerlen. Van 2002 tot 2007 heeft ze Gezondheidswetenschappen gestudeerd aan de Universiteit Maastricht, waar ze is afgestudeerd binnen de specialisatie Beleid en Management. Na haar studie ging ze aan de slag als DBC projectmedewerker bij het Academisch Ziekenhuis Maastricht. In 2008 is ze aan het door ZonMw gesubsidieerde onderzoek 'Kosteneffectiviteit van recidiefkans-geleide zorg bij zwangere vrouwen met preëclampsie in hun vorige zwangerschap' begonnen in het Academisch Ziekenhuis Maastricht, binnen de afdeling Klinische Epidemiologie en Medical Technology Assessment (KEMTA). In 2014 heeft ze haar promotieonderzoek afgerond. De resultaten hiervan staan beschreven in dit proefschrift. Op dit moment werkt Denise als data-analist bij CZ. 
